\title{
Anomalously Large \\ SeCond Harmonic Generation in \\ Poled Silica Glass Multilayers
}

by

Ksenia Yadav

B. A. Sc. (Waterloo) 2004

M. A. Sc. (Carleton) 2006

A thesis submitted to the Faculty of Graduate and Postdoctoral Affairs in partial fulfillment of the requirements for the degree of

Doctor of Philosophy

in

Electrical and Computer Engineering

Ottawa-Carleton Institute for Electrical and Computer Engineering

Department of Electronics

Carleton University

Ottawa, Canada

December 2011

(C) 2011

Ksenia Yadav 
Library and Archives

Canada

Published Heritage

Branch

395 Wellington Street

Ottawa ON K1A ON4

Canada
Bibliothèque et

Archives Canada

Direction du

Patrimoine de l'édition

395 , rue Wellington

Ottawa ON K1A ON4

Canada
Your file Votre référence

ISBN: 978-0-494-87760-9

Our file Notre référence

ISBN: $978-0-494-87760-9$

\section{NOTICE:}

The author has granted a nonexclusive license allowing Library and Archives Canada to reproduce, publish, archive, preserve, conserve, communicate to the public by telecommunication or on the Internet, loan, distrbute and sell theses worldwide, for commercial or noncommercial purposes, in microform, paper, electronic and/or any other formats.

The author retains copyright ownership and moral rights in this thesis. Neither the thesis nor substantial extracts from it may be printed or otherwise reproduced without the author's permission.
AVIS:

L'auteur a accordé une licence non exclusive permettant à la Bibliothèque et Archives Canada de reproduire, publier, archiver, sauvegarder, conserver, transmettre au public par télécommunication ou par l'Internet, prêter, distribuer et vendre des thèses partout dans le monde, à des fins commerciales ou autres, sur support microforme, papier, électronique et/ou autres formats.

L'auteur conserve la propriété du droit d'auteur et des droits moraux qui protege cette thèse. $\mathrm{Ni}$ la thèse ni des extraits substantiels de celle-ci ne doivent être imprimés ou autrement reproduits sans son autorisation.
In compliance with the Canadian Privacy Act some supporting forms may have been removed from this thesis.

While these forms may be included in the document page count, their removal does not represent any loss of content from the thesis.
Conformément à la loi canadienne sur la protection de la vie privée, quelques formulaires secondaires ont été enlevés de cette thèse.

Bien que ces formulaires aient inclus dans la pagination, il n'y aura aucun contenu manquant. 


\section{Abstract}

Poling techniques can be used to break the intrinsic inversion symmetry of glasses, therefore realizing materials with second-order nonlinear properties. To date, the nonlinear interactions in devices based on poled glasses have remained too weak for practical applications because of widely accepted fundamental material and process limitations. This thesis develops an approach that overcomes these limitations and demonstrates artificial silica glass structures that mimic nonlinear crystals and provide a versatile, inexpensive and widely available alternative to natural nonlinear crystals. The approach involves sub-dividing the poled glass into a multitude of glass layers deposited by standard microfabrication techniques. The experimental investigations show that doping of glass layers can be used as an effective tool to control the migration of charges that are responsible for the induced nonlinearity during the poling process. Multilayered silica structures with phosphorus, boron and germanium doping are investigated. A 14-fold improvement is achieved in the second harmonic generated (SHG) in a corona poled multilayered structure with a $2.4 \mu \mathrm{m}$-thick stack of phosphorus-doped and undoped silica layers compared to bulk silica glass poled under identical conditions. Furthermore, more than two orders of magnitude enhancement in the SHG is obtained in a thermally poled structure with a $3 \mu \mathrm{m}$-thick multilayered stack consisting of sub-100 nm-thick alternating germanium-doped and undoped silica layers. These anomalously large SHG results give a strong indication that the multilayered approach is the key to overcoming the existing challenges of poled glass devices. The high degree of control over the poling-induced nonlinearity that is offered by the multilayered design may therefore lead to practical implementations of efficient active devices in silica glass, including monolithic integration of second-order nonlinear functions directly within silica-based optical chips, and the realization of second-order nonlinear fibers. 


\section{Acknowledgements}

I would like to express my sincere gratitude to my supervisor, Professor Jacques Albert. To say that his crystal-clear scientific insights, strong values, unwavering discipline and earnest thoughtfulness were admired and appreciated would be a major understatement. His example inspired me to improve myself as a scientist and as a person, and will continue to do so in the future.

At various stages of this project, I was fortunate to discuss my work and receive advice from some of the leading experts in the area of glass poling. Many thanks go to Dr. Alexandre Kudlinski, Professor Nicolas Godbout, Professor Suzanne Lacroix, Professor Steve Brueck and Professor Yves Quiquempois for their advice and useful discussions. I am also thankful to Dr. Honglin An and Professor Simon Fleming for poling and characterizing our early samples. A special thank you goes to Professor Walter Margulis, whose in-depth insights into the topic of poled glasses and encouragement have been of great help at a key point during this project.

This research would not have been possible without the collaboration with the Communications Research Centre. A big thank you to Dr. Claire Callender, Dr. Chris Smelser, Sarkis Jacob and Dr. Chantal Blanchetiere for giving me access to their facilities, and for all their support over the past 1.5 years. Thank you also to Chris Ledderhof and Glendon Lovell for their help with the fabrication aspects of the project.

I am deeply thankful for all the help I received over the years at the Department of Electronics. Thank you to Rob Vandusen, Rick Adams and Angela Burns for patiently handling my countless non-standard fabrication requests. Many thanks for Blazenka Power and Anna Lee for their understanding, resourcefulness and cheerful disposition. Nagui Mikhail has been immensely useful on so many occasions, providing equipment and technical help that are very much appreciated. I owe a special mention to Professor Tom Ray - our shared enthusiasm for teaching and the out- 
doors resulted in some of the best memories of my time at Carleton. I am also very grateful for the many friendships that helped me get through the low points of this project. In particular, warm thanks to Alexander Beliaev, Yanina Shevchenko and Peter Chyurlia, with whom we traveled this road together.

Thank you to the Enablence team - especially to Dr. Matt Pearson, Dr. Serge Bidnyk and Dr. Ashok Balakrishnan - for their consideration and humor during the months when I joined Enablence and was finishing up my thesis.

And lastly, I would like to thank those who shared my everyday life as I traversed the many mazes of Ph.D. I am sincerely thankful to my parents for their support and encouragement, and for always pushing me to do my best. A wholehearted thank you to my husband, Bhupendra, who has endured a lot while I was climbing this "mountain called Ph.D.", and whose quiet wisdom kept me going at the toughest moments. And to my daughter, Anika, who has taught me to put things in perspective, and whose contagious energy and curiosity fuel my life and continue to amaze me every day. 


\section{Contents}

List of Figures $\quad$ vii

List of Tables $\quad x$

1 Introduction 1

1.1 Silica Glass - An Artificial Nonlinear Material . . . . . . . . . . 2

1.2 Thesis Organization . . . . . . . . . . . . . . 6

2 Second-Order Nonlinear Optics $\quad 8$

2.1 Introduction to Nonlinear Optics . . . . . . . . . . . 8

2.2 Second Harmonic Generation . . . . . . . . . . . . . . 12

2.2 .1 Phase Matching . . . . . . . . . . . . . 14

2.2 .2 Susceptibility Tensors . . . . . . . . . . . . . . 17

2.2 .3 Maker Fringe Measurements . . . . . . . . . . . . 20

2.3 Electro-Optic Modulation $\ldots \ldots \ldots \ldots . \ldots \ldots$

3 Inducing Second-Order Nonlinearities in Glasses 4

3.1 Poling Methods . . . . . . . . . . . . . . . . . . . . . . . . . . . . . . . . . .

3.2 Characterization Methods . . . . . . . . . . . . . 47

3.2 .1 Etching Methods . . . . . . . . . . . . . . . 47

3.2.2 Improved Maker Fringe Measurement Methods . . . . . . . . . 49

3.2 .3 SHG Microscopy . . . . . . . . . . . . . . . . . . . . . . . . . . . 52

3.2 .4 Other Methods . . . . . . . . . . . . . . 53

3.3 Glass Electro-Optic Modulators . . . . . . . . . . . . . . 55 
3.4 Poled Structures with Interfaces _ . . . . . . . . . . . . . 57

3.5 Poled Multilayered Structures - Thesis Objectives and Expectations . 58

4 Sample Fabrication and Characterization Methods $\quad 64$

4.1 Thin Film Deposition . . . . . . . . . . . . . . . . . . . . . 65

4.2 Poling Methods . . . . . . . . . . . . . . . . . . 67

4.3 Nonlinear Characterization Setup . . . . . . . . . . . . . . 70

5 Investigation of Corona Poled Multilayered Structures $\quad \mathbf{7 4}$

5.1 Corona Poled Bulk Glass . . . . . . . . . . . . . . . . . . . . 75

5.2 Corona Poled Multilayered Structures . . . . . . . . . . . . 78

5.3 Summary and Implications . . . . . . . . . . . . . . . . . . . 84

6 Investigation of Thermally Poled Multilayered Structures $\quad 86$

6.1 Thermally Poled Bulk Glass . . . . . . . . . . . . . . . . . 87

6.2 Unpoled Multilayered Samples . . . . . . . . . . . . . . . . . . 90

6.3 Thermally Poled Multilayered Structures with Thick Layers _. . . . 91

6.3.1 Effect of Dopants . . . . . . . . . . . . . . . . . 91

6.3 .2 Effect of Annealing . . . . . . . . . . . . . . . . 93

6.3.3 Evidence of Non-Uniform Frozen-In Electric Field Tilt . . . 95

6.4 Thermally Poled Multilayered Structures with Thin Layers . . . . . 102

6.5 Summary and Implications . . . . . . . . . . . . . . . . 108

$\begin{array}{lll}7 \text { Conclusion } & 110\end{array}$

7.1 Summary of Thesis Contributions . . . . . . . . . . . . 111

7.2 Recommendations for Future Work . . . . . . . . . . . . . 113

A Stress Study of PECVD Multilayered Samples 120

$\begin{array}{ll}\text { References } & 123\end{array}$ 


\section{List of Figures}

2.1 Different forms of phase matching in a three-wave mixing process. . . 14

2.2 Effects of various types of phase-matching on the second harmonic generation. . . . . . . . . . . . . . . 15

2.3 Wave interactions during a Maker fringe measurement. . . . . . . 21

2.4 Cross-sectional areas of the fundamental and second harmonic beams upon refraction. . . . . . . . . . . . . . . . . 23

2.5 Lab reference frame $x^{\prime}-y^{\prime}-z^{\prime}$ and sample reference frame $x-y-z$. . . . 25

2.6 Approximated dispersion of the $d_{31}$ nonlinear coefficient of lithium niobate as calculated using Miller wavelength scaling rule. . . . . . . . . 29

2.7 Calculated Maker fringe patterns for second harmonic generated in thick, uniformly nonlinear crystals. . . . . . . . . . . . . . . . 30

2.8 Calculated Maker fringe pattern for second harmonic generated in poled silica with nonlinear regions of different thicknesses. . . . . . . 30

2.9 Different implementations of the electro-optic phase modulator, and the corresponding cross-sections illustrating the propagating optical mode and the electric field. . . . . . . . . . . . . . . 38

3.1 Maker fringe SHG measurement schemes on poled glass. . . . . . . . 50

4.1 Poling methods used in this project. . . . . . . . . . . . 70

4.2 Maker fringe SHG measurement setup used for the nonlinear characterization of the poled samples. . . . . . . . . . . . . 72

4.3 Hydrofluoric acid localized etching setup. . . . . . . . . . . . . 73

5.1 Corona poled silica glass structures. . . . . . . . . . . . . . . 76 
5.2 Experimentally measured SHG in corona poled bulk glass (sample B) as the sample is etched. . . . . . . . . . . . . . . . .

5.3 Experimentally measured SHG in corona poled multilayered structures shown in Figure 5.1. . . . . . . . . . . . . . . . . . . .

5.4 Experimentally measured SHG in corona poled bulk silica glass (sample B) and multilayered sample PUPU for different poling voltages. . . .

5.5 Experimentally measured SHG in corona poled multilayered sample PUPU as the anodic side is etched.

5.6 Experimentally measured SHG in corona poled multilayered sample PUPU as both sides of the sample are etched. . . . . . . . . . . .

5.7 Experimentally measured SHG in corona poled multilayered samples PUPU and PUPU'. . . . . . . . . . . . . . . . . . . . 84

6.1 Thermally poled structures with micrometer-scale layers. . . . . . . . 87

6.2 Experimentally measured SHG in thermally poled multilayered structures with micrometer-scale layers shown in Figure 6.1. . . . . . . .

6.3 Simulated SHG in structures with varying thicknesses of the nonlinear region. ........................... 90

6.4 Linear transmission characterization of sample PU at $800 \mathrm{~nm}$. . . . . 92

6.5 Linear transmission characterization of sample PU at $400 \mathrm{~nm}$. . . . . 94

6.6 Comparison of the experimentally measured SHG in as-deposited and annealed multilayered structures.

6.7 Spatially-invariant, symmetric Maker fringe envelope characteristic of thermally poled bulk glass samples. . . . . . . . . . . . . . . 96

6.8 Spatially-varying Maker fringe envelope characteristic of thermally poled multilayered glass samples. . . . . . . . . . . . . . . . . . . . . 98

6.9 Predicted Maker fringe envelope for a nonlinear material characterized by the $\propto$ mm crystal group rotated by $10^{\circ}$. . . . . . . . . . . . . 101

6.10 Thermally poled structures with micrometer- and sub-100 nm-scale germanium-doped layers. . . . . . . . . . . . . . . . . . . . 103

6.11 Experimentally measured SHG in thermally poled multilayered structures with germanium doped layers shown in Figure 6.10. . . . . . . 104 
6.12 Remaining SHG in thermally poled multilayered structure $M$ with a $3 \mu$ m-thick stack after multiple etching experiments. . . . . . . . . . . 104

6.13 Experimentally measured SHG in thermally poled multilayered sample $\mathrm{M}$ with total stack thicknesses ranging from 1.5 to $5 \mu \mathrm{m}$. . . . . . 105 


\section{List of Tables}

2.1 Values of $K$ factors for different nonlinear processes. . . . . . . . . . 10

2.2 Summary of the effective second-order nonlinear coefficients for poled silica, crystalline quartz and lithium niobate. . . . . . . . . . . 28

4.1 Precursor gases used for the deposition of silica-based multilayered structures by LPCVD and PECVD. . . . . . . . . . . . . 65

4.2 Summary of corona poled samples investigated in this thesis. . . . . . 66

4.3 Summary of thermally poled samples with thick layers investigated in this thesis. . . . . . . . . . . . . . . . 67

4.4 Summary of thermally poled samples with thin layers investigated in this thesis. . . . . . . . . . . . . . . . . . 68

A.1 Average film stresses for multilayered structures with thick layers, deposited on fused silica and silicon substrates. . . . . . . . . . . . . . 122

A.2 Average film stresses for multilayered structures with thin layers, deposited on fused silica and silicon substrates. . . . . . . . . . . . . . 122 


\section{Chapter 1}

\section{Introduction}

Communication has always been a key factor in the advancement of human development. Optical means of communication are some of the most ancient methods of information transmission over distances. The use of smoke and reflected sunlight (the heliograph) during the day, and the use of fire during the night were developed by the Greeks to convey messages over long distances. In modern times, the use of coded light signals by navy ships and the development of the photophone - a device that could transmit sound using a beam of light reflected off a mirror - are further examples of the early attempts to use light for long distance communication. Since the middle of the $19^{\text {th }}$ century, however, electrical telegraphy and wireless radio communication schemes made tremendous progress, and pushed the optical communication systems of that era aside.

But interest in optical communication has flourished once again with the invention of the laser, and somewhat later, with the development of optical fibers. The invention of the laser in 1960 marked the beginning of photonics, a modern form of optics - a branch of science in which photons act as the agents in the generation, transmission, interaction and detection of the optical signals. Only about a decade after the invention of the laser an efficient low-loss transmission medium - the optical fiber - was introduced, paving the way for a truly long distance communication technology. 
Since the frequency of optical signals is large (a few hundred THz), optical communication systems have a capacity to transmit large amounts of information. In today's telecommunication systems, the ability to multiplex multiple signals at different wavelengths of light and to transmit them through the same fiber allows for combined bit rates in the range of terabits per second. The use of guided wave devices to control light signals can make the system compact and efficient. Moreover, the tight confinement of light in small regions of space allows it to interact with matter, exhibiting properties like the thermo-optic, acousto-optic, electro-optic, and nonlinear optical effects. The cost effectiveness, large information capacity, and immunity from electro-magnetic interference are some of the most important advantages of optical communication that allowed the phenomenal growth in information exchange that occurred in the past two decades.

This chapter introduces silica glass as a material that plays an important role in optical communications, and provides a high-level overview of the events that lead to the research of silica glass as a nonlinear material. These events and subsequent developments are tied up to the idea that is in the core of this research project. The organization of this thesis is described in the concluding section of this chapter.

\subsection{Silica Glass - An Artificial Nonlinear Material}

One material that has found a prevalent role in optical communication systems is silica glass, an amorphous form of silicon dioxide. It has been used for optical fibers primarily because of its very low absorption at the infra-red communication wavelengths. In addition, silica glass has many other useful properties. Its amorphous structure prevents undesirable effects such as polarization rotation due to birefringence, as well as enables addition of other materials in order to change its optical or electrical properties. Its high optical damage threshold allows it to withstand high intensities and thus makes it a common material in laser systems and components. Its robustness allows it to be easily shaped and polished for various optical components, 
and standard fabrication methods are routinely used for the fabrication of a wide range of silica-based integrated optical devices. Its excellent dielectric properties, including high electrical breakdown strength, and its ability to be grown on various substrate materials without concern for lattice matching problems are properties that are often utilized in microelectronic components.

One area where silica glass is not generally used is in nonlinear optical applications. The amorphous nature of silica means that it lacks long range order, and is therefore a centrosymmetric material. As will be shown later, second-order nonlinear properties do not exist in centrosymmetric materials, thus the lowest non-zero nonlinear coefficient in silica is that of third order (which is generally a much weaker effect than the second-order nonlinearity whenever both exist). Therefore, the amorphous structure of silica prevents it from being used as an active nonlinear device.

Yet, there are a number of important nonlinear applications, where the use of silica glass would be an ideal choice. One such application is in electro-optic modulation. Electro-optic modulators impress information onto the optical beam at high speeds, allowing multi-GHz response in today's optical networks. Typically modulators are realized in expensive nonlinear crystals such as lithium niobate. But because the transmission of signals in optical networks is primarily done through silica optical fibers, the large differences between the optical and thermal properties of lithium niobate and silica cause losses and integration difficulties when these modulators are used in optical networks. Yet, if such modulators could be realized in silica, all losses and integration issues would be virtually eliminated.

Another application that would greatly benefit from second-order nonlinearity in silica is frequency doubling, a nonlinear optical process in which photons interacting with a nonlinear material are effectively combined to form new photons with twice the energy, and therefore half the wavelength. As a specific example, frequency doubling of rare-earth doped fiber lasers can offer significant advantages in displays and optical data storage. These low-cost lasers are rugged, stable and compact, but their emission wavelengths are limited to a few windows within the near-IR spectrum range. A nonlinear silica fiber can be used to frequency double these lasers by direct splicing with the source, creating rugged, stable and low-cost lasers in the visible frequency 
range. In addition, the high optical damage threshold of silica also allows silica fibers with second-order nonlinear properties to be the ideal candidate for frequency doubling of high power pulsed lasers.

Another interesting application that can benefit from a nonlinear silica fiber is quantum cryptography. The main task of cryptography is to enable two parties to exchange confidential messages in such a way that the transmitted data is illegible to an eavesdropper. Usually this is done by encrypting messages between two users using a secret key that is known only to the authorized parties. Thus the security of the communication primarily depends on the security of the key distribution process. In conventional cryptography the key is distributed using schemes that are not secure, but are sufficiently difficult from the mathematical point of view to discourage an eavesdropper from performing the attack. However, although difficult, given enough resources, it is always possible for an eavesdropper to obtain the key and decrypt the transmitted data without being detected. An interesting way of closing this loophole is to use quantum cryptography, which employs quantum mechanics to achieve secure key distribution. Optical quantum cryptography relies on the quantum mechanics principles that one cannot read a quantum state without altering it, and that it is impossible to clone an unknown state [1]. Thus the process of sending the key is done using single photons, which cannot be accessed by an eavesdropper without the attack being detected. One of the practical implementations of a single photon source for this purpose relies on generating entangled photon pairs using spontaneous parametric down-conversion in a second-order nonlinear crystal. But the mismatch between the crystal source and the optical fiber causes large coupling losses and severely degrades the photon-pair rate coupled into the fiber [2]. Here the integration of a silica fiber as the nonlinear medium into the communication channel presents a big advantage as it can be used to realize an all-fiber monolithic single-photon source [3].

As these applications illustrate, there are a number of reasons to try and modify the structure of silica glass such that it does exhibit second-order nonlinear effects. Although this was not known at the time, a report in 1986 did exactly that, and as a result triggered an entire research area whose main goal was to induce secondorder nonlinearities in glasses. This report described efficient frequency doubling 
in a silica-based optical fiber by illuminating the fiber with a high-intensity pulsed beam [4]. This discovery aroused great interest because it demonstrated that secondorder nonlinear susceptibility can be created within silica glass, allowing it to be used as an active device in optical networks that are already primarily made of silica glass, thus avoiding the many integration problems that are prevalent in today's multi-material systems. This process was later termed optical poling. The observed nonlinearity was not large enough to be used in practical devices, but it motivated the search for other ways of inducing nonlinearity in glasses.

The next important milestone occurred in 1991 when a large second-order nonlinearity was induced in bulk silica [5]. This was accomplished by a simple procedure, termed thermal poling, which involved applying high voltage across the glass sample at an elevated temperature. The value of the induced second-order susceptibility this time around was approaching practical values, although the nonlinearity was not throughout the entire sample, but only in a thin region near the surface of the sample.

A great amount of work on understanding this phenomenon and enhancing its effect followed. Experiments using alternative poling methods included corona poling [6], $\mathrm{CO}_{2}$ laser-assisted poling [7], UV poling [8], UV-fs poling [9], electron-beam poling [10], and proton implantation poling [11], all successful in inducing secondorder nonlinearity of comparable magnitude to thermal poling. Work on modeling the nonlinearity formation process resulted in two models that may account for the observed nonlinearity [12]. In the first model, an effective second-order nonlinearity is created due to a permanent frozen-in electric field, which is formed due to migration of charge carriers during poling. In the second model, the creation of the second order nonlinearity is attributed to the orientation of hyper-polarizable entities.

After about eight years of intensive research, the interest in this area gradually declined. One reason for this is that no one has been able to significantly improve upon the magnitude of the second-order nonlinear coefficient that was realized in the original experiment (even the most successful experiments were able to achieve only $1.6 \mathrm{pm} / \mathrm{V}[13]$ vs. $1 \mathrm{pm} / \mathrm{V}$ achieved by [5], compared to $\sim 81 \mathrm{pm} / \mathrm{V}$ for lithium niobate). However, this by itself is not a major concern, since poled silica offers the possibility very long interaction lengths (compared to only a few centimeters 
in crystals). A much more important problem is that the induced nonlinear region extends over only a few micrometers, resulting in a poor overlap between the optical signal that propagates through the structure and this nonlinear region. As a result, all the second-order nonlinear processes remain inefficient, thus requiring unrealistically long device lengths.

The approach taken in this research project focuses on the investigation of $\mathrm{mul}$ tilayered silica-based structures as a way to increase the effective second-order nonlinearity that can be induced in silica glass. The results of a few key projects suggest that interfaces between different glass materials may be the key to enhancing the induced nonlinearity [14] [15] [16]. These projects focused on creating planar silica waveguides with at most three layers in their structures, and the most common observation was that when the layered structures were poled the nonlinearity peaked at or near material interfaces.

In the research described here, the idea of layers is taken further to create a large silica-based multilayered structure that can be used to enhance the effective second-order nonlinearity that is induced by poling. The strength of this idea lies in the realization that even if the nonlinear coefficient of each layer of silica glass is limited to $\sim 1 \mathrm{pm} / \mathrm{V}$ by fundamental physical constraints, if we create a nonlinear area that is large enough for a significant overlap between the optical signal and the nonlinear region, both electro-optic modulation and frequency doubling processes will become more efficient. Another important strength of this idea is that the required structures do not require any unusual fabrication techniques or exotic materials. The multilayered structures can be realized in a straightforward way by using well-known microfabrication techniques and standard equipment.

\subsection{Thesis Organization}

This thesis is organized in the following manner. Chapter 2 provides an introduction to nonlinear optics and the second-order nonlinear phenomena that are important for poled glass applications. Chapter 3 includes a detailed review of the progress made by other research groups on inducing second-order nonlinearities in glasses, including 
characterization methods and the modeling of the induced nonlinearity. Particular emphasis is given to reports of poled glass structures with interfaces, which motivated the original idea for our work on multilayered structures. The main idea behind this doctoral research project is described next, along with its underlying hypotheses and the practical applications that could benefit from its findings. Chapter 4 provides details on the fabrication of the multilayered structures, as well as the methods used to characterize them and the poling-induced nonlinearity.

An experimental demonstration of the second-order nonlinearity enhancement using multilayered design is the topic of the following two chapters. Chapter 5 describes corona poling experiments and findings, while Chapter 6 focuses on the thermal poling results. The last chapter includes a summary of the thesis findings, and provides recommendations for future work in the area of poled glass multilayers. 


\section{Chapter 2}

\section{Second-Order Nonlinear Optics}

The work described in this thesis focuses on the characterization, modeling and practical applications of poled silica - an artificially created, nonlinear optical material. In order to provide sufficient theoretical background for the rest of the thesis, this chapter begins with a brief introduction to nonlinear optics. Following that, second harmonic generation and the electro-optic effect - the two nonlinear phenomena that are often used for characterization of the induced nonlinearity in silica - are described.

\subsection{Introduction to Nonlinear Optics}

Optics describes the interaction of light with matter. Until relatively recently, it was thought that all optical media were linear. This implies that the optical properties of materials, such as refractive index and absorption coefficient, are independent of light intensity, that two beams of light in the same region do not interact with each other, and the frequency of light does not change by the light passing through a medium. The invention of the laser, however, enabled us to examine the behavior of light in optical materials at very high intensities. Such high intensity experiments revealed that the refractive index (and consequently the speed of light in a medium) does depend on light intensity, that the frequency of light can change as it passes 
through some media, and that photons do interact with each other. These behaviors are properties of nonlinear optics. The term nonlinear comes from the fact that the medium does not respond linearly to the strength of the optical wave.

All dielectric materials consist of atoms with a nucleus of positive charge, surrounded by negatively charged electrons. In the presence of an applied electric field $\mathbf{E}(t)$, these positive and negative charges are displaced in opposite directions. This distorts (or polarizes) the material, and as a result the electric field induces a dipole moment within the medium. This distortion effect is usually characterized by the polarization $\mathbf{P}(t)$, which is defined as the dipole moment per unit volume. The properties of a dielectric medium through which an optical wave propagates are described by the following relation between the induced polarization and the applied electric field:

$$
\mathbf{P}(t)=\epsilon_{0}[\underbrace{\chi^{(1)} \mathbf{E}(t)}_{\text {linear }}+\underbrace{\chi^{(2)} \mathbf{E}^{2}(t)+\chi^{(3)} \mathbf{E}^{3}(t)+\ldots+\chi^{(n)} \mathbf{E}^{n}(t)}_{\text {nonlinear }}]
$$

where $\epsilon_{0}$ is the permittivity of free space, $\chi^{(1)}$ is the linear susceptibility of the medium, $\chi^{(2)}$ is the second-order susceptibility, and in general $\chi^{(n)}$ is the $(n+1)^{\text {th }}$ rank susceptibility tensor relating the electric field to the nonlinear polarization. As can be guessed from equation (2.1), a linear dielectric medium is characterized by a linear relation between the induced polarization and the electric field, so the above reduces to $\mathbf{P}(t)=\epsilon_{0} \chi^{(1)} \mathbf{E}(t)$. Since the nonlinear susceptibility coefficients are typically small (order-of-magnitude values are $\chi^{(1)} \sim 1, \chi^{(2)} \sim 10^{-11} \mathrm{~m} / \mathrm{V}$ and $\chi^{(3)} \sim 10^{-21}$ $\left.(\mathrm{m} / \mathrm{V})^{2}[17]\right)$, the nonlinear polarization effects can only be observed if very large field intensities are used.

While equation (2.1) describes the time domain polarization response, it is more common to use the frequency domain response. In this case, the Fourier components of the nonlinear polarization take the form:

$P_{i}^{(n)}\left(\omega_{n+1}\right)=\epsilon_{0} \sum_{j k \ldots m} K\left(-\omega_{n+1} ; \omega_{1}, \ldots, \omega_{n}\right) \chi_{i j k \ldots m}^{(n)}\left(-\omega_{n+1} ; \omega_{1}, \ldots, \omega_{n}\right) E_{j}\left(\omega_{1}\right) E_{k}\left(\omega_{2}\right) \ldots E_{m}\left(\omega_{n}\right)$

where $P_{i}^{(n)}$ is the $i^{\text {th }}$ component of the $n^{\text {th }}$ order polarization field, $E_{i}$ the $i^{\text {th }}$ com- 
ponent of the electric field amplitude, and $\chi_{i j k \ldots m}^{(n)}$ is the $n^{\text {th }}$ order susceptibility tensor (a tensor of rank $(n+1)$ ). The indices $i, j, k, \ldots, m$ each take the values of the cartesian coordinates $x, y$ and $z$, and the conservation of energy requires that $\omega_{n+1}=\omega_{1}+\omega_{2}+\ldots+\omega_{n} . K$ is a numerical factor that is defined as [18]:

$$
K\left(-\omega_{n+1} ; \omega_{1}, \ldots, \omega_{n}\right)=2^{l+m-n} p
$$

where $p$ is the number of distinct permutations of $\omega_{1}, \ldots, \omega_{n}, n$ is the order of the nonlinearity, $m$ is the number of DC fields present, and $l=1$ if $\omega_{n+1} \neq 0$ or otherwise $l=0$. Table 2.1 contains the values of the $K$ factor for the nonlinear processes that are of interest to this thesis.

\begin{tabular}{lccc}
\hline Nonlinear Process & ORdER OF NONLINEARITY & FrequenCIES & $K$ \\
\hline Linear electro-optic effect & 2 & $-\omega ; 0, \omega$ & 2 \\
Second harmonic generation & 2 & $-2 \omega ; \omega, \omega$ & $\frac{1}{2}$ \\
\hline
\end{tabular}

Table 2.1: Values of $K$ factors for different nonlinear processes.

For a specific case of e.g. a second-order polarization oscillating at $\omega_{3}$ due to the presence of fields oscillating at frequencies $\omega_{1}$ and $\omega_{2}$ (with $\omega_{3}=\omega_{1}+\omega_{2}$ ), equation $(2.2)$ reduces to:

$$
P_{i}^{(2)}\left(\omega_{3}\right)=\epsilon_{0} \sum_{j k} K\left(-\omega_{3} ; \omega_{1}, \omega_{2}\right) \chi_{i j k}^{(2)}\left(-\omega_{3} ; \omega_{1}, \omega_{2}\right) E_{j}\left(\omega_{1}\right) E_{k}\left(\omega_{2}\right)
$$

where $\chi_{i j k}^{(2)}$ is the second-order complex susceptibility and the Fourier transform of $\chi^{(2)}(t)$.

From equation (2.4) it can be concluded that only non-centrosymmetric materials can give rise to a second-order (or any even-order) nonlinear response. This is based on Neumann's principle, which states that, if a crystal is invariant with respect to certain symmetry elements, any of its physical properties must also be invariant with respect to the same symmetry elements [19]. A system is centrosymmetric when its physical properties remain unchanged under the inversion symmetry transformation (i.e. $x \rightarrow-x, y \rightarrow-y, z \rightarrow-z$ ). This means that a reversal of signs of $E_{j}\left(\omega_{1}\right)$ and 
$E_{k}\left(\omega_{2}\right)$ in equation (2.4) must also cause a reversal in the sign of $P_{i}^{(2)}\left(\omega_{3}\right)$. Thus the following must be true:

$$
-\epsilon_{0} \sum_{j k} \chi_{i j k}^{(2)}\left(-\omega_{3} ; \omega_{1}, \omega_{2}\right) E_{j}\left(\omega_{1}\right) E_{k}\left(\omega_{2}\right)=\epsilon_{0} \sum_{j k} \chi_{i j k}^{(2)}\left(-\omega_{3} ; \omega_{1}, \omega_{2}\right)\left[-E_{j}\left(\omega_{1}\right)\right]\left[-E_{k}\left(\omega_{2}\right)\right]
$$

which can only be satisfied if $\chi_{i j k}^{(2)}=0$. This means that no even-order response (such as second harmonic generation) can come from a centrosymmetric medium. A similar argument can also be used to show that all materials can display third-order nonlinearities.

Because the study of nonlinear optics has been approached from many disciplines, a few different conventions and notations have been developed to describe nonlinear optical phenomena. The conventions differ in the way they define the nonlinear polarization and the interacting fields in the frequency domain. A review of the different conventions and how they relate to each other can be found in [20]. Another source of confusion is the units used to report nonlinear optical values. A summary of the fundamental quantities of nonlinear optics, as well as their corresponding values in different unit systems and conversion factors are given in [19] and in [21]. In this thesis, the convention as described by equation (2.2) and the SI system of units will be used.

Despite the fact that the nonlinear optical interactions cause only small perturbations, the corresponding nonlinear optical phenomena are used in a wide variety of applications. The second-order (quadratic) nonlinear relation between $\mathbf{P}$ and $\mathbf{E}$ gives rise to a variety of three-wave mixing phenomena, where three monochromatic waves interact according to laws of energy and momentum conservation. One application of three-wave mixing is frequency conversion - two monochromatic waves mix to generate a third wave at their sum or difference frequencies. An important special case of this is second harmonic generation - essentially frequency doubling (or halving the wavelength) of a monochromatic wave. Two monochromatic waves can also be used to amplify a third wave, a process known as parametric amplification, which, with an incorporation of a feedback, can create an oscillator. Another important appli- 
cation that is directly related to second-order nonlinearity is the linear electro-optic (or Pockels) effect, which describes a linear dependency of the refractive index of a medium on the applied electric field.

The third-order (cubic) relation between $\mathbf{P}$ and $\mathbf{E}$ gives rise to four-wave mixing phenomena, including third-harmonic generation, self-phase modulation and selffocusing. A related effect is the quadratic electro-optic (or Kerr) effect, in which the refractive index changes in proportion to the square of the applied electric field.

In the context of a study of poled silica glass, second harmonic generation and the linear electro-optic effect are particularly important for characterization of the induced nonlinearity. These two nonlinear phenomena are described in more detail in the next two sections.

\subsection{Second Harmonic Generation}

Three-wave mixing is a process where three waves at frequencies $\omega_{1}, \omega_{2}$ and $\omega_{3}=\omega_{1}+\omega_{2}$ interact. During this process, two photons of lower frequency are annihilated, and a photon of higher frequency is created. Since $\hbar \omega$ and $\hbar \mathbf{k}$ are the energy and momentum of a photon of frequency $\omega$ and wavevector $\mathbf{k}$, conservation of energy and momentum requires that:

$$
\begin{aligned}
& \hbar \omega_{1}+\hbar \omega_{2}=\hbar \omega_{3} \\
& \hbar \mathbf{k}_{1}+\hbar \mathbf{k}_{2}=\hbar \mathbf{k}_{3}
\end{aligned}
$$

which are known as the frequency and the phase matching conditions.

Second harmonic generation (SHG) is a special case of three-wave mixing, where the interaction of two waves with frequencies $\omega$ and $2 \omega$ is considered. In this case, two photons of the fundamental wave (of frequency $\omega$ ) are converted to a single photon of the second harmonic (SH) wave, whose frequency is $2 \omega$. Based on the nonlinear wave equation it can be shown that the growth of the second harmonic field as a function of propagation distance in a nonlinear material is given by [22]:

$$
E_{2 \omega}(z)=\epsilon_{0} \frac{-j \omega}{2} \sqrt{\frac{\mu_{0}}{\epsilon}} E_{\omega}^{2} \int_{0}^{z} \chi_{e f f} e^{j \Delta k z} d z
$$


where $E_{\omega}$ and $E_{2 \omega}$ are the fundamental and second harmonic complex field amplitudes respectively, $\omega$ is the frequency of the fundamental wave, $\mu_{0}$ is the permeability of free space, $\epsilon$ is the permittivity of the material, $z$ is the propagation distance in the nonlinear material, $\Delta k$ is the wavenumber mismatch term (which will be explained in detail in Section 2.2.1), and $\chi_{\text {eff }}$ is the effective nonlinear coefficient - a scalar quantity that relates the nonlinear polarization and the electric field, and is determined based on a given symmetry and electric field polarization. This equation is valid when the electric field $E_{\omega}$ does not vary significantly over the optical wavelength (the slowly varying envelope approximation) and when the amount of power lost from the fundamental beam by conversion to the second harmonic is negligible (the non-depletion approximation).

Equation (2.8) can be used to calculate the second harmonic field for a given geometry and nonlinearity profile. For example, the SH generated in a slab of thickness $L$, which has a uniform nonlinearity profile, is:

$$
E_{2 \omega}(z)=\epsilon_{0} \frac{-j \omega}{2} \sqrt{\frac{\mu_{0}}{\epsilon}} E_{\omega}^{2} \chi_{e f f} \frac{e^{j \Delta k L}-1}{j \Delta k}
$$

In a typical SHG experiment, the measured signal is the power of the second harmonic light. The intensity of a monochromatic wave is given by [17]:

$$
I=\frac{|E|^{2}}{2 \eta}
$$

where $\eta$ is the impedance of the medium that can be expressed as:

$$
\eta=\sqrt{\frac{\mu_{0}}{\epsilon}}=\frac{1}{n_{2 \omega} \epsilon_{0} c}
$$

where $n_{2 \omega}$ is the refractive index of the medium at the $\mathrm{SH}$ wavelength, $c$ is the speed of light in vacuum, and the relationship $\epsilon=\epsilon_{0} n^{2}$ was used. Using the above three equations, the second harmonic intensity can be written as:

$$
I_{2 \omega}=\epsilon_{0}^{2} \frac{\omega^{2} L^{2}}{2 n_{\omega}^{2} n_{2 \omega}}\left(\frac{\mu_{0}}{\epsilon_{0}}\right)^{\frac{3}{2}} \chi_{e f f}^{2} I_{\omega}^{2}\left[\frac{\sin \left(\frac{\Delta k L}{2}\right)}{\frac{\Delta k L}{2}}\right]^{2}
$$

which can alternatively be expressed as:

$$
I_{2 \omega}=\frac{\omega^{2} L^{2}}{2 \epsilon_{0} c^{3} n_{\omega}^{2} n_{2 \omega}} \chi_{e f f}^{2} I_{\omega}^{2}\left[\frac{\sin \left(\frac{\Delta k L}{2}\right)}{\frac{\Delta k L}{2}}\right]^{2}
$$


This equation is very important for the experimental part of this work. It contains a number of straightforward parameters, including the refractive index of the material at the fundamental wavelength $n_{\omega}$, and others that have been previously defined. There are, however, two parameters that require some more explanation. The first is $\Delta k$, the wavenumber mismatch term - this term plays a crucial role in the efficiency of the SHG process, and it is described in detail in Section 2.2.1. The second important parameter is $\chi_{e f f}$, the effective second-order nonlinear coefficient. The dependence of $\chi_{e f f}$ on the symmetry of a nonlinear material is described in Section 2.2.2, and its actual form, as determined by the material geometry and the experimental measurement setup, is demonstrated in Section 2.2.3.

\subsubsection{Phase Matching}

The second harmonic intensity, described by equation (2.12), includes a term that depends on the wavenumber mismatch term $\Delta k$. This is an important factor that determines the efficiency of the second harmonic generation process.

The simplest case of a three-wave mixing process is when the three waves are collinear and the medium is non-dispersive (i.e. the fundamental and the $\mathrm{SH}$ waves propagate with the same phase velocity along the entire length of the nonlinear material), as shown in Figure 2.1(a). In this case, the power flows continuously from the fundamental wave to the second harmonic, so in principle all the fundamental power can be converted into SH power [23], as shown in Figure 2.2(a). This ideal condition is called perfect phase matching, i.e. $\Delta \mathbf{k}=\mathbf{k}_{\mathbf{3}}-\mathbf{k}_{\mathbf{2}}-\mathbf{k}_{\mathbf{1}}=\mathbf{0}$.

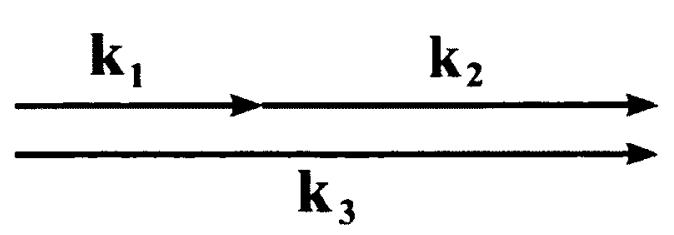

(a)

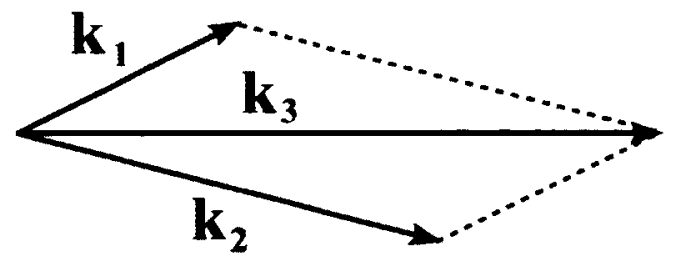

(b)

Figure 2.1: Different forms of phase matching in a three-wave mixing process: (a) collinear phase matching, (b) vector phase matching. 


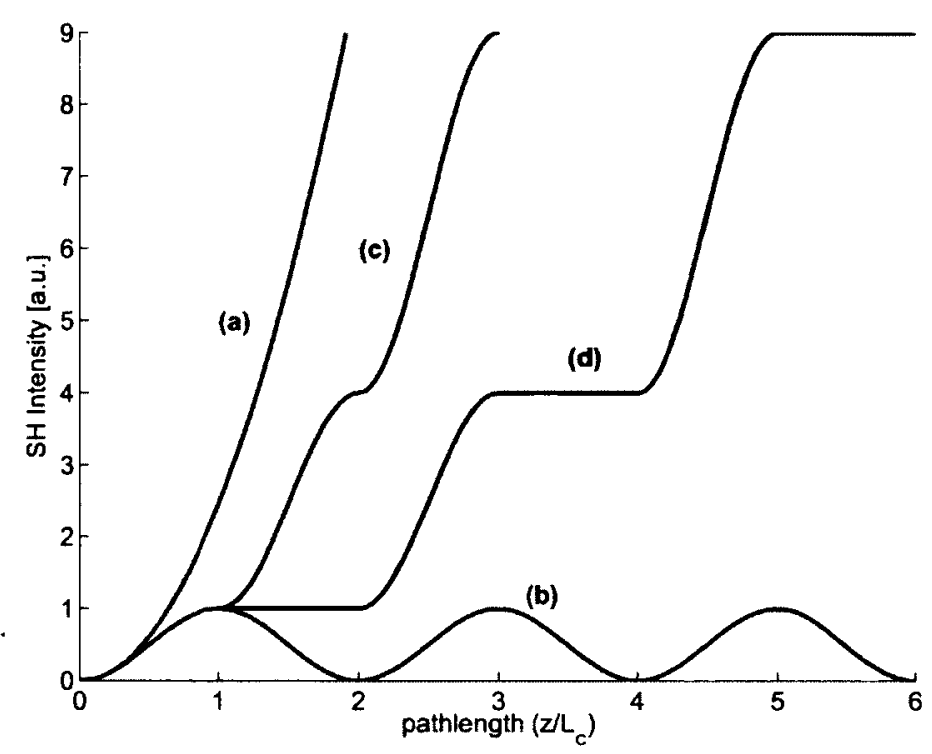

Figure 2.2: Effects of various types of phase-matching on the second harmonic generation: (a) perfect phase matching, (b) no phase matching, (c) quasi-phase matching (reversal), (d) quasi-phase matching (erasure).

However, all materials are in reality dispersive, thus the three waves actually travel at different phase velocities. This is the non-perfect phase matching case, when $\Delta \mathbf{k}=\mathbf{k}_{\mathbf{3}}-\mathbf{k}_{\mathbf{2}}-\mathbf{k}_{\mathbf{1}} \neq 0$. According to equation (2.12), when the phase-matching term $\Delta k$ is non-zero, a periodic conversion of the fundamental field into the SH field as a function of distance is expected. Because the fundamental and the $\mathrm{SH}$ waves do not travel at the same velocities, the $\mathrm{SH}$ waves generated at different locations within the nonlinear material are not always in phase - at some point, destructive interference of the $\mathrm{SH}$ waves causes a decrease in the generated $\mathrm{SH}$ and the power flows back into the fundamental wave. The periodicity of this effect is quantified by a parameter known as the coherence length, which is essentially a measure of the maximum "useful" length of the nonlinear material, and can be defined as:

$$
L_{c}=\frac{\pi}{|\Delta k|}
$$

The literature on nonlinear optics includes various definitions of this parameter. Some references (e.g. [17] or [24]) define it as the difference between the maximum point and the first minima when the $\operatorname{sinc}^{2}(\Delta k L / 2)$ term is graphed vs. $(\Delta k L / 2)$, which 
results in $L_{c}=2 \pi /|\Delta k|$. In other references (e.g. [25], [21] or [26]), it is the distance over which constructive (or destructive) interference occurs, in which case it is given by equation (2.14). Yet others (e.g. [22]) define it as $L_{c}=2 /|\Delta k|$. For the case of second harmonic generation, $\Delta k=k_{2 \omega}-2 k_{\omega}$, which leads to:

$$
\Delta k=\frac{4 \pi}{\lambda_{\omega}}\left(n_{2 \omega}-n_{\omega}\right)
$$

Using the definition given by equation (2.14) (which will be used throughout this thesis), $L_{c}$ can therefore be expressed as a function of the fundamental wavelength and the refractive indices of the two beams as:

$$
L_{c}=\frac{\lambda_{\omega}}{4\left|n_{2 \omega}-n_{\omega}\right|}
$$

When there is no phase matching (i.e. $\Delta k \neq 0$ ) no significant growth in the second harmonic signal can be achieved. As shown in Figure 2.2(b), the intensity of the SH grows for one coherence length, and then decreases to zero again due to destructive interference. However, by using vector addition of the wavevectors, as shown in Figure 2.1(b), it is possible to achieve perfect phase matching by careful design of the experiment. A traditional method to achieve such phase matching is to use birefringent nonlinear materials [27]. In a crystal for which the ordinary and the extraordinary refractive indices are different, it is possible to achieve phase matching for SHG by finding an angle of propagation for which the refractive indices for the fundamental and the $\mathrm{SH}$ waves are identical. An interesting variation of this is temperature tuning [28], where the birefringence of a crystalline material is precisely controlled using temperature, such that the phase matching condition is satisfied. However, precise phase matching using methods that rely on birefringence is difficult to achieve, and they severely constrain the choice of crystal configuration.

A more flexible way to achieve significant second harmonic growth is to use quasiphase matching (QPM), where the main idea is that the nonlinear susceptibility is modulated with a period that matches the coherence length in the nonlinear medium. One way to achieve this is to reverse the nonlinear polarization every coherence length as is done with periodically-poled lithium niobate devices [29]. This is accomplished 
by alternating the polarity of patterned electrodes, and essentially accomplishing periodic poling. Such quasi-phase matching causes the second harmonic to continuously grow, as shown in Figure 2.2(c).

Another form of quasi-phase matching is to eliminate the regions where normally destructive interference would occur. Although this results in less efficient second harmonic generation compared to the quasi-phase matching method described in the previous paragraph, this technique is more feasible in practice. In this form of quasiphase matching, only every other coherence length is nonlinear, while the intermittent regions are not. This can be accomplished by using a periodic poling electrode [30] or by periodic erasure of a uniformly induced nonlinearity [31]. The resultant growth in the second harmonic signal is shown in Figure 2.2(d).

When the interaction is phase-matched, equation (2.12) can be used to obtain a direct measurement of the nonlinear susceptibility. However, this is not an easy experiment, since the interaction length, material parameters, as well as the properties of the fundamental beam (power, and in the case of pulsed fundamental beam, the spatial and temporal distribution of the pulses) must be precisely known [32]. An easier and more common technique to measure the nonlinear susceptibility is by means of a relative measurement with a material of a known second-order nonlinearity, which will be described in detail in Section 2.2.3.

\subsubsection{Susceptibility Tensors}

An important parameter in equation (2.12) is $\chi_{\text {eff }}$, the effective second-order nonlinear coefficient, a scalar quantity that is determined based on a material symmetry and electric field polarization. This section briefly describes the general form of the second-order susceptibility tensor, and the crystal symmetry groups of a few materials that are relevant to this project.

The second-order polarization, as was previously defined in equation (2.4), reduces to the following form for second harmonic generation:

$$
P_{i}^{(2)}=\frac{\epsilon_{0}}{2} \sum_{j k} \chi_{i j k}^{(2)}(-2 \omega ; \omega, \omega) E_{j}(\omega) E_{k}(\omega)
$$


$\chi_{i j k}^{(2)}$ is a tensor of third rank, and thus in general has 27 components. When the nonlinear optical interactions of interest involve waves whose frequencies are much smaller than the lowest resonance frequency of the material system (a condition known as Kleinman's symmetry [18]), the susceptibility tensor $\chi_{i j k}$ is symmetric in its last two indices. Therefore, the notation can be simplified by reducing the $\chi_{i j k}$ tensor to a $3 \times 6 \chi_{i l}$ matrix using the following new indices:

$\begin{array}{ccccccc}j k: & 11 & 22 & 33 & 23,32 & 31,13 & 12,21 \\ l: & 1 & 2 & 3 & 4 & 5 & 6\end{array}$

Thus the nonlinear polarization leading to second harmonic generation can be described as:

$$
\left[\begin{array}{c}
P_{x}^{2 \omega} \\
P_{y}^{2 \omega} \\
P_{z}^{2 \omega}
\end{array}\right]=\frac{\epsilon_{0}}{2}\left[\begin{array}{llllll}
\chi_{11} & \chi_{12} & \chi_{13} & \chi_{14} & \chi_{15} & \chi_{16} \\
\chi_{21} & \chi_{22} & \chi_{23} & \chi_{24} & \chi_{25} & \chi_{26} \\
\chi_{31} & \chi_{32} & \chi_{33} & \chi_{34} & \chi_{35} & \chi_{36}
\end{array}\right]\left[\begin{array}{c}
E_{x}(\omega)^{2} \\
E_{y}(\omega)^{2} \\
E_{z}(\omega)^{2} \\
2 E_{y}(\omega) E_{z}(\omega) \\
2 E_{x}(\omega) E_{z}(\omega) \\
2 E_{x}(\omega) E_{y}(\omega)
\end{array}\right]
$$

Often, especially in experimental work, the $\chi_{i l}^{(2)}$ is replaced by the second-order nonlinear parameter $d_{i l}$, and the two are related through $d_{i l}=\frac{1}{2} \chi_{i l}^{(2)}$ [33]. Therefore, equation (2.18) can be rewritten as:

$$
\left[\begin{array}{c}
P_{x}^{2 \omega} \\
P_{y}^{2 \omega} \\
P_{z}^{2 \omega}
\end{array}\right]=\epsilon_{0}\left[\begin{array}{llllll}
d_{11} & d_{12} & d_{13} & d_{14} & d_{15} & d_{16} \\
d_{21} & d_{22} & d_{23} & d_{24} & d_{25} & d_{26} \\
d_{31} & d_{32} & d_{33} & d_{34} & d_{35} & d_{36}
\end{array}\right]\left[\begin{array}{c}
E_{x}(\omega)^{2} \\
E_{y}(\omega)^{2} \\
E_{z}(\omega)^{2} \\
2 E_{y}(\omega) E_{z}(\omega) \\
2 E_{x}(\omega) E_{z}(\omega) \\
2 E_{x}(\omega) E_{y}(\omega)
\end{array}\right]
$$

When the Kleinman symmetry condition is valid, $\chi_{i l}$ has only 10 independent elements. Moreover, any symmetry property of a given nonlinear optical material imposes additional restrictions on the form of the nonlinear susceptibility tensor. A detailed explanation of the different symmetry requirements is provided in [22], 
and a comprehensive summary of the different crystal classes, their corresponding symmetries and second-order susceptibility tensors can be found in [34].

In the context of this thesis, three crystalline materials will be encountered single-crystalline quartz, lithium niobate and poled silica glass. The reduced susceptibility tensors for these materials are provided below for future reference.

Crystalline quartz, a crystalline form of silicon dioxide, is one of the most abundant minerals found in nature, and its unique properties make it a useful substance in many electrical and optical applications. It is a positive uniaxial crystal that is often used as a standard reference in nonlinear optics experiments. Its crystal symmetry belongs to the 32 crystal group, which has the following second-order susceptibility tensor:

$$
\left[\begin{array}{cccccc}
\chi_{11} & -\chi_{11} & 0 & \chi_{14} & 0 & 0 \\
0 & 0 & 0 & 0 & -\chi_{14} & -\chi_{11} \\
0 & 0 & 0 & 0 & 0 & 0
\end{array}\right]
$$

Lithium niobate $\left(\mathrm{LiNbO}_{3}\right)$ is a negative uniaxial crystalline material that is often used in linear and nonlinear optical applications. Its most widespread use is in electrooptic modulators, thus any search for alternative materials for this purpose typically uses the nonlinear properties of lithium niobate as a reference. It has a $3 \mathrm{~m}$ crystal symmetry, therefore its reduced nonlinear susceptibility tensor takes the form:

$$
\left[\begin{array}{cccccc}
0 & 0 & 0 & 0 & \chi_{31} & -\chi_{22} \\
-\chi_{22} & \chi_{22} & 0 & \chi_{31} & 0 & 0 \\
\chi_{31} & \chi_{31} & \chi_{33} & 0 & 0 & 0
\end{array}\right]
$$

Silica, as an amorphous material, is centrosymmetric, and therefore does not exhibit any second-order nonlinear effects. However, the symmetry in silica may be broken by using poling techniques, which create an artificial crystalline structure within the material. Because poling is done perpendicular to the surface, the created structure has a single axis of symmetry (along the direction of the applied electric field, e.g. $z$-axis) and two degenerate axes ( $x$ and $y$ axes). This structure is characteristic of the $\infty \mathrm{mm}$ (as well as $6,6 \mathrm{~mm}, 4$ and $4 \mathrm{~mm}$ ) crystal group [35]. The reduced 
nonlinear susceptibility tensor of this group is given by:

$$
\left[\begin{array}{cccccc}
0 & 0 & 0 & 0 & \chi_{31} & 0 \\
0 & 0 & 0 & \chi_{31} & 0 & 0 \\
\chi_{31} & \chi_{31} & \chi_{33} & 0 & 0 & 0
\end{array}\right]
$$

\subsubsection{Maker Fringe Measurements}

A common way to measure the nonlinear susceptibility of a material is to compare the signal generated by the nonlinear material to the signal from a previously measured "standard" (such as crystalline quartz or lithium niobate). This method is known as a Maker fringe measurement, first suggested in [36] and later derived in detail in [25]. To obtain a measurement of the nonlinear coefficient, a laser beam is focused onto the sample, and while the sample is rotated, the second harmonic generated in the sample is measured. A simple ray diagram depicting the setup is shown in Figure 2.3(a). Due to dispersion, the fundamental and the second harmonic waves propagate at slightly different angles, $\theta_{\omega}$ and $\theta_{2 \omega}$, which are related through Snell's law as:

$$
\sin \theta=n_{\omega} \sin \theta_{\omega}=n_{2 \omega} \sin \theta_{2 \omega}
$$

The intensity of the second harmonic generated inside a bulk nonlinear sample was previously shown to be given by equation (2.13). In a Maker fringe measurement, the fundamental wave enters the sample at an angle $\theta$. If $L$ in equation (2.13) is replaced by the angle-dependent nonlinear pathlength in the sample $L / \cos \theta_{2 w}$, the calculated intensity reveals periodic variations as the incidence angle is varied. A detailed theoretical analysis reveals that these oscillations in the SH intensity occur as a result of interference between the bound and the free second harmonic waves [25], as shown in Figure 2.3(b). The bound wave, propagating with wave vector $\mathbf{k}_{b}=2 \mathbf{k}_{\omega}$, is the radiated wave at frequency $2 \omega$ that arises from the induced second-order nonlinear polarization $\mathbf{P}_{2 \omega}^{(2)}$. It is bound to the fundamental wave and propagates in the same direction (at an angle $\theta_{\omega}$ ). The free wave, also at frequency $2 \omega$, results from the second harmonic light that is already generated at the input surface and at each subsequent 


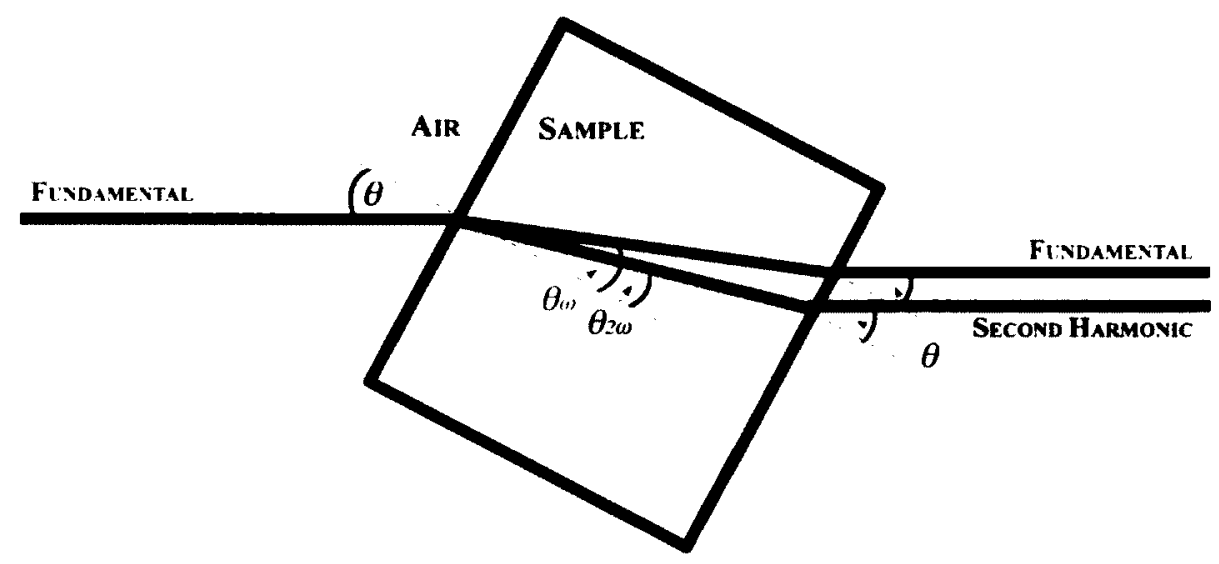

(a)

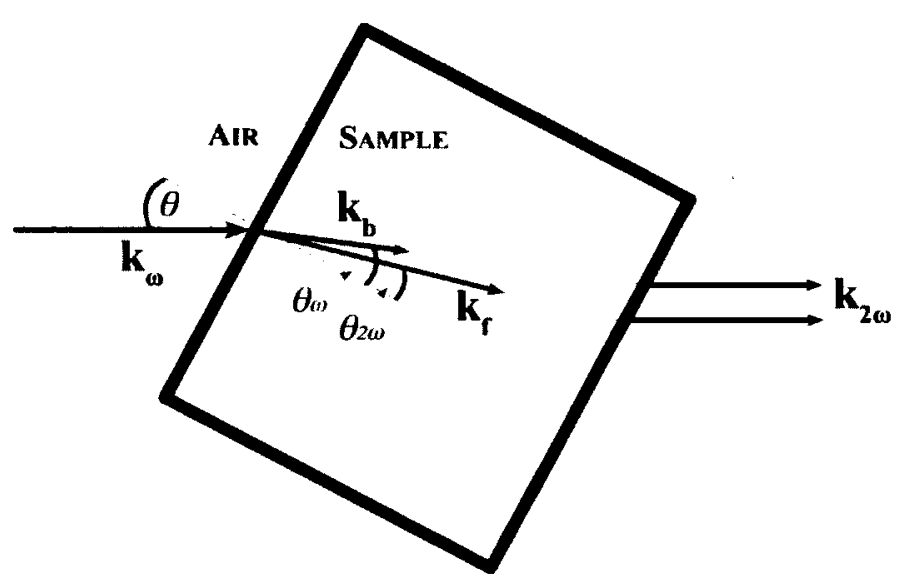

(b)

Figure 2.3: Wave interactions during a Maker fringe measurement: (a) propagation of the fundamental and the second harmonic waves, (b) wavevectors of the bound and the free second harmonic waves.

"increment" of the sample, and propagates with a wave vector $\mathbf{k}_{f}=\mathbf{k}_{2 \omega}$ (at an angle $\left.\theta_{2 \omega}\right)$. The phase difference between the free and the bound waves results in the periodic oscillations as the sample is rotated relative to the incident fundamental beam.

A simpler picture of the principle of operation of the Maker fringes measurement can be obtained if the fundamental and the second harmonic beams are approximated to be always collinear (i.e. $\theta_{\omega} \approx \theta_{2 \omega}$ ). For the specific case of silica glass this approximation is justified - due to the low dispersion of silica the maximum deviation 
between the two propagation angles is $0.6^{\circ}$, which occurs only at very high incidence angles. In addition, this approximation offers an intuitive interpretation of the Maker fringes measurement as a change in the effective path length in the nonlinear material as the sample is rotated.

In a Maker fringe experiment the fundamental and the second harmonic waves undergo refraction at the front and back faces of the sample, therefore the expression of the measured second harmonic power must include factors related to Fresnel transmissions. At the air-sample interface, the power transmittance of the fundamental beam is given by:

$$
T_{\omega}=\frac{n_{\omega} \cos \theta_{\omega}}{\cos \theta}\left|t_{a \rightarrow s}^{2}\right|
$$

where $t_{a \rightarrow s}$ is a standard Fresnel transmission term for the air-sample interface. For the two different polarizations of the signal, it is given by:

$$
\begin{aligned}
t_{a \rightarrow s}^{p-p o l} & =\frac{2 \cos \theta}{n_{\omega} \cos \theta+\cos \theta_{\omega}} \\
t_{a \rightarrow s}^{s-p o l} & =\frac{2 \cos \theta}{\cos \theta+n_{\omega} \cos \theta_{\omega}}
\end{aligned}
$$

Similarly, at the sample-air interface, the power transmittance of the second harmonic beam is:

$$
T_{2 \omega}=\frac{\cos \theta}{n_{2 \omega} \cos \theta_{2 \omega}}\left|t_{s \rightarrow a}^{2}\right|
$$

where $t_{s \rightarrow a}$ is the Fresnel transmission term for the sample-air interface, which is given for the two polarizations as:

$$
\begin{aligned}
t_{s \rightarrow a}^{p-p o l} & =\frac{2 n_{2 \omega} \cos \theta_{2 \omega}}{\cos \theta_{2 \omega}+n_{2 \omega} \cos \theta} \\
t_{s \rightarrow a}^{s-p o l} & =\frac{2 n_{2 \omega} \cos \theta_{2 \omega}}{n_{2 \omega} \cos \theta_{2 \omega}+\cos \theta}
\end{aligned}
$$

Because the measured signal in the Maker fringes SHG experiment is the power of the second harmonic, we must take into account the fact that the cross-sections of the beam outside and inside the sample are different due to refraction. This is illustrated in Figure 2.4, where $A$ denotes the cross-sectional area of the fundamental 


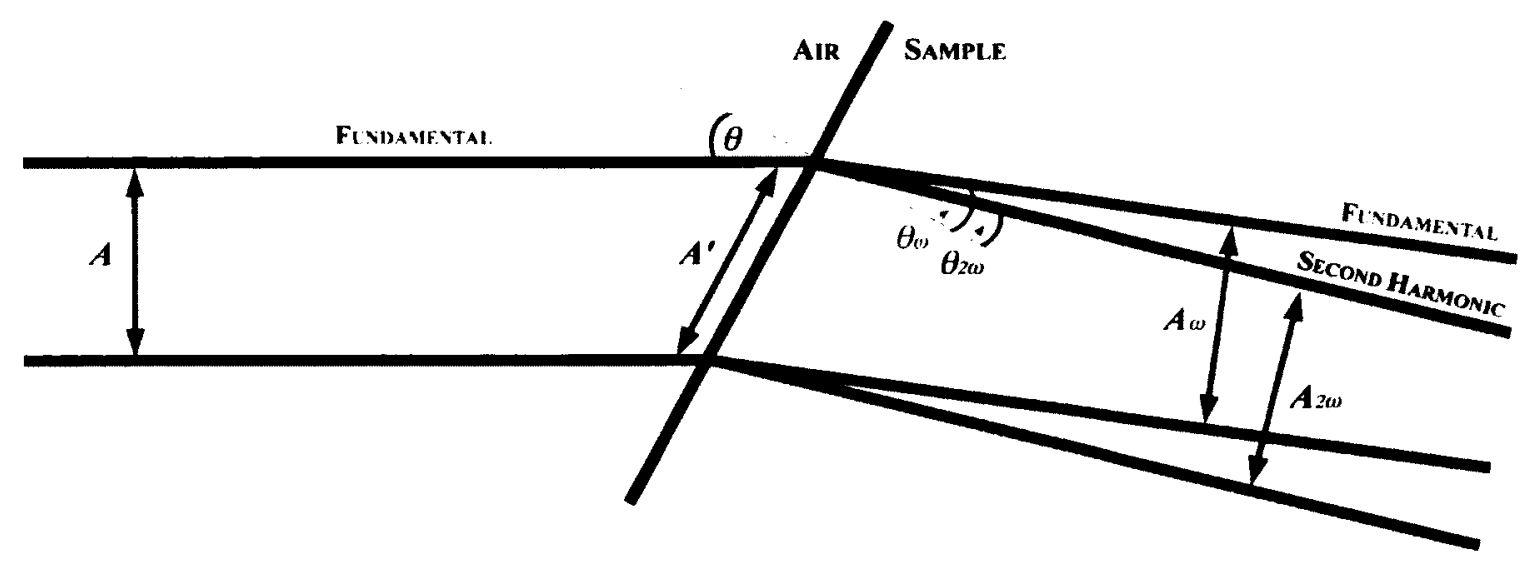

Figure 2.4: Cross-sectional areas of the fundamental and second harmonic beams upon refraction.

beam outside the sample, $A^{\prime}$ is its projection onto the rotated sample, and $A_{\omega}$ and $A_{2 \omega}$ are the cross-sections of fundamental and $\mathrm{SH}$ beams inside the sample, respectively. From geometrical considerations these parameters are related by:

$$
\begin{aligned}
& A^{\prime}=\frac{A}{\cos \theta} \\
& A_{\omega}=A^{\prime} \cos \theta_{\omega}=A \frac{\cos \theta_{\omega}}{\cos \theta} \\
& A_{2 \omega}=A^{\prime} \cos \theta_{2 \omega}=A \frac{\cos \theta_{2 \omega}}{\cos \theta}
\end{aligned}
$$

Therefore, since $I_{2 \omega} \propto I_{\omega}^{2}$, the expression for the second harmonic power must include a beam correction factor $B$, which is defined as following:

$$
\begin{aligned}
P_{2 \omega} & \propto P_{\omega}^{2} \frac{A_{2 \omega}}{A_{\omega}^{2}} \\
& =\frac{P_{\omega}^{2}}{A} \underbrace{\left(\frac{\cos \theta_{2 \omega}}{\cos \theta}\right)\left(\frac{\cos \theta}{\cos \theta_{\omega}}\right)^{2}}_{B}
\end{aligned}
$$

Finally, by combining equations (2.13) and (2.24)-(2.33), the expression for the second harmonic power measured in a Maker fringe experiment is derived to be:

$$
P_{2 \omega}=\frac{\omega^{2} L^{2}}{2 \epsilon_{0} c^{3} n_{\omega}^{2} n_{2 \omega}} B(\theta) \chi_{e f f}^{2}(\theta) \frac{P_{\omega}^{2}}{A}\left[\frac{\sin \left(\frac{\Delta k L}{2}\right)}{\frac{\Delta k L}{2}}\right]^{2} T_{\omega}^{2}(\theta) T_{2 \omega}(\theta)
$$


where $\theta$ is the external angle of incidence, and $L$ becomes the angle-dependent nonlinear pathlength in the sample (i.e. it is the thickness of the nonlinear region divided by $\cos \theta_{2 \omega}$ ). Equation (2.34) assumes that the material has low refractive index $\left(n_{\omega}, n_{2 \omega} \leq 2\right)$, and thus the effect of multiple reflections is neglected [21].

By appropriately choosing the orientation of the crystal principal axes, the rotation axis, and the fundamental polarization, $\chi_{\text {eff }}$ assumes a form that allows a single element of the $\chi_{i l}$ tensor to be determined. In order to illustrate how the appropriate choices of these parameters are done, consider the case of poled fused silica. Recall that the nonlinear susceptibility tensor for poled fused silica was given in equation (2.22) as:

$$
\left[\begin{array}{cccccc}
0 & 0 & 0 & 0 & \chi_{31} & 0 \\
0 & 0 & 0 & \chi_{31} & 0 & 0 \\
\chi_{31} & \chi_{31} & \chi_{33} & 0 & 0 & 0
\end{array}\right]
$$

and thus the nonlinear polarization components leading to SHG, as obtained from equation (2.19) are:

$$
\left[\begin{array}{c}
P_{x}^{2 \omega} \\
P_{y}^{2 \omega} \\
P_{z}^{2 \omega}
\end{array}\right]=\frac{\epsilon_{0}}{2}\left[\begin{array}{c}
2 \chi_{31} E_{x}(\omega) E_{z}(\omega) \\
2 \chi_{31} E_{y}(\omega) E_{z}(\omega) \\
\chi_{31} E_{x}(\omega)^{2}+\chi_{31} E_{y}(\omega)^{2}+\chi_{33} E_{z}(\omega)^{2}
\end{array}\right]
$$

Figure 2.5 shows the lab reference frame $\left(x^{\prime}-y^{\prime}-z^{\prime}\right)$ and the sample reference frame $(x-y-z)$. Let the $z^{\prime}$-axis be in the direction of light propagation, and the sample is rotated about its $y$-axis. When the sample is positioned at an angle $\vartheta$ relative to the lab frame, the relationship between the two reference frames is given by:

$$
\left[\begin{array}{l}
x^{\prime} \\
y^{\prime} \\
z^{\prime}
\end{array}\right]=\left[\begin{array}{ccc}
\cos \vartheta & 0 & \sin \vartheta \\
0 & 1 & 0 \\
-\sin \vartheta & 0 & \cos \vartheta
\end{array}\right]\left[\begin{array}{l}
x \\
y \\
z
\end{array}\right]
$$

where the matrix above is the $3 \mathrm{D}$ rotation matrix for rotating about the $y$-axis in a clockwise direction if looking in the $+y$ direction. From this equation, the following 


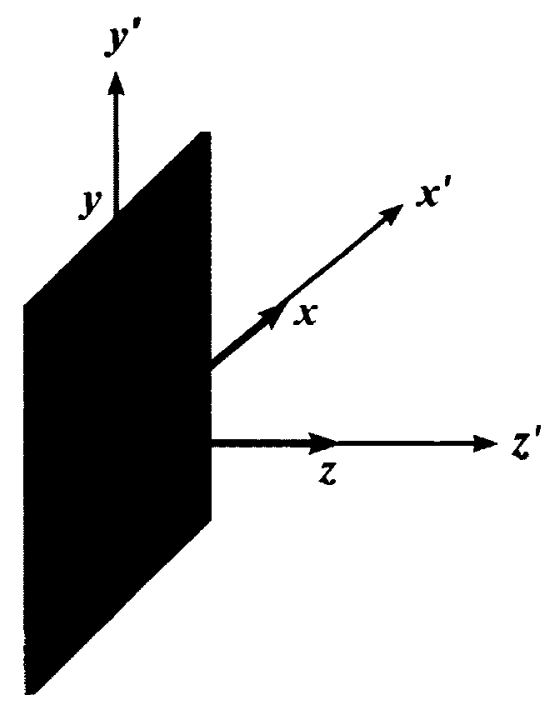

(a)

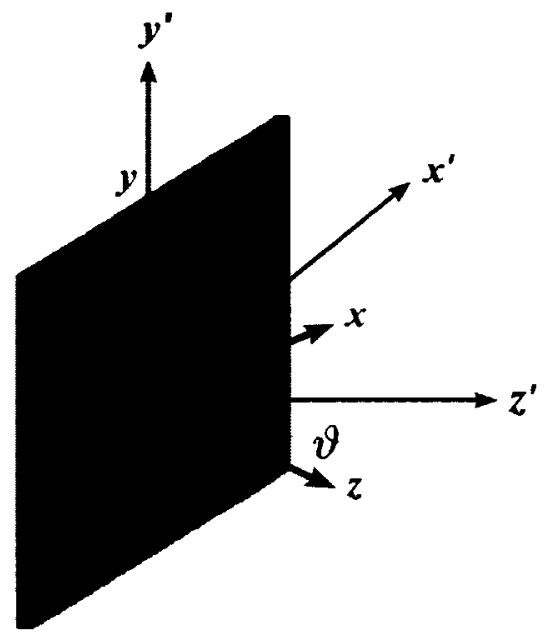

(b)

Figure 2.5: Lab reference frame $x^{\prime}-y^{\prime}-z^{\prime}$ and sample reference frame $x-y-z$. (a) Sample normal to incident beam, (b) Sample rotated at angle $\theta$ relative to incident beam.

can be obtained:

$$
\begin{array}{ll}
x^{\prime}=x \cos \vartheta+z \sin \vartheta & x=x^{\prime} \cos \vartheta-z^{\prime} \sin \vartheta \\
y^{\prime}=y & y=y^{\prime} \\
z^{\prime}=-x \sin \vartheta+z \cos \vartheta & z=x^{\prime} \sin \vartheta+z^{\prime} \cos \vartheta
\end{array}
$$

where $\vartheta$ refers to the interior propagation angle relative to the surface normal (i.e. $\left.\theta_{2 \omega} \approx \theta_{\omega}\right)$.

We now distinguish between two cases corresponding to two polarizations of the fundamental beam:

\section{Fundamental beam is $s$-polarized}

In this case, electric field of the input beam is perpendicular to the incidence plane, i.e. it is along $y^{\prime}$. Thus $E_{x}^{\prime}=0, E_{y^{\prime}}=E(\omega)$ and $E_{z^{\prime}}=0$. Using the rotation equations (2.38), this corresponds to $E_{x}=0, E_{y}=E_{y^{\prime}}$, and $E_{z}=0$, 
and therefore from equation (2.36):

$$
\left[\begin{array}{c}
P_{x}^{2 \omega} \\
P_{y}^{2 \omega} \\
P_{z}^{2 \omega}
\end{array}\right]=\frac{\epsilon_{0}}{2}\left[\begin{array}{c}
0 \\
0 \\
\chi_{31} E_{y^{\prime}}^{2}
\end{array}\right]
$$

$P_{z}$ is in the plane of incidence, therefore the SH signal is strictly $p$-polarized. If the SH signal is detected in the $x^{\prime}$-polarization, the resultant $P_{x^{\prime}}^{2 \omega}$, as obtained from equations (2.38), is:

$$
\begin{aligned}
P_{x^{\prime}}^{2 \omega} & =P_{x}^{2 \omega} \cos \vartheta+P_{z}^{2 \omega} \sin \vartheta \\
& =\frac{\epsilon_{0}}{2} \underbrace{\left[\chi_{31} \sin \vartheta\right]}_{\chi_{\epsilon \in f, s}} E^{2}(\omega)
\end{aligned}
$$

where $\chi_{e f f, s}$ is the effective nonlinear coefficient for a $s$-polarized fundamental beam, and it includes contributions from $\chi_{31}$ alone. Therefore, by using $s$ polarized fundamental beam, and detecting the $p$-polarized portion of the $\mathrm{SH}$ signal, the $\chi_{31}$ nonlinear coefficient of poled silica can be determined.

\section{Fundamental beam is $p$-polarized}

In this case, electric field of the input beam is in the incidence plane, i.e. it is along $x^{\prime}$. Thus $E_{x}^{\prime}=E(\omega)$ and $E_{y^{\prime}}=E_{z^{\prime}}=0$. Using the rotation equations (2.38), this corresponds to $E_{x}=E_{x^{\prime}} \cos \vartheta, E_{y}=0$, and $E_{z}=E_{x^{\prime}} \sin \vartheta$, and therefore:

$$
\left[\begin{array}{l}
P_{x}^{2 \omega} \\
P_{y}^{2 \omega} \\
P_{z}^{2 \omega}
\end{array}\right]=\frac{\epsilon_{0}}{2}\left[\begin{array}{c}
2 \chi_{31} E_{x^{\prime}}^{2} \sin \vartheta \cos \vartheta \\
0 \\
\chi_{31} E_{x^{\prime}}^{2} \cos ^{2} \vartheta+\chi_{33} E_{x^{\prime}}^{2} \sin ^{2} \vartheta
\end{array}\right]
$$

Both $P_{x}$ and $P_{z}$ are in the plane of incidence, therefore the SH signal is strictly $p$-polarized. If the SH signal is detected in the $x^{\prime}$-polarization, we need to obtain $P_{x^{\prime}}^{2 \omega}$ using equations 2.38 , resulting in:

$$
\begin{aligned}
P_{x^{\prime}}^{2 \omega} & =P_{x}^{2 \omega} \cos \vartheta+P_{z}^{2 \omega} \sin \vartheta \\
& =\frac{\epsilon_{0}}{2} \underbrace{\left[3 \chi_{31} \sin \vartheta \cos ^{2} \vartheta+\chi_{33} \sin ^{3} \vartheta\right]}_{\chi_{\text {t:fip }}} E^{2}(\omega)
\end{aligned}
$$


where $\chi_{e f f, p}$ is the effective nonlinear coefficient for a $p$-polarized fundamental beam, which includes contributions from $\chi_{31}$ and $\chi_{33}$. Therefore, once $\chi_{31}$ is determined from $s$-polarized fundamental beam measurements, the value of $\chi_{33}$ of poled silica can be extracted using $p$-polarized fundamental beam. Note that in both cases the SH output is $p$-polarized.

Following a similar procedure, the effective nonlinear coefficients of other crystalline materials can be derived. Table 2.2.3 summarizes the results for the various materials that will be encountered in this project.

Because the optical nonlinear coefficients are dispersive, one cannot directly compare the quantitative value of a $\chi_{i l}$ coefficient if the characterization measurements were done at different wavelengths. A way to perform wavelength scaling of the nonlinear coefficients is to use the Miller wavelength scaling rule [41]. Miller made an empirical observation that the quantity $\Delta_{i j k}$, defined by:

$$
\Delta_{i j k}=\frac{d_{i j k}\left(-\omega_{1}-\omega_{2} ; \omega_{1}, \omega_{2}\right)}{\chi_{i i}\left(\omega_{1}+\omega_{2}\right) \chi_{j j}\left(\omega_{1}\right) \chi_{k k}\left(\omega_{2}\right)}
$$

has little dispersion, varying from a constant value by only a factor $\sim 2$ for all noncentrosymmetric crystals: $d_{i j k}$ is the second-order nonlinear coefficient, and the $\chi$ 's are linear susceptibility tensor elements $\chi_{i i}(\omega)=n_{i}^{2}-1$, where $n_{i}$ is the refractive index for light of frequency $\omega$ polarized along the $i$ axis. This observation is supported by calculations of the nonlinear response of a classical anharmonic oscillator [42], which assumes that the system has a single resonant frequency. This was shown to be not a very good approximation in actual materials [37], however, a weaker form of Miller's rule, stating that a constant $\Delta_{i j k}$ is associated with each nonlinear coefficient of a particular crystal, is often used to extrapolate the $d_{i l}$ coefficients from measured to other wavelengths. As an illustration, Figure 2.6 shows the dispersion of the $d_{31}=\frac{1}{2} \chi_{31}^{(2)}$ coefficient of lithium niobate, calculated using the weaker form of Miller's rule as described above, along with a few experimentally measured values reported in [37] and [39]. The values predicted by Miller's scaling rule do not always agree well with experimental values, nonetheless it is often used in practice to get an estimate of the dispersion of the nonlinear optical coefficient. 


\begin{tabular}{|c|c|c|}
\hline \multirow[t]{3}{*}{$\begin{array}{l}\text { POLED } \\
\text { SILICA }\end{array}$} & $\begin{array}{l}\text { rotation } \\
\text { matrix: }\end{array}$ & {$\left[\begin{array}{ccc}\cos \vartheta & 0 & \sin \vartheta \\
0 & 1 & 0 \\
-\sin \vartheta & 0 & \cos \vartheta\end{array}\right]$} \\
\hline & $\begin{array}{l}s \text {-polarized } \\
\text { fundamental: }\end{array}$ & $\begin{array}{l}s \text {-polarized SH: } \chi_{e f f}=0 \\
p \text {-polarized SH: } \chi_{e f f}=\chi_{31} \sin \vartheta\end{array}$ \\
\hline & $\begin{array}{l}p \text {-polarized } \\
\text { fundamental: }\end{array}$ & $\begin{array}{l}s \text {-polarized SH: } \chi_{\text {eff }}=0 \\
p \text {-polarized SH: } \chi_{\text {eff }}=3 \chi_{31} \sin \vartheta \cos ^{2} \vartheta+\chi_{33} \sin ^{3} \vartheta\end{array}$ \\
\hline \multirow[t]{4}{*}{$\begin{array}{l}x \text {-CUT } \\
\text { QUARTZ }\end{array}$} & $\begin{array}{l}\text { rotation } \\
\text { matrix: }\end{array}$ & {$\left[\begin{array}{ccc}\cos \left(90^{\circ}+\vartheta\right) & 0 & \sin \left(90^{\circ}+\vartheta\right) \\
0 & 1 & 0 \\
-\sin \left(90^{\circ}+\vartheta\right) & 0 & \cos \left(90^{\circ}+\vartheta\right)\end{array}\right]$} \\
\hline & $\begin{array}{l}s \text {-polarized } \\
\text { fundamental: }\end{array}$ & $\begin{array}{l}s \text {-polarized SH: } \chi_{e f f}=0 \\
p \text {-polarized SH: } \chi_{e f f}=\chi_{11} \sin \vartheta\end{array}$ \\
\hline & $\begin{array}{l}p \text {-polarized } \\
\text { fundamental: }\end{array}$ & $\begin{array}{l}s \text {-polarized SH: } \chi_{\text {eff }}=2 \chi_{14} \sin \vartheta \cos \vartheta \\
p \text {-polarized SH: } \chi_{\text {eff }}=\chi_{11} \sin ^{3} \vartheta\end{array}$ \\
\hline & $\begin{array}{l}\chi_{i l} \text { values: } \\
\left(\lambda_{2 \omega}=400 \mathrm{~nm}\right)\end{array}$ & $\begin{array}{l}\chi_{11}=0.63 \mathrm{pm} / \mathrm{V} \text { (scaled from } \lambda_{2 \omega}=532 \mathrm{~nm} \text { in [37]) } \\
\chi_{14}=0.02 \mathrm{pm} / \mathrm{V} \text { (scaled from } \lambda_{2 \omega}=532 \mathrm{~nm} \text { in [22]) }\end{array}$ \\
\hline \multirow{4}{*}{$\begin{array}{l}z \text {-CUT } \\
\text { LITHIUM } \\
\text { NIOBATE }\end{array}$} & $\begin{array}{l}\text { rotation } \\
\text { matrix: }\end{array}$ & {$\left[\begin{array}{ccc}\cos \vartheta & 0 & \sin \vartheta \\
0 & 1 & 0 \\
-\sin \vartheta & 0 & \cos \vartheta\end{array}\right]$} \\
\hline & $\begin{array}{l}s \text {-polarized } \\
\text { fundamental: }\end{array}$ & $\begin{array}{l}s \text {-polarized SH: } \chi_{\text {eff }}=\chi_{22} \\
p \text {-polarized SH: } \chi_{e f f}=\chi_{31} \sin \vartheta\end{array}$ \\
\hline & $\begin{array}{l}p \text {-polarized } \\
\text { fundamental: }\end{array}$ & $\begin{array}{l}s \text {-polarized SH: } \chi_{e f f}=\chi_{22} \cos ^{2} \vartheta \\
p \text {-polarized SH: } \chi_{e f f}=3 \chi_{31} \sin \vartheta \cos ^{2} \vartheta+\chi_{33} \sin ^{3} \vartheta\end{array}$ \\
\hline & $\begin{array}{l}\chi_{i l} \text { values: } \\
\left(\lambda_{2 \omega}=400 \mathrm{~nm}\right)\end{array}$ & $\begin{array}{l}\left.\chi_{22}=6.05 \mathrm{pm} / \mathrm{V} \text { (scaled from } \lambda_{2 \omega}=532 \mathrm{~nm} \text { in }[38]\right) \\
\left.\chi_{31}=10.78 \mathrm{pm} / \mathrm{V} \text { (scaled from } \lambda_{2 \omega}=532 \mathrm{~nm} \text { in }[39]\right) \\
\chi_{33}=80.6 \mathrm{pm} / \mathrm{V} \text { (scaled from } \lambda_{2 \omega}=532 \mathrm{~nm} \text { in }[40] \text { ) }\end{array}$ \\
\hline
\end{tabular}

Table 2.2: Summary of the effective second-order nonlinear coefficients for poled silica, crystalline quartz and lithium niobate. 


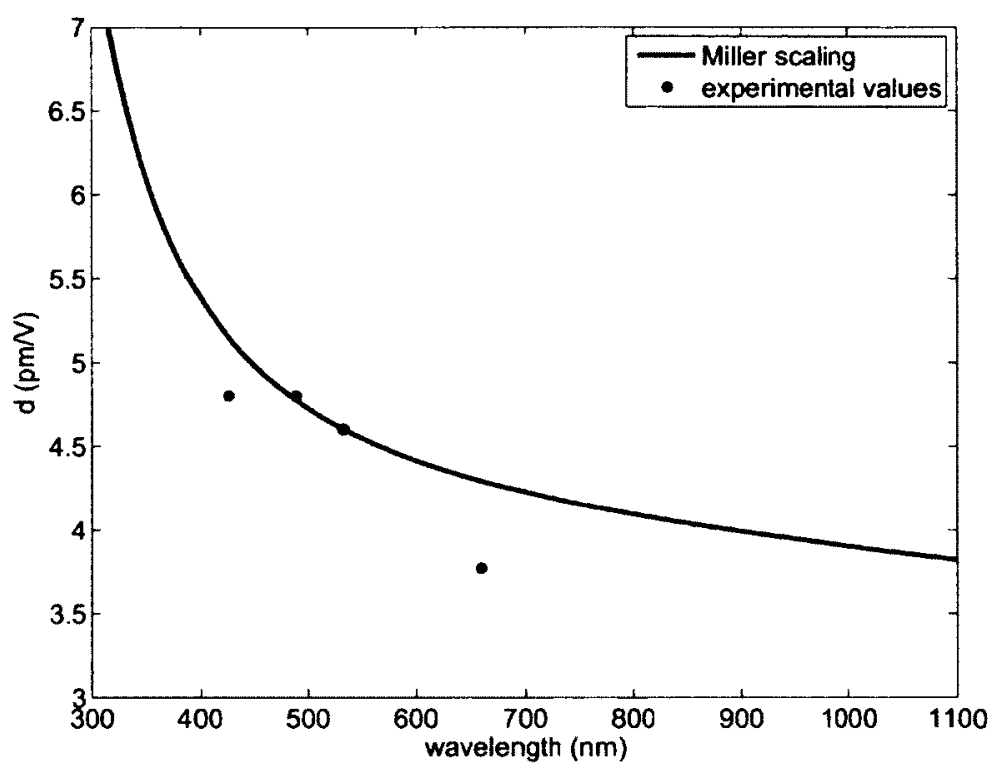

Figure 2.6: Approximated dispersion of the $d_{31}$ nonlinear coefficient of lithium niobate as calculated using Miller wavelength scaling rule. Scaling is normalized to $d_{31}$ measured at $\lambda_{2 \omega}=532 \mathrm{~nm}$, and the experimental values are taken from [37] and [39].

Because the experimental work in this project is done at the fundamental wavelength of $800 \mathrm{~nm}$ (due to the availability of the laser source), all the known reference values of the $\chi_{i l}$ coefficients in Table 2.2.3 were scaled from their measurement wavelength to $\lambda_{2 \omega}=400 \mathrm{~nm}$ using Miller wavelength scaling rule.

Now that the form of the effective nonlinear coefficient $\chi_{\text {eff }}$ in equation (2.12) is known, we can graph the expected results when the second harmonic is measured in a Maker fringe setup. Figure 2.7 shows typical results for slabs of $x$-cut quartz and $z$ cut lithium niobate. In both cases, because the nonlinear region is much greater than the coherence length $L_{c}$, rotation of the nonlinear sample reveals multiple modulation fringes that correspond to increases in path length equal to twice the coherence length. In the mathematical model used to obtain the plots in Figure 2.7 it was assumed that the samples were $0.5 \mathrm{~mm}$ thick, and the wavelength of the fundamental beam was $800 \mathrm{~nm}$, at which the coherence lengths for quartz and lithium niobate are about $10 \mu \mathrm{m}$ and $1 \mu \mathrm{m}$, respectively (calculated using equation (2.16) based on the Sellmeier equations for the two crystals). 


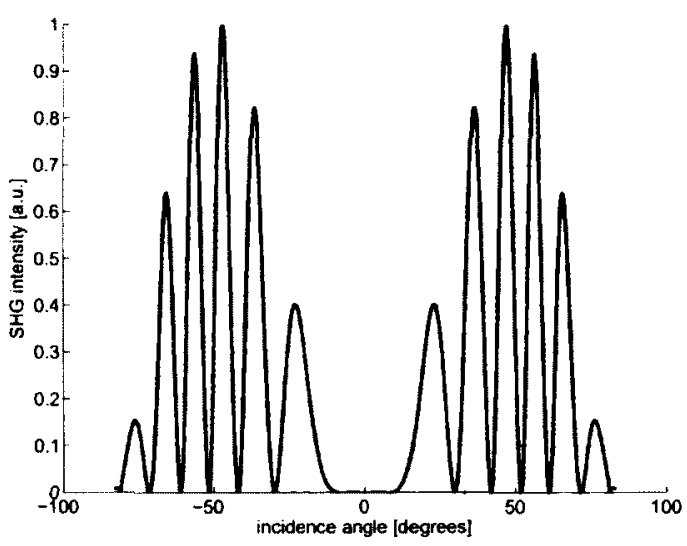

(a)

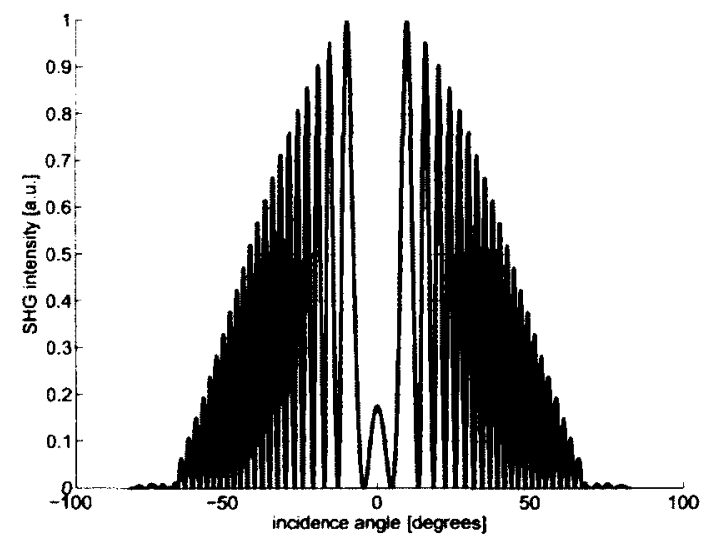

(b)

Figure 2.7: Calculated Maker fringe patterns for second harmonic generated in thick, uniformly nonlinear crystals: (a) $x$-cut quartz ( $s$-polarized fundamental, $p$-polarized $\mathrm{SH}$ ), (b) $z$-cut lithium niobate ( $s$-polarized fundamental, $s$-polarized $\mathrm{SH}$ ).

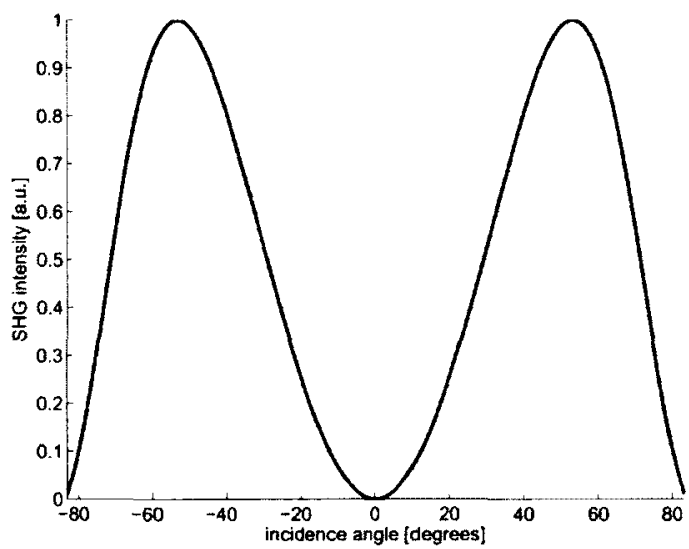

(a)

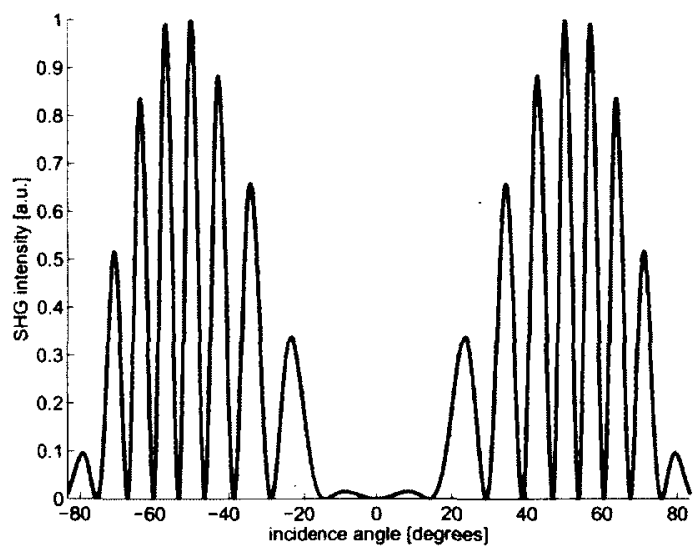

(b)

Figure 2.8: Calculated Maker fringe pattern for second harmonic generated in poled silica with nonlinear regions of different thicknesses: (a) a thin nonlinear region, (b) two thin nonlinear regions on opposite sides of the sample (the nonlinearity in both regions is assumed to be uniform and equal, and the regions separated by $0.5 \mathrm{~mm}$ ). In both cases $s$-polarized fundamental is assumed, and the $p$-polarized component of the $\mathrm{SH}$ is detected.

As will be discussed in later chapters, in the case of poled silica, the nonlinearity is induced only in certain area(s) and not throughout the entire sample. This distribution of the nonlinearity highly depends on the materials used and the experimental 
poling setup, and thus the Maker fringe results also vary. When the nonlinearity extends only on one side of the sample from $z=0$ to $z=z_{0}$ and is uniform, the expression for the SH power is similar to equation (2.34) but with $L=z_{0}$. If $z_{0}$ is smaller than the coherence length $L_{c}$, the Maker fringe measurement waveform will not have any modulation fringes, as shown in Figure 2.8(a), and only two lobes appear on either side of the normal. The sample thickness was again assumed to be $0.5 \mathrm{~mm}$ and fundamental wavelength of $800 \mathrm{~nm}$, at which the coherence length of fused silica is $11.9 \mu \mathrm{m}$ (as calculated using equation 2.16). If the nonlinearity is not uniform, the integration in equation (2.8) must take this into account.

In some poling experiments two nonlinear regions are induced near the two surfaces of the glass sample. In this case, modulation fringes will be observed because of the interference between the two nonlinear regions. Deep fringes indicate that the magnitude of the two nonlinearities is comparable, while shallow fringes mean that most of the nonlinearity is in one region. A typical Maker fringe measurement result for the case of two nonlinear regions on opposite sides of the sample is shown in Figure 2.8(b).

By comparing the second harmonic Maker fringe measurements from a sample of interest and a known reference, the value of the induced nonlinearity in the sample can be calculated. For example, by observing Table 2.2.3, it can seen that all three materials generate $p$-polarized SH for $s$-polarized fundamental beam, and all the effective nonlinear coefficients depend on a single $\chi^{(2)}$ coefficient. Therefore, by setting the fundamental beam and the SH detection to the mentioned polarizations, the Maker fringe measurement of poled silica can be compared to that of quartz or lithium niobate, and the value of the induced $\chi_{31}$ can be calculated.

Using equation (2.34), the ratio of the $\mathrm{SH}$ powers generated in a sample of interest and in a known reference is:

$$
\frac{P_{2 \omega}^{s a m}}{P_{2 \omega}^{\text {ref }}}=\left(\frac{\chi_{\text {eff }, \text { sam }}}{\chi_{\text {eff }, \text { ref }}}\right)^{2} \frac{\left(n_{\omega}^{2} n_{2 \omega}\right)_{\text {ref }}}{\left(n_{\omega}^{2} n_{2 \omega}\right)_{s a m}}\left(\frac{\Delta k_{\text {ref }}}{\Delta k_{\text {sam }}}\right)^{2}\left(\frac{B_{\text {sam }}}{B_{\text {ref }}}\right)\left(\frac{\sin \left(\frac{\Delta k L}{2}\right)_{s a m}}{\sin \left(\frac{\Delta k L}{2}\right)_{r e f}}\right)^{2} \frac{\left(T_{\omega}^{2} T_{2 \omega}\right)_{s a m}}{\left(T_{\omega}^{2} T_{2 \omega}\right)_{r e f}}
$$

If any one of the signals is measured at the maximum of a modulation fringe (in 
which case the nonlinear interaction length is equivalent to a single coherence length), the value of the corresponding $\sin \left(\frac{\Delta k L}{2}\right)$ term in the above equation reduces to 1 . However, if the measured Maker fringe pattern has no modulation fringes, in order to calculate the induced nonlinearity in the sample of interest, the depth of the nonlinearity $(L)$ must be known. In general the sample and the reference Maker fringe measurements are not done at the same rotation angle (because we choose a maximum of a modulation fringe in each case), therefore the variation in the beam spot size at different angles is accounted for by the $B_{\text {sam }} / B_{\text {ref }}$ term.

\subsection{Electro-Optic Modulation}

The presence of a second-order nonlinearity allows not only its characterization by second harmonic generation, but also permits electro-optic modulation of light. The linear electro-optic (Pockels) effect described in this section refers to a linear change in the refractive index of a nonlinear material in the presence of an electric field. Because a high intensity optical field is not required to observe this effect, studies of the electro-optic effect preceded the laser (and thus the development of the field of nonlinear optics in general), and thus it is typically quantified by the electro-optic tensor $r$ rather than the nonlinear susceptibility tensor $\chi^{(2)}$.

The optical properties of an anisotropic medium can be characterized by a useful mathematical construct called the index ellipsoid. When the axes of the ellipsoid correspond to the principal axes of the anisotropic medium, it takes the form:

$$
\frac{x^{2}}{n_{x}^{2}}+\frac{y^{2}}{n_{y}^{2}}+\frac{z^{2}}{n_{z}^{2}}=1
$$

where the directions $x, y$ and $z$ are the principal axes, and $n_{x}, n_{y}$ and $n_{z}$ are the principal refractive indices. The linear electro-optic coefficient $r_{i j k}$ has been traditionally defined as [24]:

$$
\Delta\left(\frac{1}{n^{2}}\right)_{i j} \equiv r_{i j k} E_{k}
$$

where $E_{k}$ is the $k$-component of the electric field, $k=x, y, z$, and summation over repeated indices is assumed. Using the formalism described in section 2.1 for the fre- 
quency domain second-order nonlinear polarization, it can be shown that the relationship between the electro-optic coefficient and the second-order nonlinear susceptibility is $[21]$ :

$$
r_{i j k}=\frac{2 \chi_{i j k}^{(2)}(-\omega ; \omega, 0)}{n_{i}^{2} n_{j}^{2}}
$$

where $n_{i}$ and $n_{j}$ are the principal refractive indices. Although this expression points to the common underlying origin of the linear electro-optic effect and the second-order nonlinearity of the medium, it is often not consistent with experimentally observed values of $\chi_{i j k}$ and $r_{i j k}$. This is because the expression assumes that the medium is nondispersive - an assumption that is clearly not satisfied when one of the components is at an optical frequency $\omega$ and the other is a steady field with zero frequency.

Analogously to the susceptibility tensor case described in section 2.2 .2 , the notation can be simplified by reducing the $r_{i j k}$ tensor to a $6 \times 3 r_{l k}$ matrix using the following new indices:

$\begin{array}{ccccccc}i j: & 11 & 22 & 33 & 23,32 & 31,13 & 12,21 \\ l: & 1 & 2 & 3 & 4 & 5 & 6\end{array}$

such that equation (2.46) becomes:

$$
\left[\begin{array}{l}
\Delta\left(\frac{1}{n^{2}}\right)_{1} \\
\Delta\left(\frac{1}{n^{2}}\right)_{2} \\
\Delta\left(\frac{1}{n^{2}}\right)_{3} \\
\Delta\left(\frac{1}{n^{2}}\right)_{4} \\
\Delta\left(\frac{1}{n^{2}}\right)_{5} \\
\Delta\left(\frac{1}{n^{2}}\right)_{6}
\end{array}\right]=\left[\begin{array}{lll}
r_{11} & r_{12} & r_{13} \\
r_{21} & r_{22} & r_{23} \\
r_{31} & r_{32} & r_{33} \\
r_{41} & r_{42} & r_{43} \\
r_{51} & r_{52} & r_{53} \\
r_{61} & r_{62} & r_{63}
\end{array}\right]\left[\begin{array}{c}
E_{x} \\
E_{y} \\
E_{z}
\end{array}\right]
$$

The change in the refractive indices of the normal modes of propagation due to an applied electric field described by equation (2.48) can exist only in materials that do not possess inversion symmetry. Following a similar argument to the one presented for the susceptibility coefficient $\chi^{(2)}$ in section 2.1 , the requirement for the invariance of the physical properties under an inversion symmetry transformation for centrosymmetric materials can only be satisfied if the electro-optic coefficient is zero. 
While the principal-axes index ellipsoid is given in equation (2.45), the equation of the index ellipsoid in the presence of an external electric field takes the general ellipsoid form:

$$
\begin{aligned}
{\left[\frac{1}{n_{x}^{2}}+\Delta\left(\frac{1}{n^{2}}\right)_{1}\right]_{x^{2}} } & +\left[\frac{1}{n_{y}^{2}}+\Delta\left(\frac{1}{n^{2}}\right)_{2}\right] y^{2}+\left[\frac{1}{n_{z}^{2}}+\Delta\left(\frac{1}{n^{2}}\right)_{3}\right]^{2} \\
& +2 y z \Delta\left(\frac{1}{n^{2}}\right)_{4}+2 z x \Delta\left(\frac{1}{n^{2}}\right)_{5}+2 x y \Delta\left(\frac{1}{n^{2}}\right)_{6}=1
\end{aligned}
$$

When combined with equation (2.48), this can be rewritten as:

$$
\begin{aligned}
{\left[\frac{1}{n_{x}^{2}}+r_{1 k} E_{k}\right] x^{2} } & +\left[\frac{1}{n_{y}^{2}}+r_{2 k} E_{k}\right] y^{2}+\left[\frac{1}{n_{z}^{2}}+r_{3 k} E_{k}\right] z^{2} \\
& +2 y z r_{4 k} E_{k}+2 z x r_{5 k} E_{k}+2 x y r_{6 k} E_{k}=1
\end{aligned}
$$

when again summation over repeated indices is assumed. As expected, when the electric field vanishes, the ellipsoid described above reduces to the unperturbed ellipsoid given by equation (2.45). When one or more of the "mixed" terms in equation (2.50) are present, the major axes of the ellipsoid are no longer parallel to the principal axes $x, y$ and $z$. The problem then is to find a new coordinate system, in which the equation of the ellipsoid contains no mixed terms, i.e. a new coordinate system that defines the directions of the major axes of the ellipsoid in the presence of an external field.

Similarly to the case of the $\chi^{(2)}$ susceptibility tensor, the symmetry of the reduced electro-optic tensor for poled silica matches that of the $\infty \mathrm{mm}$ (as well as $6,6 \mathrm{~mm}, 4$ and $4 \mathrm{~mm}$ ) group, i.e.:

$$
\left[\begin{array}{ccc}
0 & 0 & r_{13} \\
0 & 0 & r_{13} \\
0 & 0 & r_{33} \\
0 & r_{13} & 0 \\
r_{13} & 0 & 0 \\
0 & 0 & 0
\end{array}\right]
$$

and the corresponding reduced electro-optic tensors for crystalline quartz and lithium 
niobate are given by:

$$
\left[\begin{array}{ccc}
r_{11} & 0 & 0 \\
-r_{11} & 0 & 0 \\
0 & 0 & 0 \\
r_{41} & 0 & 0 \\
0 & -r_{41} & 0 \\
0 & -r_{11} & 0
\end{array}\right]
$$

and

$$
\left[\begin{array}{ccc}
0 & -r_{22} & r_{13} \\
0 & r_{22} & r_{13} \\
0 & 0 & r_{33} \\
0 & r_{51} & 0 \\
r_{51} & 0 & 0 \\
-r_{22} & 0 & 0
\end{array}\right]
$$

respectively.

For poled glass, equation (2.48) takes the form:

$$
\left[\begin{array}{c}
\Delta\left(\frac{1}{n^{2}}\right)_{1} \\
\Delta\left(\frac{1}{n^{2}}\right)_{2} \\
\Delta\left(\frac{1}{n^{2}}\right)_{3} \\
\Delta\left(\frac{1}{n^{2}}\right)_{4} \\
\Delta\left(\frac{1}{n^{2}}\right)_{5} \\
\Delta\left(\frac{1}{n^{2}}\right)_{6}
\end{array}\right]=\left[\begin{array}{ccc}
0 & 0 & r_{13} \\
0 & 0 & r_{13} \\
0 & 0 & r_{33} \\
0 & r_{13} & 0 \\
r_{13} & 0 & 0 \\
0 & 0 & 0
\end{array}\right]\left[\begin{array}{c}
E_{x} \\
E_{y} \\
E_{z}
\end{array}\right]=\left[\begin{array}{c}
r_{13} E_{z} \\
r_{13} E_{z} \\
r_{33} E_{z} \\
r_{13} E_{y} \\
r_{13} E_{x} \\
0
\end{array}\right]
$$

therefore the corresponding index ellipsoid in the presence of an external electric field is:

$$
\begin{aligned}
{\left[\frac{1}{n^{2}}+r_{13} E_{z}\right] x^{2} } & +\left[\frac{1}{n^{2}}+r_{13} E_{z}\right] y^{2}+\left[\frac{1}{n^{2}}+r_{33} E_{z}\right] z^{2} \\
& +2 y z r_{13} E_{y}+2 z x r_{13} E_{x}=1
\end{aligned}
$$

where $n_{x} \approx n_{y} \approx n_{z} \equiv n$ is assumed since the induced birefringence in poled glasses is usually very low. If the applied electric field $E$ is parallel to the $z$-axis, equation (2.55) 
becomes:

$$
\left[\frac{1}{n^{2}}+r_{13} E\right] x^{2}+\left[\frac{1}{n^{2}}+r_{13} E\right] y^{2}+\left[\frac{1}{n^{2}}+r_{33} E\right] z^{2}=1
$$

Since there are no mixed terms, the principal axes of the new index ellipsoid remain the same, but their lengths become:

$$
\begin{aligned}
& n_{x^{\prime}}=n_{y^{\prime}} \approx n-\frac{n^{3}}{2} r_{13} E \\
& n_{z^{\prime}} \approx n-\frac{n^{3}}{2} r_{33} E
\end{aligned}
$$

where it was assumed that $r_{13} E$ and $r_{33} E$ are much smaller than $1 / n^{2}$, and thus the approximation $1 / \sqrt{1+x} \approx 1-x / 2$ for small $x$ was used [17].

When a modulator is constructed on the basis of the electro-optic effect, the electric field $E$ may be applied in a direction parallel to the direction of light propagation (longitudinal modulator) or perpendicular to it (transverse modulator). For the longitudinal phase modulator, illustrated in Figure 2.9(a), $E=V / L$, and when the light propagates over a distance $L$, the acquired phase $\phi=k L=2 \pi n(E) L / \lambda$ is equal to:

$$
\begin{aligned}
\phi & =\frac{2 \pi L}{\lambda}\left(n-\frac{n^{3}}{2} r_{13} E\right) \\
& =\underbrace{\frac{2 \pi L}{\lambda} n}_{\phi_{0}}-\underbrace{\frac{\pi n^{3} r_{13}}{\lambda} V}_{\Delta \phi}
\end{aligned}
$$

Therefore, the half-wave voltage $V_{\pi}$, defined as the applied voltage at which the phase changes by $\pi$, is given by:

$$
V_{\pi}=\frac{\lambda}{n^{3} r_{13}}
$$

For the case of a transverse phase modulator, shown in Figure 2.9(b), the voltage is applied perpendicular to the direction of light propagation. This is a more common mode of operation because the electrodes do not interfere with the optical beam, and the induced phase shift can be increased by using a longer $L$ without affecting the 
applied field, which remains $E=V / d$. In this case, the acquired phase is:

$$
\begin{aligned}
\phi & =\frac{2 \pi L}{\lambda}\left(n-\frac{n^{3}}{2} r_{13} E\right) \\
& =\underbrace{\frac{2 \pi L}{\lambda} n}_{\phi_{0}}-\underbrace{\frac{\pi L n^{3} r_{13}}{\lambda d} V}_{\Delta \phi}
\end{aligned}
$$

and the half-wave voltage is:

$$
V_{\pi}=\frac{d}{L} \frac{\lambda}{n^{3} r_{13}}
$$

A transverse modulator can also be constructed as an integrated optical device where coplanar electrodes are used, as shown in Figure 2.9(c). The electric field is applied across a waveguide for which $d \ll L$ and thus low half-wave voltages can be achieved.

When the transit time of the light propagating in the nonlinear material is small compared to the modulation period, a simple lumped electrode configuration, as was shown in Figure 2.9(c), can be used. However, this configuration has a few limiting factors that prevent it from modulating the optical signal at high frequencies. First, when the optical transit time and the period of the modulating signal become comparable, the light wave does not experience the same modulating field as it propagates through the electro-optic material, which results in a reduction in the modulation depth [43]. Consequently, a minimum required modulation depth sets a limit on the highest useful modulation frequency (typically below a few $\mathrm{GHz}$ ). The second limiting factor becomes evident when an equivalent circuit of the lumped electrode configuration is considered. The modulation frequency bandwidth, given by:

$$
\Delta f=\frac{1}{2 \pi R_{L} C}
$$

is limited by the capacitance of the formed parallel plate capacitor $(C)$ and a parallel load resistance $\left(R_{L}\right)$, which is typically inserted so that most of the modulation voltage appears across the nonlinear material and not across the internal resistance of the modulation source [43]. Decreasing the electrode length or increasing the electrode gap will decrease $C$, and hence increase $\Delta f$, but at the expense of requiring a higher drive voltage. This bandwidth-voltage tradeoff also sets a limit on the maximum usable modulation frequency. 


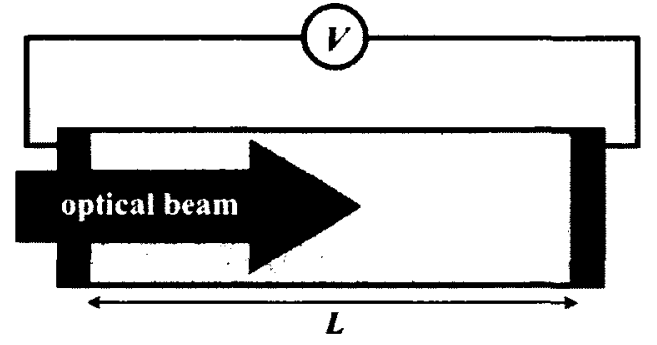

(a)

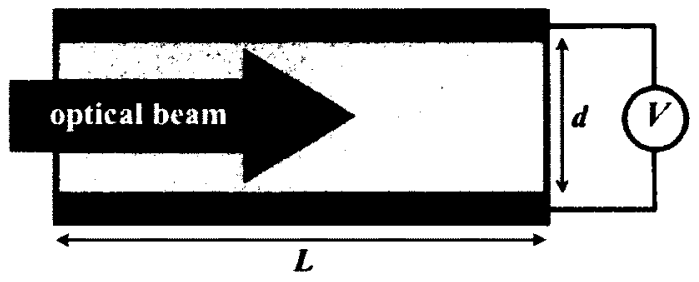

(b)

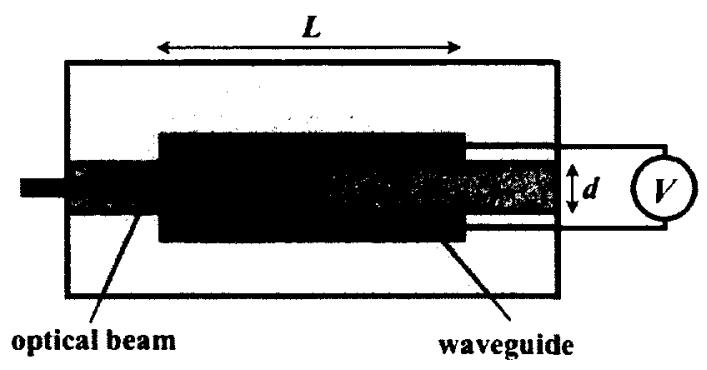

(c)
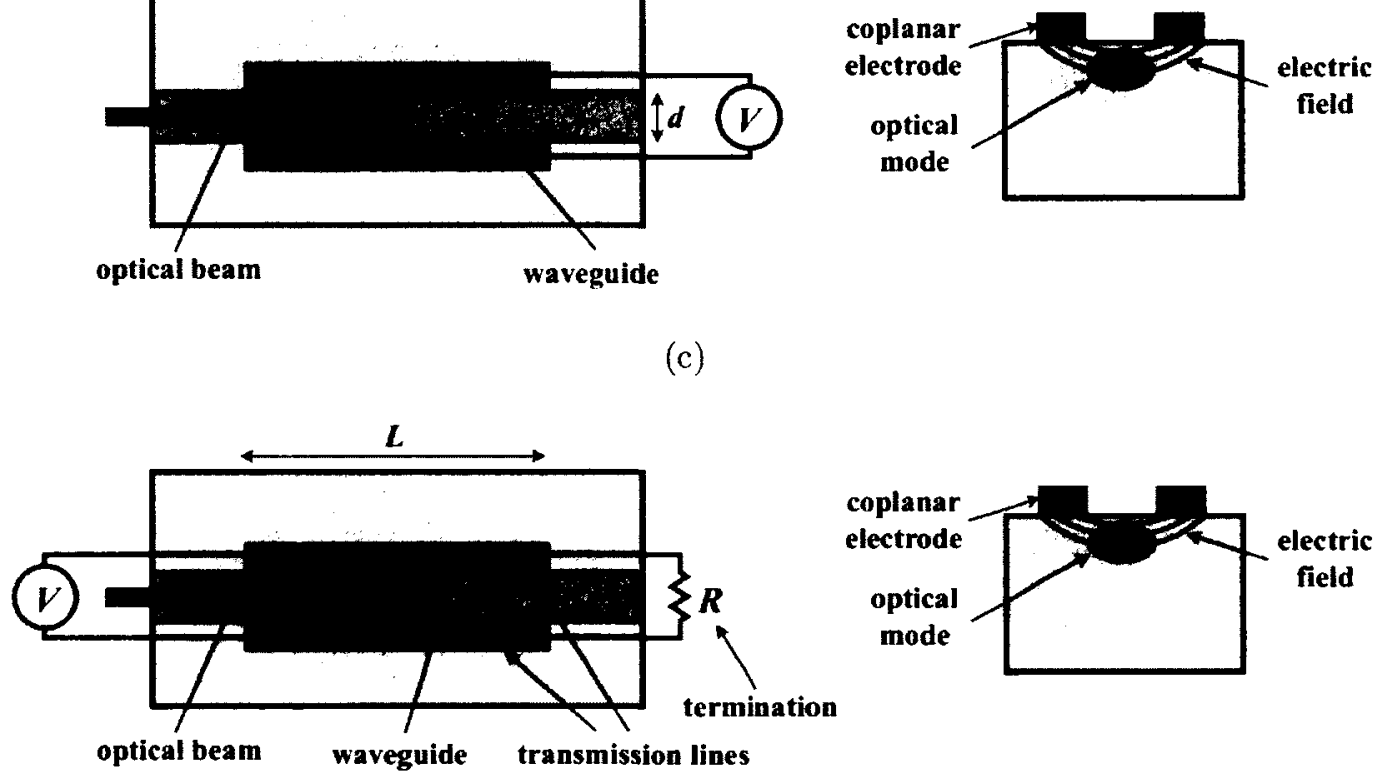

(d)

Figure 2.9: Different implementations of the electro-optic phase modulator, and the corresponding cross-sections illustrating the propagating optical mode and the electric field: (a) a longitudinal modulator. (b) a transverse modulator, (c) an integrated transverse modulator, (d) a traveling-wave transverse modulator. 
In order to overcome these two limitations and achieve modulation frequencies in the microwave or millimeter-wave ranges (10 GHz and beyond), a traveling-wave configuration can be used [44]. In this case the electrodes are made out of strip transmission lines, as illustrated in Figure 2.9(d), and the modulation voltage is applied as a traveling wave, which propagates collinearly with the optical wave. In theory, if the optical and the modulation waves have equal phase velocities, the transit time problem discussed above is eliminated and the modulation bandwidth $\Delta f$ has no limitations. In practice, phase-velocity mismatch between the optical and the modulating waves as well as the electrical attenuation of the electrodes do restrict the maximum modulation frequency. However, for a given drive voltage the travelingwave configuration can achieve a much higher modulation frequency compared to the lumped electrode configuration. Continuous work on the development of phase velocity-matching schemes and the reduction of losses of the transmission lines at high frequencies [45] [46] [47] has resulted in the high-speed electro-optical modulators that are in use today.

An important factor that affects the efficiency of the electro-optic phase modulation is the spatial overlap between the electric field that is established between the electrodes and the optical field that propagates through the modulator. An estimate of the effect of this factor is provided by the overlap integral $\Gamma$, which requires a knowledge of both the optical field and the electric field distributions [44]. The optical field profile can be computed based on theoretical calculations or using a simulation based on the geometrical and optical properties of the structure, while the calculation of the electrical field distribution can be done using numerical techniques such as conformal mapping or the finite element method. The overlap integral factor $\Gamma$ is defined as [48] [49]:

$$
\Gamma=\frac{d}{V} \frac{\iint E_{o p}^{2}(x, y) E_{e l}(x, y) d x d y}{\iint E_{o p}^{2}(x, y) d x d y}
$$

where $d$ is the inter-electrode spacing, $V$ is the applied voltage, $E_{o p}$ is the optical field, and $E_{e l}$ is the modulating electric field. In the case of longitudinal and transverse 
modulators illustrated in Figure 2.9(a) and Figure 2.9(b), the electric field distribution through the waveguiding region is uniform, with a perfect overlap between the electric field and the optical mode. Therefore $\Gamma=1$ and the induced refractive index change is given by equations (2.59)-(2.62). On the other hand, the electric field distribution obtained from coplanar electrodes in the modulator design shown in Figure 2.9(c) is not uniform over the optical field, and therefore the magnitude of the electro-optically induced phase shift, as given by equations (2.59) and (2.61), is reduced by the overlap integral factor $\Gamma[50]$. In this case the optimization of the induced index change is done through careful design of the electrode and the waveguide structures [51].

Electro-optic amplitude modulation can be realized by placing an electro-optic phase modulator in one arm of an interferometer. Because the phase shift acquired by the modulated arm can be controlled by the applied electric field, the entire device functions as an intensity modulator or a switch. The same effect can be obtained by placing an electro-optic phase modulator between crossed polarizers. The electro-optic effect is used in many other applications, such as electrically controlled directional couplers or spatial light modulators. 


\section{Chapter 3}

\section{Inducing Second-Order Nonlinearities in Glasses}

The work on inducing second-order nonlinearities (SON) in glasses began in the 1980s, following the first demonstrations of photoinduced second harmonic generation in optical fibers [52] [4]. The idea that the symmetry of the glass can be broken motivated many to explore the best methods to accomplish this and to understand the underlying physical mechanisms that are responsible for the induced nonlinear effect. This chapter gives a detailed review of the research work done in this area to date. Section 3.1 begins with the original experiments that sparked the work on poled glasses. It then gives an overview of the different poling methods that have been developed, and describes the models that are believed to be responsible for the induced nonlinearity. Section 3.2 provides a summary of the characterization methods used to obtain information about the nonlinearity in glasses, while section 3.3 describes the electro-optic modulators and switches that have been realized in poled glass-based devices. The focus of section 3.4 is on the work involving interfaces within glass structures, which play an important role during poling. Finally, in section 3.5 the idea of this research project is explained, along with its underlying hypotheses and potential applications. 


\subsection{Poling Methods}

Glass has an amorphous structure, and as a result it is a centrosymmetric material. As was shown in section 2.1, centrosymmetric materials do not possess any evenorder nonlinear properties, and thus cannot exhibit any second harmonic generation (SHG). Yet, throughout the 1980s, a number of researchers observed significant SHG coming from silica-based optical fibers when strong IR light was coupled into the fibers [52] [4]. This experimental process was later termed optical poling or photoinduced second harmonic generation. The procedure consisted of launching a high-power, pulsed, $1.06 \mu \mathrm{m}$ fundamental beam into a germanium-doped optical fiber. After about an hour, second harmonic light was detected at the output of the fiber, and its intensity grew exponentially until saturating after a few hours. After such preparation, the fiber could produce second harmonic light immediately upon re-illumination at the same fundamental wavelength. Experimental evidence suggests that the mechanism responsible for this process may be attributed to a coherent photovoltaic effect, through which a spatially modulated static electric field is established along the fiber [53] [54]. This electric field couples with the third-order nonlinearity of the glass $\chi^{(3)}$ to give an effective $\chi^{(2)}$. Essentially, this process has an inherent ability to create the conditions for quasi-phase matching, thus allowing long interaction lengths. Even though the induced SON using optical poling was quite weak $\left(\chi^{(2)} \sim 10^{-3} \mathrm{pm} / \mathrm{V}[55]\right)$, the discovery of this phenomenon motivated many to continue searching for alternative methods of inducing nonlinearity in glasses.

A significant breakthrough occurred in 1991 when a large and permanent secondorder nonlinearity was induced in a poled bulk silica sample by a temperature / static electric field poling process [5]. This process is now known as thermal poling, and it involves heating the sample to $\sim 300{ }^{\circ} \mathrm{C}$, and applying a high DC electric field (3-5 $\mathrm{kV}$ ) across the sample. This state is maintained for a certain duration (typically 5-30 minutes), at which point the heating is turned off. allowing the sample to cool with the high electric field still applied. The field is only removed when the sample reaches room temperature. The nonlinearity that was induced using the thermal 
poling method, as deduced from the second harmonic generated in the silica sample by the Maker fringe technique, was found to be a few orders of magnitude larger than in the case of optical poling [5]. The results were readily reproduced by other researchers, with a typical nonlinearity of $\chi^{(2)} \sim 1 \mathrm{pm} / \mathrm{V}$ induced in a region extending over 4-10 $\mu \mathrm{m}$ near the surface that was in contact with the anode poling electrode [5] [56] [57] [58]. Although the $1 \mathrm{pm} / \mathrm{V}$ value of the induced nonlinearity coefficient is significantly less than that of the lithium niobate crystal $\left(\chi_{33}^{(3)} \sim 80 \mathrm{pm} / \mathrm{V}\right)$, it is comparable to that of other widely-used nonlinear crystals such as quartz or KDP.

As of today, the mechanism that is responsible for the creation of the nonlinearity in thermally poled glasses is still not clear. From earlier studies of silicate glasses it is known that when a high voltage is applied to silica at elevated temperatures, impurity alkali ions (such as $\mathrm{Na}^{+}$and $\mathrm{Li}^{+}$) that are usually bonded to negatively charged non-bridging oxygen sites, drift as a result of the applied field [59]. Based on this observation, it has been suggested that, during the poling process, sodium impurity ions drift towards the cathode, and, assuming a blocking electrode condition at the anode (i.e. zero ionic conductivity), leave a negatively-charged depletion region near the anodic surface [5]. This depletion region is free of mobile charges, thus its resistivity is much higher than the rest of the glass, and as a result the applied voltage drops mainly across this region. At this stage, two mechanisms were proposed:

1. The high electric field at the glass-anode boundary region causes the formation of a positive charge layer in the depletion region. When the anode electrode allows contact of the glass with the atmosphere (i.e. it acts as non-blocking electrode), this layer consists of hydrogenated ions (e.g. $\mathrm{H}^{+}$or $\mathrm{H}_{3} \mathrm{O}^{+}$) produced by high field ionization at the glass-anode interface [60] [61] [62]. In the case when the anode prevents the contact of glass with the atmosphere (i.e. the blocking electrode condition), it forms due structural rearrangements and release of nonbridging oxygen atoms within the glass region near the anode [63]. Once the sample is cooled, the ions remain frozen in their positions. As a result, a high frozen-in electric field is established between the positive charge layer and the negatively charged depletion region (a similar field may be established near the cathode, as well, due to accumulation of the migrated impurity ions). Coupling 
between this frozen-in field and the intrinsic third-order nonlinearity of the glass results in an effective second-order nonlinearity [64].

2 . The elevated temperature increases the mobility of the non-bridging oxygen dipoles, and they are oriented in a certain direction due to the applied electric field [64] [65]. When the sample is cooled, the dipoles remain frozen in their new orientation, thus breaking the symmetry of the glass and allowing for secondorder nonlinearity.

A general expression for the effective $\chi^{(2)}$ that includes the effect of the two mechanisms is given by [65]:

$$
\chi_{e f f}^{(2)}=3 \chi^{(3)} E_{d c}+\frac{N p \beta}{5 k T} E_{d c}
$$

where the first term accounts for the interaction between $E_{d c}$ (the frozen-in electric field) and the third-order nonlinearity $\chi^{(3)}$, and the second term describes the electric field-induced orientation of the molecular second-order hyperpolarizability $\beta$. $p$ is the permanent dipole moment associated with the bond, $N$ is the concentration of orientable moieties, $k$ is Boltzmann constant, and $T$ is the temperature in Kelvins. Most of the experimental evidence seems to support the frozen-in electric field model as the mechanism responsible for the induced nonlinearity: the nonlinear region was confirmed to be depleted of alkali ions [66], a large electric field was shown to exist within the depletion region [61], a positively charged layer was detected at the anodic surface [60] [67], and the $\chi^{(2)}$ tensor symmetry was shown to be consistent with that of $\chi^{(3)}$ in silica [68]. Moreover, there is a remarkable agreement between the experimentally measured values of $\chi_{e f f}^{(2)}$ and the maximum achievable value predicted by the frozen-in electric field model - if the value of the breakdown strength of silica $\left(\sim 10^{9} \mathrm{~V} / \mathrm{m}[69]\right)$ is taken as the upper limit for $E_{d c}$ and using $\chi^{(3)}=2-3.8 \times 10^{-22}(\mathrm{~m} / \mathrm{V})^{2}$ for silica glass [70] [71], the predicted value of the second-order nonlinearity coefficient lies in the range $0.6-1.1 \mathrm{pm} / \mathrm{V}$. At the same time, the experimental results do not exclude an additional contribution of the dipole orientation mechanism [62], and in some cases directly support it [72].

In order to increase the interaction length with the induced nonlinear region, much work has been done on thermally poled silica fibers. The poling is usually 
accomplished by using specially designed D-shaped fibers or twin-hole fibers. In a Dshaped fiber, one of the poling electrodes is placed in contact with the flat fiber surface (in close proximity to the fiber core), and the other is positioned on the other side of the fiber [73] or in a hole in the fiber cladding [74]. A twin-hole fiber contains two large air holes in the cladding along the fiber, into which metal wires are inserted [75]. Time evolution of the second-order nonlinearity induced in a thermally poled Dshaped fiber was studied in [16], where it was shown that the magnitude of $\chi^{(2)}$ reached $\sim 0.64 \mathrm{pm} / \mathrm{V}$ for short poling durations, and then began to decrease for longer poling times. A twin-hole thermally poled fiber was used in [76] to realize broadly tunable second harmonic generation in a periodically poled silica fiber by utilizing mechanical compression tuning of the quasi-phase-matched periodic structure. The poled structure was achieved by uniform thermal poling, and subsequent point-bypoint UV erasure of the nonlinearity. A $32 \mathrm{~cm}$-long fiber fabricated and periodically poled using the same technique was used to achieve record-high second harmonic conversion efficiency of $15 \%$ in [77], with predicted increase to $50 \%$ for a longer device. The use of periodically poled fibers opened the door to many additional experiments to study the properties of the induced nonlinearity and to precisely control the SHG process. Very recently the measurements of the individual $\chi^{(2)}$ tensor components in a birefringent periodically poled silica fiber were used to demonstrate that the $\chi^{(2)}$ tensor symmetry is consistent with that of $\chi^{(3)}$ in silica, providing experimental evidence that the $\chi^{(2)}$ originates from a $\chi^{(3)}$ process [68]. A similar poling technique was employed in [78] to show that twisting of a birefringent periodically poled fiber can result in second harmonic phase-matching schemes that are not permitted in an untwisted fiber. On a more practical side, thermal poling and point-by-point erasure of the nonlinearity by UV irradiation were used to realize chirped-period poling, which can be used to increase the acceptance bandwidth of a quasi-phase-matched poled silica fiber [79].

Many other poling methods were explored in an effort to improve upon the thermal poling-induced nonlinearity. One method often used with polymers is corona poling, a variation of thermal poling, where the anode electrode is replaced by a tungsten needle held $\sim 1 \mathrm{~cm}$ above the sample. Corona poling of Corning 7059 glass films [6] 
and silica glass films [80] resulted in induced nonlinearity of $\chi^{(2)} \sim 0.5 \mathrm{pm} / \mathrm{V}$ and $\chi^{(2)} \sim 0.06 \mathrm{pm} / \mathrm{V}$, respectively, with the structures showing time-dependent decay of the SON coefficient [81] [82] [80]. When thermal and corona poling were compared in [83] it was concluded that a depletion region is not formed during corona poling of bulk glass, therefore the poling voltage is distributed across the entire bulk of the sample, resulting in a much weaker SON compared to thermally poled samples.

Another variation of thermal poling is $\mathrm{CO}_{2}$ laser-assisted poling, where the source of heat is provided by a $\mathrm{CO}_{2}$ laser beam [84] [85]. This method of heating is very rapid (on the order of a few seconds, compared to tens of minutes in thermal poling) and localized, thus allowing the possibility of fabricating periodically poled structures for quasi-phase matching. The induced nonlinearity using $\mathrm{CO}_{2}$ laser-assisted poling was shown to be similar to that obtained with thermal poling [7].

The method of UV poling involves irradiation by nanosecond UV laser pulses in the presence of an applied electric field [8]. In [86], an induced nonlinear coefficient of $\chi^{(2)}=6.8 \mathrm{pm} / \mathrm{V}$ was reported in UV-poled highly Ge-doped fused silica glass, a value almost an order of magnitude higher than that induced with other poling methods. The enhancement was attributed to an increase in the third-order nonlinearity of the material due to the formation of crystallites in the glass [87]. However, such high induced nonlinearities were not reproducible by other research groups, suggesting that material composition and preparation were crucial factors in this experiment. UV-fs poling is a variation on the UV poling technique, where high-intensity femtosecond UV source is used to irradiate the sample in a presence of a high electric field [9]. Two-photon absorption of femtosecond UV pulses is believed to be the mechanism responsible for the creation of a permanent depletion region within the glass, resulting in an induced second-order nonlinearity of $\sim 0.02 \mathrm{pm} / \mathrm{V}$. It is predicted that if the same experiment is performed in twin-hole fibers [88], where the maximum applied field is limited only by the dielectric strength of silica, $\chi^{(2)}$ closer to $1 \mathrm{pm} / \mathrm{V}$ could be obtained.

In electron-beam poling electrons are implanted within the glass by irradiating it with a low-energy electron beam [10]. This method has been previously used for poling polymers and for creating periodically inverted domains in lithium niobate 
crystals. Injection of electrons into dielectrics leads to the formation of an electrostatic field directed perpendicular to the surface of the sample. Similarly to the frozen-in field mechanism in thermal poling, this electrostatic field can act on the third-order susceptibility to create an effective second-order susceptibility. $\chi^{(2)} \sim 0.7 \mathrm{pm} / \mathrm{V}$ was achieved in electron-beam poled lead silicate glasses [10], a value later increased to $4 \mathrm{pm} / \mathrm{V}$ in [89], where it was shown that the induced nonlinearity increases linearly with the lead percentage in the glass. However, this method does not induce any nonlinearity in pure silica, and actually causes erasure of thermally-induced nonlinearity already present in the glass [56]. The high resolution of the electron-beam can be used to create complex periodic patterns in devices employing quasi-phase matching (either by periodically inducing localized nonlinearity, or by periodic erasure of pre-existing nonlinearity).

Unlike electron-beam poling, it was shown in [11] that proton-implantation poling can be used to induce nonlinearity on the order of $1 \mathrm{pm} / \mathrm{V}$ in pure silica glasses. Other irradiation-based poling methods that were tried with silica glasses include $\mathbf{x}$-ray poling [90] and $\gamma$-ray poling [91], both resulting in $\chi^{(2)}$ value lower than with thermal poling.

\subsection{Characterization Methods}

In order to be able to improve and engineer poled glass devices, it is essential to be able to precisely characterize the induced nonlinear effect. The ability to measure the profile, thickness, location and dynamics of the depletion region that is responsible for the induced nonlinearity is particularly important. This section provides an overview of the various characterization methods used to study poled glass structures.

\subsubsection{Etching Methods}

The most common technique of characterizing nonlinear materials is the Maker fringes method, described in detail in section 2.2.3. This method is very well suited for nonlinear crystals, where the thickness of the nonlinearity is much greater than 
the coherence length. The modulation fringes reveal information about the magnitude of the coherence length, and comparative measurements to a material with a known nonlinearity can be used in a fairly straightforward way to obtain the nonlinearity of the sample under test. However, in poled glasses, a typical thickness of the nonlinearity is $4-10 \mu \mathrm{m}$, which is less than the coherence length (at fundamental wavelength of $1064 \mathrm{~nm}$, the coherence length is $\sim 24 \mu \mathrm{m}$; at $800 \mathrm{~nm}$, it is $\sim 12 \mu \mathrm{m}$ ). In this case, the result of a Maker fringes measurement cannot be used to deduce the thickness of the nonlinear region, thus an independent measurement of the thickness is required. This can be done by etching the anodic surface of the glass in hydrofluoric (HF) acid in incremental steps, and remeasuring the signal after every etch [5]. If the nonlinearity is present only at the anode side, then the thickness of the depletion region corresponds to the etched depth at which the second harmonic signal becomes negligible.

The basic idea of HF etching to study the nonlinearity distribution was improved upon by taking advantage of the differential etch rates in the poled glass structures. It was shown in [92] that when silica glass is subjected to an electric field, the rate at which the glass etches in $\mathrm{HF}$ acid varies linearly with the field strength. An interferometric optical method was used to determine the thickness that was etched in real-time, and the period of oscillation of the interference trace was used to determine the etch rate. This method was then used to monitor the evolution of the depletion region in poled silica in real-time [93]. The experiment revealed that the electric field recorded in silica samples during the poling process is on the order of $10^{8} \mathrm{~V} / \mathrm{m}$, and the thickness of the depletion layer ranged between 5 and $20 \mu \mathrm{m}$, depending on the poling time.

The above technique was further improved by combining the interferometric measurement that determined the etched thickness with a simultaneous monitoring of the second harmonic signal generated in the sample as it was being etched [94]. The obtained information allows one to reconstruct the nonlinear spatial distribution created inside the glass. This technique, later named the layer peeling method, was used to study the time evolution of the nonlinear profiles induced within silica samples that were poled for different time durations [95]. It was shown that for poling durations 
shorter than 5 minutes the spatial distribution of the induced $\chi^{(2)}$ exhibits a triangular shape, and is in excellent agreement with charge migration models that involve a single charge carrier (such as mobile $\mathrm{Na}^{+}$impurity ions that migrate away from the anode). In contrast, for higher poling durations the nonlinear profiles tend to flatten, indicating that there is an additional charge carrier injected at the anode that plays an important role. A simulation that includes the mobilities of different mobile species was developed in [96] and was shown to agree very well with experiments of thermally poled Infrasil samples.

The layer peeling method is a powerful tool for characterizing the distribution of the nonlinearity in poled glass, although it is possible to speculate that the actual etching process may modify the distribution of the charges that are responsible for the induced SON. The main disadvantage of this method is that it is completely destructive, thus many researchers focused on developing different characterization methods that allow for non- or minimally-destructive measurements.

\subsubsection{Improved Maker Fringe Measurement Methods}

The main limitation of the standard Maker fringe method is the limited range of angles that can be used to probe the interior of the sample. As illustrated in Figure 3.1, refraction at the front air-sample interface and total internal reflection at the back of the sample-air interface limit the maximum attainable internal propagation angle within the sample to $\sim 43^{\circ}$ for silica. When the method is used to characterize a thin nonlinear region as is the case with poled glass, the pathlength within the nonlinear region is at most $L / \cos \theta_{\mathrm{TIR}}$, which is below the coherence length. Essentially the SH signal measured with standard Maker fringe measurement represents only a portion of the full data set (e.g. up to $\sim 43^{\circ}$ for silica), which is insufficient to determine the nonlinear coefficient and the depth of the nonlinear region.

One way to overcome this limitation of the standard Maker fringe method is to place the sample between two $45^{\circ}$ silica prisms as shown in Figure 3.1, a method known as prism-assisted Maker fringe measurement [58]. The front prism allows probing of the nonlinearity at large angles, while the back prism eliminates the total 


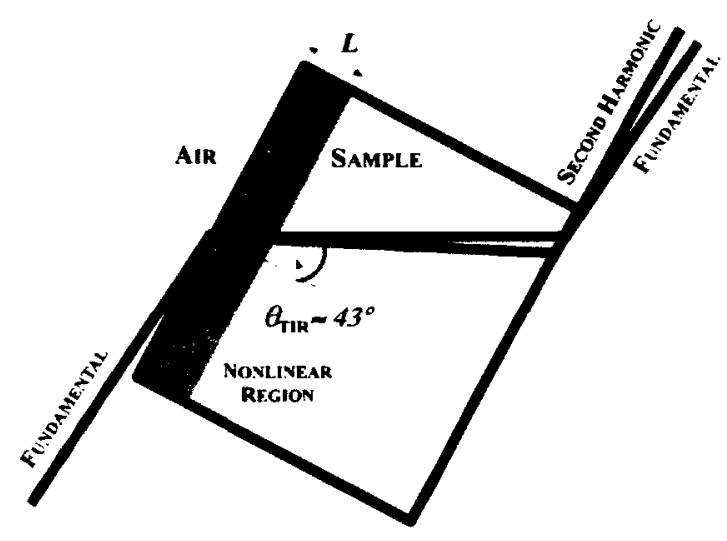

(a)

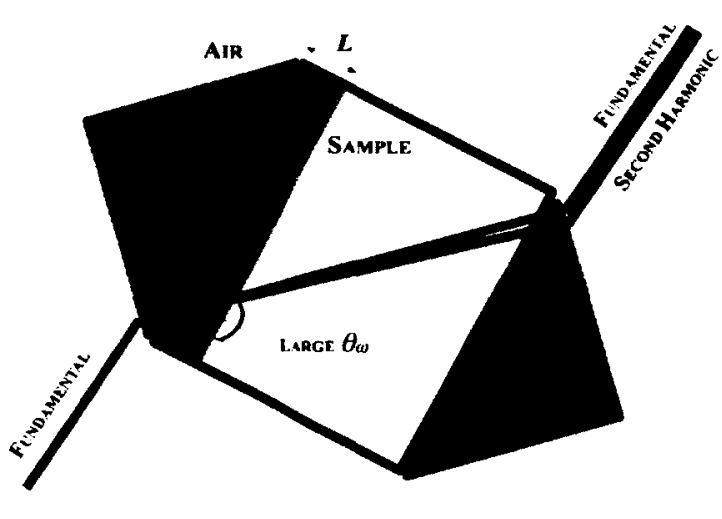

(b)

Figure 3.1: Maker fringe SHG measurement schemes on poled glass: (a) a standard Maker fringe setup, where total internal reflection limits the internal propagation angle ( $\sim 43^{\circ}$ for silica), (b) a prism-assisted Maker fringe setup, which increases the maximum propagation angle.

internal reflection at the back face of the sample. As a result, internal propagation angles up to $85^{\circ}$ can be reached, allowing a nearly full Maker fringe measurement for poled glass structures. The experimental data obtained from the prism-assisted Maker fringe measurement in [58] was fitted to the appropriate mathematical expression, and it was concluded that the nonlinear region in the poled Herasil silica sample has a Gaussian profile with a $1 / e$ width of $8 \mu \mathrm{m}$ and a maximum $\chi^{(2)}$ of $0.68 \mathrm{pm} / \mathrm{V}$. Improvements on the prism-assisted Maker fringe method include sphere-assisted Maker fringe technique, where a pair of hemispherical lenses is used on both sides of the sample [97] and cylinder-assisted Maker fringe technique, where cylindrical lenses are used [98]. The results obtained with these enhanced characterization methods highlight the importance of impurities in the poled glasses. While it was found in [58] that the nonlinear region spans $\sim 8 \mu \mathrm{m}$ in Herasil, and a similar result was obtained for Infrasil in [99], the results for poled Suprasil samples indicated that there exists a weak nonlinearity throughout the sample in addition to a nonlinearity peak at the anode, which spans $\sim 25 \mu \mathrm{m}$ [99]. The large disparity in the thickness of the induced nonlinear region was attributed to very different levels of impurities in these glasses - the sodium ion impurity concentration is at least 20 times higher in Infrasil 
and Herasil compared to Suprasil [100]. However, the reason for the existence of the bulk nonlinearity in Suprasil was not understood.

At least part of the reason why there might be such variations in the reported profiles of the induced nonlinearities is the way this information is inferred from the Maker fringe measurement data. In most of the above reports, the experimental data was fitted with a mathematical model with the implicit assumptions that the $\chi^{(2)}$ distribution has a single maximum and no change in its sign. However, this assumption might not be valid in poled glasses, especially in light of strong evidence for the existence of a second charge carrier near the anode during the poling process. Based on the mathematical theory of the Maker fringe method it is straightforward to show that because this method does not collect phase information, the solution for the $\chi^{(2)}$ distribution based on an experimental curve is not unique. As was shown in [101], $\chi^{(2)}$ profile with a single peak, two peaks, as well as two peaks with different signs can all generate essentially the same. experimental Maker fringe curve. Therefore, in order to find a unique solution for the nonlinearity distribution of a thin nonlinear region, the Maker fringe analysis must be used in conjunction with another independent measurement technique so that the general profile of the induced nonlinearity is known.

This limitation was partially overcome by a new Maker fringe method that involves stacking two identically poled samples together [102]. Assuming a step nonlinear profile within the samples, such a stacked measurement provides the same information as a standard Maker fringe measurement plus an etching measurement of the thickness of the nonlinear region. This method was enhanced in [103], where using the same stacked structure, an inverse Fourier transform technique was employed to uniquely determine both the shape and the location of the nonlinearity from the Maker fringe data. The resultant profile reveals spatial features that could not be resolved with previous techniques, and indicates that the depth of the nonlinearity is significantly deeper ( $\sim 25 \mu \mathrm{m}$ for a poled Infrasil sample) than was previously believed. Another variation of this methods uses a quartz plate stacked with the poled glass sample [104]. The addition of a plate with a known nonlinear distribution causes interference between the signals generated, allowing the phase of the measured signal to be accessi- 
ble, and enabling one to determine a complex spatial distribution of $\chi^{(2)}$ in the poled sample.

A very powerful method to retrieve a unique nonlinearity profile from Maker fringe measurement data was developed in [105]. The method uses an iterative loop known as the Fienup algorithm to process the data obtained from a cylinder-assisted Maker fringe measurement. Since only a single sample is needed (i.e. no stack is required), this is a completely non-destructive technique. Prism-, sphere- or cylinder-assisted Maker fringe measurements are required to obtain data for a larger set of internal propagation angles. This mathematical approach of retrieving the nonlinear profile works because a large number of practical poling profiles can be described by a family of functions known as minimum-phase functions, for which it is possible to recover Fourier transform phase from the Fourier transform magnitude alone.

Rather than trying to increase the pathlength of the beam above the coherence length, other researchers focused on decreasing the coherence length. This was accomplished in a non-collinear Maker fringe measurement reported in [106], where the second harmonic generated by two non-collinear fundamental beams is used. Compared to the standard one-beam case, this configuration reduces the coherence length of the process from $24 \mu \mathrm{m}$ to less than $2 \mu \mathrm{m}$ (for fundamental wavelength $\lambda=1.064 \mu \mathrm{m}$ ), thus increasing the resolution of the measurement. The sensitivity of this method can be further increased by varying the angle between the two fundamental beams, as was done in [67]. In both cases, rather than observing the envelope alone, clear modulation fringes can be seen, which can be used to deduce the depth of the nonlinear region.

\subsubsection{SHG Microscopy}

Another technique for measuring the second-order nonlinear coefficient in poled glass involves scanning a focused laser beam across the side of the sample, such that the beam propagates along the nonlinear region and perpendicular to the direction of the poling field. This technique is referred to as SHG microscopy, and it was first performed in [10], where the near-field pattern of the second harmonic was imaged by 
using a microscope objective and a video camera, and used to obtain the depth profile of the induced second-order nonlinearity. This technique was enhanced and used for studying the profile of the induced nonlinearity in thermally poled silica plates [107], silica-based waveguide structures [108] [109], and specialty poled fibers [74] [88]. Although second harmonic microscopy provides direct visualization of the sample's nonlinear profile, the preparation of the sample for the measurement can influence the field distribution within it. A sample is typically cleaved through the nonlinear region and side polished, which is likely to influence the distribution of the positive charges near the anodic surface, changing the field present in the sample and thus its nonlinearity profile.

\subsubsection{Other Methods}

A number of other methods were also used to study the charge distribution in poled glasses. Laser induced pressure pulse (LIPP) method is a non-destructive acoustic imaging technique, where a short duration laser pulse hits an absorbing layer attached to the sample, and creates a pressure wave that displaces the stored charge, creating a current signal. This technique was used to obtain information about the sign and distribution of charges in poled silica glass [60]. The results indicated that there are both positive and negative charge layers in the glass. A positive layer at the surface of the samples is believed to be caused either by emission of electrons from the glass to the electrode, or by attraction of positively charged ions from the air. A negatively charged layer, positioned deeper in the sample constitutes the depletion region caused by migration of cations away from the anode. The LIPP technique was combined with nuclear techniques for near-surface analysis in [110] to allow mapping of ions. It was determined that a sodium-depleted layer is always present after poling, and its thickness increases with the poling time. In some cases there is an accumulation of hydrogen near the anode, which is believed to come from residual water present on the surface of the sample.

Near-surface analysis of poled silica was also done by secondary ion mass spectroscopy (SIMS). The measurements in [111] indicate that sodium and lithium ions 
are depleted from a region spanning about $20 \mu \mathrm{m}$ near the anodic surface. In addition, potassium and sodium ions, and possibly some hydrogenated species are injected into the sample from surface contamination during poling. SIMS measurements of silica waveguides poled with silver electrodes show that high concentrations of silver are injected into the glass during the poling process [14] [112].

The electric field that is induced within the bulk of silica glass samples both during and after thermal poling treatment was studied in [113] by the electric field-induced second harmonic (EFISH) technique. While the setup could not scan the surface close to the anodic and cathodic surfaces, the measurements of the field distribution in the bulk of the sample indicated that during the poling process, the initially homogeneous electric field decays exponentially from the anodic to the cathodic surface. Moreover, after the poling treatment, a permanent electric field is induced within the bulk of the sample, and its profile also follows an exponential decay profile, a result that seems to disagree with the field distribution consistent with previously proposed models [64] [114]. The EFISH technique was also used to study the influence of surfaces and interfaces on the process of poling [115], where it was concluded that imperfections at internal and external interfaces within the poled structure caused unwanted exchange of charged species with the atmosphere, which was detrimental for the poling efficiency and stability.

Measurement of the electric surface potential using an earthed field meter is another method that was used to study the frozen-in electric field and the presence of charges in the nonlinear layer [116]. The results suggest that atmospheric particles deposit on both surfaces of the sample, acting as screening charges that cause the surface potential to go to zero. While the surface potential is zero, however, the SH signal generated in the sample remains constant, indicating that a voltage drop is still present within the sample, and allowing a measurement of the frozen-in electric field. 


\subsection{Glass Electro-Optic Modulators}

The linear electro-optic effect is proportional to the second-order nonlinear susceptibility, thus the encouraging results obtained with the SHG experiments in glasses suggested that efficient electro-optic devices could also be realized.

The first electro-optic phase modulator in poled silica was reported in [117]. A buried silica channel waveguide was formed by electron irradiation of bulk material. It was then thermally poled and inserted into an arm of a Mach-Zehnder interferometer to measure the phase shift in the poled phase modulator. The modulator produced a phase shift of $32 \mathrm{mrad}$ for $\lambda=633 \mathrm{~nm}$ for a device interaction length of $4.8 \mathrm{~mm}$ and an applied electric field of $7.3 \mathrm{~V} / \mu \mathrm{m}$. The relatively low value of the measured phase shift was thought to be due to the poor spatial overlap $(\sim 2 \%)$ between the nonlinear region and the waveguide mode. The poling-induced electro-optic coefficient in a similar experiment was estimated to be $r_{33}=0.3 \mathrm{pm} / \mathrm{V}$ [118]. A planar $2 \times 2$ switch was realized in [119] by constructing an integrated Mach-Zehnder interferometer with thermally poled $\mathrm{GeO}_{2}$-doped silica-based channel waveguides on a silicon substrate. The induced electro-optic coefficient was estimated to be $0.02 \mathrm{pm} / \mathrm{V}$, resulting in a switching voltage of $1700 \mathrm{~V}$ for $36 \mathrm{~cm}$-long phase-shifter, operating in the $1.55 \mu \mathrm{m}$ wavelength region. In [112], very large second harmonic signals were observed from silica waveguides poled with silver electrodes. However, when electro-optic measurements where done for the same samples with UV-written channel waveguides, only $r=0.05 \mathrm{pm} / \mathrm{V}$ like in previous experiments was achieved. It was suggested that the large SHG is related to injections of ions during poling, although SIMS concentration profiles did not reveal any simple consistent correlation between the SHG signal and the accumulation of any particular ionic species. It is also possible that the UV method of writing waveguides is ill-suited for poled glass structures, as there is experimental evidence that UV-exposure results in a significant reduction in the induced nonlinearity of thermally poled glasses [31].

The first thermally poled fiber phase modulator was reported in [120]. A germaniumdoped D-shaped fiber was used, and an electro-optic coefficient $r=0.05 \mathrm{pm} / \mathrm{V}$ was 
obtained. Later the poling process was optimized with a help of a polyimide layer, that served both as a bounding agent and as an insulating medium during the poling process, to result in a fabrication technique that is extensible to volume manufacturing with $r=0.3 \mathrm{pm} / \mathrm{V}$ [121]. Reducing the fiber thickness by mechanical polishing following the poling led to a half-wave drive voltage of only $75 \mathrm{~V}$ for a $12 \mathrm{~cm}$ long active device length [122]. A very high electro-optic coefficient of almost $6 \mathrm{pm} / \mathrm{V}$ was reported in [8] for germanosilicate fiber poled by the UV-poling method, but these results were not reproducible after the initial experiment. Poling of twin-hole germanosilicate fibers was studied extensively by an in situ thermal poling process described in [123]. The setup consisted of a free space Mach-Zehnder interferometer and allowed real-time monitoring of the evolution of the electro-optic coefficient during the poling process. The results support the frozen-in electrical field model, and suggest that thermal poling of silica fibers consists of two processes: an initial process of building up a shielding field and a subsequent process of establishing an ionization field. The lifetime of the nonlinearity in these poled germanosilicate fibers was estimated to be tens to a few hundreds of days [124] [86]. A specially designed, twin-hole fiber with a borosilicate layer on the inside of the anode hole was designed to extend the lifetime of the induced nonlinearity [125], where the borosilicate layer was believed to trap the negative charges created during poling. This work was one of the first reports to use the notion of charge-trapping to stabilize the poling-induced nonlinearity. An all-fiber interferometer setup designed in [126] was used for a multiwavelength interrogation of a twin-hole poled fiber, showing that the electro-optic response of the poled fiber reduced by $\sim 50 \%$ when the interrogation wavelength was changed from $633 \mathrm{~nm}$ to $1550 \mathrm{~nm}$.

A technological improvement of the twin-hole fiber poling process was suggested in [127] to address the difficulty in obtaining good contact between the electrodes and the walls of the holes. Molten alloy under high pressure was used to produce fibers with 1 meter long internal electrodes that are solid at room temperature. Although no poling was done in [127], but rather an intrinsic Kerr nonlinearity was exploited to realize electro-optic switching, this technology could be readily used in fiber poling experiments. This was done in [128], where a switching voltage of $1.37 \mathrm{kV}$ was 
achieved for a $20 \mathrm{~cm}$-long active fiber that acted as the active arm of a Mach-Zehnder interferometer, operating at $\lambda=1.55 \mu \mathrm{m}$. While the presence of the alloy electrodes introduced significant optical losses, it was believed that this technology could be used to significantly reduce the switching voltage by optimizing the poling process and increasing the length of the device to $\sim 1$ meter.

While the process of drawing of a liquid alloy through the capillary fiber holes can be used to realize fibers with internal electrodes on the order of a few meters, another method, which integrates the wire into the fiber during the drawing process, is scalable to kilometer lengths. This fabrication method was used in [129] to demonstrate a 200 meter-long optical fiber with an integrated electrode, exhibiting low optical losses at $1.55 \mu \mathrm{m}$. A 0.9 meter section of the fiber was thermally poled, successfully inducing a $\sim 0.0125 \mathrm{pm} / \mathrm{V}$ electro-optic coefficient, and higher values of the induced nonlinearity were predicted with an optimized fiber design.

Another interesting implementation of a poled glass electro-optic modulator was in a microstructured silica fiber, with a solid glass core and two electrode holes in the outer cladding [130]. $\sim 0.02 \mathrm{pm} / \mathrm{V}$ electro-optic coefficient at $\lambda=1.55 \mu \mathrm{m}$ was measured, comparable to that obtained with germanosilicate fibers. It was believed that the fiber geometry could be used to improve on this result.

\subsection{Poled Structures with Interfaces}

Most of the measurements of the induced nonlinearity in glasses reveal that the SHG active layers are very thin. This is especially true in structures that have interfaces, such as the interfaces between deposited thin films and a substrate. A few important experimental investigations have shown that the existence of interfaces has a pronounced effect on the induced nonlinearity during the poling process.

The first experimental evidence that highlighted the importance of interfaces was presented in [14], where it was shown that the SH signal peaked at interfaces between different glass layers in a thermally poled silica waveguide structure. Secondary ion mass spectrometry (SIMS) measurements of samples poled with silver electrodes revealed concentration peaks of sodium, potassium and silver occurring at the interfaces 
between pure silica and germanium-doped silicon oxy-nitride glass layers, and at the glass-silicon substrate interface [112].

A similar waveguide structure but with a PbO-silica core layer was studied in [15]. Assuming the second-order nonlinearity in glasses is induced through the frozenin field mechanism via $\chi_{\text {eff }}^{(2)}=3 \chi^{(3)} E_{d c}$, lead glass was used because its $\chi^{(3)}$ was about an order of magnitude higher than that of fused silica. The measurements indicated that the SHG efficiency peaked strongly on both sides of the lead-glass film, i.e. at the interfaces between the different glasses. It was concluded that strong $\left(\chi^{(2)} \sim 15 \mathrm{pm} / \mathrm{V}\right)$ nonlinearity was localized within the $200 \mathrm{~nm}$-thick lead glass layer, and its sign was opposite to that of the nonlinearity in silica.

The work in [16] looked at thermally poled D-shaped fibers with germaniumdoped core. Here it has been clearly shown that the core-cladding interface impedes the migration of charges that are believed to be responsible for induced SON in poled glasses. The effect of this interface barrier depends on the electric field established in the structure during the poling process, and can significantly influence the evolution of the SON in the structure [131]. A similar effect was also observed in planar structures, where it was concluded that germanosilicate films act as a barrier for migration of cations during poling [132] and for diffusion of positive ions such as $\mathrm{H}_{3} \mathrm{O}^{+}$from the anode surface into the sample [13]. The strong effect of glass interfaces was even more pronounced in [133], where larger second-order nonlinearities were found at the interfaces between different germanium-doped layers.

\subsection{Poled Multilayered Structures - Thesis Objectives and Expectations}

If so much research has been done in the area of poled glasses, why is silica not used as a nonlinear material in today's optical devices? Although much effort has been invested into optimizing the poling process and exploring different variations of poling, so far the nonlinear interactions in resulting devices have remained too weak to be used for practical applications. This can be attributed to three main problems. 
The first problem is the relatively low value of the induced nonlinear susceptibility coefficient, which has not been significantly improved upon over the initial result of $\chi^{(2)} \sim 1 \mathrm{pm} / \mathrm{V}$. Although this value is quite far from the strongest tensor element of lithium niobate $\left(\chi_{33}^{(2)} \sim 81 \mathrm{pm} / \mathrm{V}\right)$, it is comparable to the $\chi^{(2)}$ values of other nonlinear crystals that are widely used for frequency doubling. So, in fact, the current value of $1 \mathrm{pm} / \mathrm{V}$ is adequate for many practical applications, especially considering the long interaction lengths that are possible with silica waveguide- or fiber-based devices. A second and more severe problem with poled glass devices is the difficulty in obtaining high nonlinear susceptibility over thicknesses compatible with standard waveguide or fiber dimensions. In glasses that have a relatively high impurity content (e.g. Infrasil), where $\chi^{(2)} \sim 1 \mathrm{pm} / \mathrm{V}$ values are routinely obtained, the induced nonlinear region is only a few micrometers thick [5]. This results in a poor overlap between the nonlinearity and the optical field propagating through the glass, and thus inefficient nonlinear interaction [117]. On the other hand, glasses where the impurity content is much lower (e.g. Suprasil), do exhibit poling-induced nonlinear regions that span tens of micrometers, but the magnitude of the induced nonlinear effect is about an order of magnitude lower than in the glasses with higher impurity content [5]. This problem cannot be overcome by poling for longer durations, as detailed experiments on poling conditions showed that a competing detrimental process decreases the poling efficiency for long poling times [96]. This process involves injection of positive charges from the anode, which substitute for the impurity alkali ions that have migrated away, thus reducing the established electric field and decreasing the induced nonlinear effect. The third problem has importance from a practical reproducibility standpoint. The impurity levels in bulk silica glasses are typically on the order of a few ppm, and can vary greatly between ingots of nominally identical glass. Because the dynamics of the poling process strongly depend on the impurity content, it is difficult to control the extent and the magnitude of the induced nonlinearity [134].

The main hypothesis behind this doctoral project is that the second problem of overlap can be solved by poling multilayered glass structures with several layers distributed over large thicknesses. As is evident from the reports quoted in section 3.4, nonlinearity peaks tend to be located near interfaces. In this work this idea is taken 
further to design a structure that is specifically engineered to take advantage of the unique nonlinear effects near interfaces. Thus even if the maximum possible nonlinear coefficient in each layer is limited to $\sim 1 \mathrm{pm} / \mathrm{V}$ by fundamental physical constraints, both SHG and electro-optic modulation of light will increase because of the larger cumulative thickness over which the SON occurs. The approach taken in this project is to study silica glass layers doped with various dopants as building blocks for the design of a structure that allows for an enhanced effective SON upon poling. Just as germanium-doped layers have been shown to act as barriers for migration of charges, it is reasonable to assume that while some dopants act as "charge-blocking" layers, other dopants may be used to create efficient "charge-carrying" layers, allowing or even facilitating migration of charges. If variously doped glass layers can be used to control the distribution and the migration of the mobile carriers that are believed to be responsible for the creation on the nonlinearity, then it is conceivable that a properly designed multilayered structure can act as multiple ionic charge accumulating capacitors in series. This idea is consistent with an ionic RC circuit model for the dynamics of a thermal poling process, where the induced nonlinear region behaves like a nonlinear capacitor [135] [136]. The multilayered design extends this idea to a multi-capacitor scheme, where the applied poling voltage may be divided between the different layers, thus poling multiple layers simultaneously. Moreover, unlike the flame hydrolysis process that is used to manufacture synthetic silica glass, where the levels of impurities may vary significantly between different ingots [134], standard thin film deposition processes (such as plasma-enhanced chemical vapor deposition) tend to be more controllable. Therefore, the amount of impurities that participate in the poling process in the multilayered structures is expected to be consistent between devices fabricated at different times, resulting in an improved reproducibility, and allowing the possibility of a controlled introduction of impurities into the multilayered structure as a means of enhancing the induced nonlinearity.

The previous two paragraphs identified the main issues with poled bulk glasses, and showed the promise of the multilayered design. If indeed the multilayered silicabased design is successful, meaning that it is able to realize a strong second-order nonlinear region that spans over thicknesses compatible with standard waveguide or 
fiber dimensions, what applications can be realistically benefited? First, let us consider arguably the most demanding application for second-order nonlinear materials high-speed electro-optic modulation. Would an electro-optic modulator based on the silica-based multilayered design be able to offer serious competition to lithium niobate, the de facto nonlinear material for today's multi- $\mathrm{GHz}$ modulation systems? A silica-based modulator would have the benefits of monolithic integration, no insertion losses or thermal mismatch problems, and would eliminate the need for pigtailing and packaging, but to be a viable replacement for lithium niobate it would also have to take not much more physical space, and be able to modulate the optical signal at very demanding rates $(40 \mathrm{~Gb} / \mathrm{s}$ and higher). Consider the standard equation for the electro-optic phase accumulated over length $L$ (previously derived as part of equation (2.62)):

$$
\Delta \phi=\frac{2 \pi}{\lambda} n^{3} r \frac{V}{2 d} L \Gamma
$$

assuming standard parameter values $(d=10 \mu \mathrm{m}, V=5 \mathrm{~V}, r=0.3 \mathrm{pm} / \mathrm{V}, \lambda=1.55 \mu \mathrm{m})$ and a perfect overlap $(\Gamma=1)$, we get that in order to achieve $\pi$ phase shift, $L=3.5 \mathrm{~m}$ is required. Typical dimensions of a $40 \mathrm{~Gb} / \mathrm{s}$ lithium niobate modulator are $0.4 \times 7 \mathrm{~cm}$ (or an area of $2.8 \mathrm{~cm}^{2}$ ). By taking some standard waveguide parameter that are used it today's planar lightwave circuit (PLC) systems $(3 \times 3 \mu \mathrm{m}$ waveguides, $30 \mu \mathrm{m}$ interwaveguide spacing, $1 \mathrm{~mm}$ minimum radius of curvature) and assuming a dense doublecoiled waveguide design [137], it can be calculated that an approximately $7.7 \mathrm{~m}$-long waveguide can fit within the area equivalent of a lithium niobate modulator. Now, if a Mach-Zehnder interferometer configuration is used for intensity modulation, two arms will need to fit within this area, so that the length of the active region is reduced to $\sim 3.8 \mathrm{~m}$, which is more than enough to accommodate the $3.5 \mathrm{~m}$ required to obtain $\pi$ phase shift.

But there is another factor that determines the maximum length of the electrooptic modulator. As was discussed in section 2.3, in order to achieve high-speed modulation typically a traveling-wave electrode configuration is used. In this configuration the maximum modulation speed is limited by the phase mismatch between the optical and the RF modulating wave. Since the dispersion of lithium niobate is rela- 
tively high ( $n=2.2$ at $1.55 \mu \mathrm{m}$ but approaches $n=6$ at microwave frequencies [138]), the bandwidth-interaction length product for lithium niobate electro-optic modulators is limited to $\sim 9 \mathrm{GHz}-\mathrm{cm}$ [45] (although advanced velocity-matching schemes can be used to increase this product). In this respect the low dispersion of silica glass is an advantage, however it is not realistic to expect that even with the lower dispersion an interaction length of a few meters can be achieved without the two waves slipping out of phase. Moreover, the loss in the metal electrodes at microwave or mm-wave frequencies causes a decay of the modulating field, and in practice limits the effective length of the transmission lines to a few centimeters at the most [47]. Therefore, unless there is a significant increase in the nonlinear coefficient of poled glass devices (which is not the primary goal of this work, although some progress towards this will be discussed in the body of this thesis), it is unlikely that poled glass devices can meet the strict demands of today's high-speed electro-optic modulators.

However, there are many other nonlinear applications where poled multilayered silica-based structures may in fact prove to be a more flexible, inexpensive and problem-free alternative to existing technologies. First, consider their use as electrooptic switches. PLC systems often employ multi-material systems to achieve lowspeed switching capabilities (e.g. polymer thermo-optic switches). Such multi-material systems are undesirable for many reasons - additional fabrication steps, thermal and optical mismatches, etc. The introduction of a silica-based electro-optic switch that operates at hundreds of $\mathrm{MHz}$ speeds would have a tremendous impact in terms of ease of integration and a decrease in the power consumption of the switching module. Because such a switching-fabric does not have the requirement of very high modulation speed, the limitations on the construction of the electrodes are relaxed, and assuming the same parameter values as indicated above, a $\pi$ phase shift can be realized within an area of $2.5 \mathrm{~cm}^{2}$ (assuming the conservative electro-optic coefficient value $r=0.3 \mathrm{pm} / \mathrm{V}$ and a perfect overlap).

Second, consider the area of frequency doubling. Crystalline materials such as quartz, KDP (potassium dihydrogen phosphate) or KTP (potassium titanyl phosphate) are routinely used for doubling the frequency of high-intensity ultrashort pulses and their nonlinear coefficients are on the order of $1 \mathrm{pm} / \mathrm{V}$. If we can realize a suf- 
ficiently thick silica-based nonlinear material such that a high overlap is possible, such artificially-created nonlinear material would undoubtedly be superior to these crystalline materials (in terms of the cost, the reflection losses, etc.).

Finally, we can speculate that the idea of a multilayered silica-based nonlinear metamaterial could be expanded to silica fibers (although this is beyond the scope of this thesis). A preform consisting of concentric layers of silica can be prepared by MCVD (modified chemical vapor deposition, similar to the fabrication of Bragg microstructured optical fibers [139]), and periodic poling can be realized using one of the approaches described in sections 3.1 and 3.3. Such nonlinear silica-based fibers can be directly spliced with rare-earth doped lasers to realize stable and rugged sources in the visible spectrum. Moreover, they can serve as a second-order nonlinear medium for spontaneous parametric down-conversion for the generation of photon pairs in optical cryptography applications. 


\section{Chapter 4}

\section{Sample Fabrication and Characterization Methods}

This chapter presents the experimental methods used to fabricate, pole, and characterize the multilayered samples studied in this project. The main goal of this research has been to realize a second-order nonlinear material that is maximally compatible with current optical material systems. Silica glass meets this requirement as the most prevalent material in optical fibers and planar lightwave circuits. In order to retain the compatibility, the multilayered structures explored in this project are restricted to commonly used silica glass dopants and standard microfabrication techniques. Section 4.1 describes the two thin film deposition methods used to fabricate the multilayered structures - low pressure chemical vapor deposition, and plasmaenhanced chemical vapor deposition. The motivation behind the two poling techniques chosen to induce second-order nonlinear effects in the multilayered samples is the topic of section 4.2. Lastly, the second harmonic generation scheme used to characterize the induced nonlinearity is described in section 4.3. 


\subsection{Thin Film Deposition}

The first set of samples were deposited by low pressure chemical vapor deposition (LPCVD) at the microfabrication facilities at Carleton University. The pre-deposition cleaning process involved the first two steps of the standard RCA clean process: (1) removal of organic contaminants by a solution of ammonium hydroxide, hydrogen peroxide and water at $80^{\circ} \mathrm{C}$; and (2) removal of ionic contaminants using a solution of hydrogen chloride, hydrogen peroxide and water at $80^{\circ} \mathrm{C}$. Undoped, phosphorusdoped and boron-doped silica layers were deposited by LPCVD at $420^{\circ} \mathrm{C}$ using the precursor gases shown in Table 4.1. The samples did not undergo a post-deposition annealing / densification step. The substrates used for all LPCVD-deposited samples were double-side polished ES-grade synthetic fused silica by Tosoh Quartz [140] (nominal thickness $500 \mu \mathrm{m}$ ) cut from the same ingot to ensure identical impurity content. Based on the gas flow rates during deposition, the phosphorus and boron content for the LPCVD doped layers is estimated to be $6 \mathrm{w} \%$ and $4 \mathrm{w} \%$, respectively. Because of the vertical positioning of the substrates in the LPCVD chamber, equivalent stacks of layers were deposited on both sides of the substrates. These LPCVD samples were used in the corona poling experiments presented in Chapter 5.

\begin{tabular}{lll}
\hline Deposited Film Type & LPCVD Chemistry & PECVD Chemistry \\
\hline Undoped silica & $\mathrm{SiH}_{4}, \mathrm{O}_{2}$ & $\mathrm{SiH}_{4}, \mathrm{~N}_{2} \mathrm{O}$ \\
Phosphorus-doped silica & $\mathrm{SiH}_{4}, \mathrm{O}_{2}, \mathrm{PH}_{3}$ & $\mathrm{SiH}_{4}, \mathrm{~N}_{2} \mathrm{O}, \mathrm{PH}_{3}$ \\
Boron-doped silica & $\mathrm{SiH}_{4}, \mathrm{O}_{2}, \mathrm{~B}\left(\mathrm{OCH}_{3}\right)_{3}$ & - \\
Germanium-doped silica & - & $\mathrm{SiH}_{4}, \mathrm{~N}_{2} \mathrm{O}, \mathrm{GeH}_{4}$ \\
\hline
\end{tabular}

Table 4.1: Precursor gases used for the deposition of silica-based multilayered structures by LPCVD and PECVD.

The second set of samples were deposited by plasma-enhanced chemical vapor deposition (PECVD) at the microfabrication facilities at Communications Research Centre Canada. The pre-deposition cleaning process involved: (1) removal of organic contaminants by RR2 resist remover at $85^{\circ} \mathrm{C}$; and (2) removal of adventitious 
deposits using a boil in acetone followed by a boil in isopropyl alcohol. Undoped, phosphorus-doped and germanium-doped silica layers were deposited at $300^{\circ} \mathrm{C}$ using the precursor gases shown in Table 4.1. The substrates used for all PECVD-deposited samples were double-side polished Suprasil 300 synthetic fused silica by Heraeus [100] (nominal thickness $630 \mu \mathrm{m}$ ) cut from the same ingot to ensure identical impurity content. Some of the samples were annealed at $950^{\circ} \mathrm{C}$ for 4 hours in nitrogen to study the effect of annealing on the induced nonlinearity. EDX analysis performed on deposited layers showed that the PECVD system is capable of depositing layers with $0 \cdots 7.6 \mathrm{w} \%$ phosphorus content, and 0-22.5 w\% germanium content. Unlike the LPCVD case, the substrates are positioned horizontally in the PECVD chamber, therefore the multilayered stacks are deposited only on one side of the substrates. The PECVD samples were used for the thermal poling experiments described in Chapter 6.

The choice of the deposition methods (LPCVD and PECVD) was not done due to physical reasons, but due to the availability of different fabrication facilities as the project progressed. Tables 4.2, 4.3 and 4.4 summarize the samples investigated in the course of this project, and point to the appropriate pages in the thesis where their characterization is presented.

\begin{tabular}{|c|c|c|c|c|c|}
\hline & $\begin{array}{c}\text { SAMPLE } \\
\text { NAME } \\
\end{array}$ & $\begin{array}{l}\text { DEPOSITION } \\
\text { METHOD }\end{array}$ & Deposited Structure & $\begin{array}{c}\text { STACK } \\
\text { ThickNesS } \\
\end{array}$ & Results \\
\hline \multirow{4}{*}{ 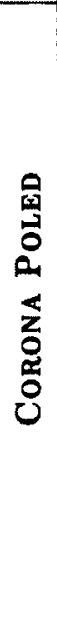 } & $B$ & LPCVD & Tosoh bulk glass substrate & $0 \mu \mathrm{m}$ & p. $75-78$ \\
\hline & $\mathrm{BU}$ & LPCVD & $\begin{aligned} & 2 \text { layers: } \text { undoped silica }(1 \mu \mathrm{m}) \\
& \text { B-doped silica }(0.2 \mu \mathrm{m}, 4 \mathrm{w} \%) \\
& \text { (stacks on both sides, Tosoh substrate) }\end{aligned}$ & $1.2 \mu \mathrm{m}$ & p. $79-80$ \\
\hline & $\mathrm{PU}$ & LPCVD & $\begin{aligned} & 2 \text { layers: } \text { undoped silica }(1 \mu \mathrm{m}) \\
& \text { P-doped silica }(0.2 \mu \mathrm{m}, 6 \mathrm{w} \%) \\
& \text { (stacks on both sides, Tosoh substrate) }\end{aligned}$ & $1.2 \mu \mathrm{m}$ & p. $79-80$ \\
\hline & PUPU & LPCVD & $\begin{aligned} & 4 \text { layers: } \text { undoped silica }(1 \mu \mathrm{m}) \\
& \text { P-doped silica }(0.2 \mu \mathrm{m}, 6 \mathrm{w} \%) \\
& \text { undoped silica }(1 \mu \mathrm{m}) \\
& \text { P-doped silica }(0.2 \mu \mathrm{m}, 6 \mathrm{w} \%) \\
& \text { (stacks on both sides, Tosoh substrate) }\end{aligned}$ & $2.4 \mu \mathrm{m}$ & p. $79-84$ \\
\hline
\end{tabular}

Table 4.2: Summary of corona poled samples investigated in this thesis. 


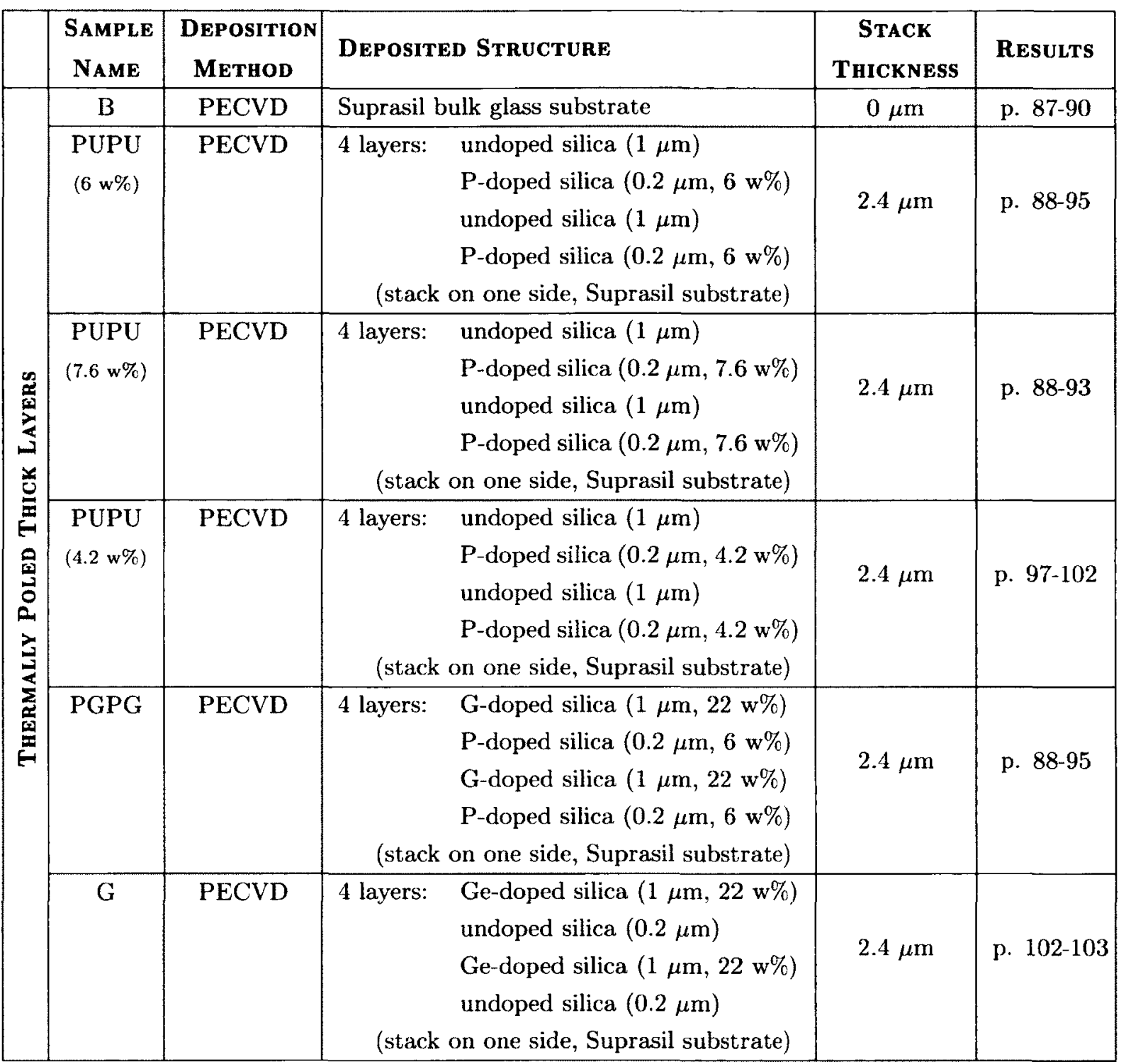

Table 4.3: Summary of thermally poled samples with thick layers investigated in this thesis.

\subsection{Poling Methods}

As described in the literature review in Chapter 3, many different poling techniques have been developed with varying degrees of success. As of today, however, thermal poling appears the most reliable technique to induce second-order nonlinearity in silica glass. None of the other poling methods were able to consistently 


\begin{tabular}{|c|c|c|c|c|c|}
\hline & $\begin{array}{c}\text { SAMPLE } \\
\text { NAME }\end{array}$ & $\begin{array}{c}\text { Deposition } \\
\text { METHOD }\end{array}$ & Deposited Structure & $\begin{array}{c}\text { StaCk } \\
\text { Thickness }\end{array}$ & Results \\
\hline \multirow{4}{*}{ 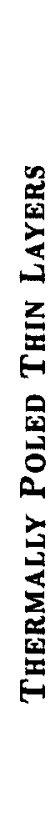 } & $\begin{array}{c}\mathrm{M} \\
(1.5 \mu \mathrm{m})\end{array}$ & PECVD & $\begin{array}{l}20 \text { alternating layers: } \\
\text { undoped silica }(75 \mathrm{~nm}) \\
\text { Ge-doped silica }(75 \mathrm{~nm}, 0.2 \mathrm{w} \%) \\
\text { (stack on one side, Suprasil substrate) }\end{array}$ & $1.5 \mu \mathrm{m}$ & p. 105 \\
\hline & $\begin{array}{c}\mathrm{M} \\
(3 \mu \mathrm{m})\end{array}$ & PECVD & $\begin{array}{l}40 \text { alternating layers: } \\
\text { undoped silica (75 nm) } \\
\text { Ge-doped silica ( } 75 \mathrm{~nm}, 0.2 \mathrm{w} \%) \\
\text { (stack on one side, Suprasil substrate) }\end{array}$ & $3 \mu \mathrm{m}$ & p. $102-107$ \\
\hline & $\begin{array}{c}\mathrm{M} \\
(4 \mu \mathrm{m})\end{array}$ & PECVD & $\begin{array}{l}54 \text { alternating layers: } \\
\text { undoped silica (75 nm) } \\
\text { Ge-doped silica ( } 75 \mathrm{~nm}, 0.2 \mathrm{w} \%) \\
\text { (stack on one side, Suprasil substrate) }\end{array}$ & $4 \mu \mathrm{m}$ & p. 105 \\
\hline & $\begin{array}{c}M \\
(5 \mu \mathrm{m})\end{array}$ & PECVD & $\begin{array}{l}67 \text { alternating layers: } \\
\text { undoped silica }(75 \mathrm{~nm}) \\
\text { Ge-doped silica }(75 \mathrm{~nm}, 0.2 \mathrm{w} \%) \\
\text { (stack on one side, Suprasil substrate) }\end{array}$ & $5 \mu \mathrm{m}$ & p. 105 \\
\hline
\end{tabular}

Table 4.4: Summary of thermally poled samples with thin layers investigated in this thesis.

induce higher nonlinearities, and their principle of operation is far less known compared to thermal poling. Therefore, thermal poling was an obvious first choice for our work on poled multilayered structures. As illustrated in Figure 4.1(a), a sample was sandwiched between poling electrodes, and placed on an electric hotplate within an enclosed chamber. The electrode stack consisted of pressed-on, highly-doped n-type silicon pieces (approximate size $1 \times 1 \mathrm{~cm}$ ) positioned with the polished sides in contact with the sample, and stainless steel electrodes $(1.2 \mathrm{~cm}$ in diameter) pressed against the silicon electrodes. The choice of electrode configuration was based on a previously reported observation of a stronger $\chi^{(2)}$ coefficient induced with pressed-on n-type silicon electrodes compared to p-type silicon or deposited metal electrodes [141]. The pre-poling cleaning process of the sample and the two silicon electrodes included: (1) a 10 minute rinse in de-ionized water, and (2) a 10 minute rinse in isopropyl alcohol, both done in an ultrasonic bath. A thermocouple probe was used to monitor the temperature of the top electrode. When the sample reached $300^{\circ} \mathrm{C}$, it was kept at 
this temperature for 30 minutes before applying the poling voltage such that the temperature was stable and homogeneously distributed. The chamber was continuously purged with nitrogen for the duration of the poling process to ensure a reproducible atmosphere. A poling voltage of $3 \mathrm{kV}$ was applied across the sample for the duration of 8 minutes, following which the hotplate was turned off allowing the sample to cool to room temperature over the course of $\sim 1.5$ hours, and the voltage was switched off. In all thermal poling experiments the samples were poled with the layered side oriented towards the anode.

In addition to thermal poling, another poling method is particularly interesting in the context of multilayered design. Corona poling uses a similar configuration to thermal poling, except the anode electrode is replaced with a needle held above the sample. As will be discussed in detail in Chapter 5, silica glass multilayers may be used to realize a structure that behaves as a series of charge-accumulating capacitors. Such a structure may overcome the limitations encountered in corona poling of bulk glass and thus can possibly result in a stronger cumulative nonlinearity. Figure 4.1(b) illustrates the corona poling setup used for our experiments. A tungsten needle, held at $8 \mathrm{~mm}$ above the sample, constituted the anode electrode. The ground electrode stack consisted of a pressed-on, highly-doped n-type silicon piece (approximate size $2.5 \times 2.5 \mathrm{~cm})$ positioned with the polished side in contact with the backside of the sample, and a stainless steel electrode $(2.5 \times 2.5 \mathrm{~cm})$ pressed against the silicon. The poling voltage set up between the needle and the ground electrode ranged from 6.4 to $8.6 \mathrm{kV}$. The sample cleaning process, the poling timeline and the nitrogen-rich atmosphere were identical to those used for the thermal poling experiments.

Much research has been done on the optimization of the poling conditions of bulk silica glass, with the conclusions often varying between different reports (e.g. [5], [142], [143]). The poling conditions described above are roughly based on previous reports, but are not optimized for our samples because the investigation of the effect of poling conditions on the induced nonlinearity is not the main goal of this project. It is highly likely that the optimal poling conditions for multilayered structures and for bulk glass are very different. However, the main goal of this project is to study whether the multilayered design is a viable tool in enhancing the induced second- 


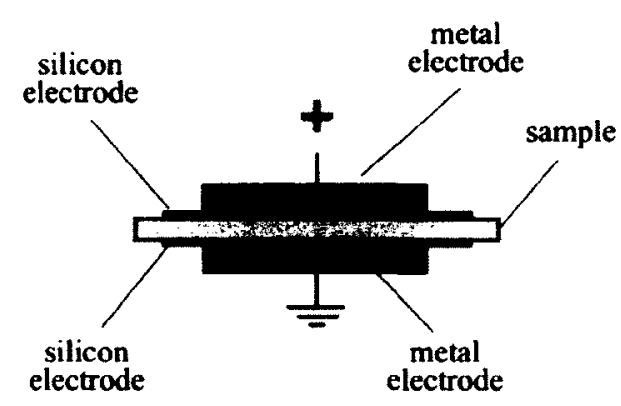

(a)

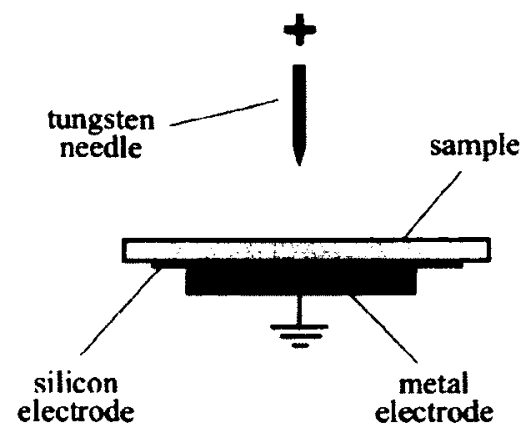

(b)

Figure 4.1: Poling methods used in this project: (a) a thermal poling setup, (b) a corona poling setup.

order nonlinearities in silica. Therefore, we have decided to look at the effects of layer composition, configuration, thicknesses, and so on, but the poling conditions remained constant throughout the project for both the bulk glass and the multilayered samples. The study of the effect of poling voltage, temperature, atmosphere, timeline, and electrode materials on the induced nonlinearity in multilayered structures would be an interesting project onto itself, and would undoubtedly provide more insight into the benefits and challenges of the multilayered design.

\subsection{Nonlinear Characterization Setup}

The poling-induced nonlinear properties in our samples are evaluated by measuring the second harmonic generated (SHG) in the samples using the Maker fringe method [36]. The SHG setup constructed for this purpose is shown in Figure 4.2. The laser source is a mode-locked Ti:sapphire laser operating at a wavelength of $800 \mathrm{~nm}$, pulse width $<70$ fsec, $80 \mathrm{MHz}$ repetition rate, $>125 \mathrm{~kW}$ peak power, and $>900 \mathrm{~mW}$ average power. Although an ultrafast laser is not an ideal choice for SHG measurements on poled glasses for reasons discussed later in this section, this has been the only pulsed laser source with sufficiently high average power specifications available for this project. A horizontally-polarized laser beam is amplitude-modulated by an optical chopper operating at $750 \mathrm{~Hz}$ in order to increase the signal-to-noise ratio 
of the detected second harmonic signal. The light is focused on a sample using a lens ( $75 \mathrm{~mm}$ focal length), and is recollimated using an additional lens (100 $\mathrm{mm}$ focal length). The focused beam radius is approximately $100 \mu \mathrm{m}$, and the average intensity incident on the sample is estimated to be $800 \mathrm{~W} / \mathrm{cm}^{2}$. A half-wave plate is used to rotate the polarization of the fundamental light, and the polarizer is used as an analyzer to detect only a specific polarization of the second harmonic light. A high-pass filter at the output of the laser filters out any light below $700 \mathrm{~nm}$. As the spectral response of the photomultiplier (Hamamatsu H7827-001 $19 \mathrm{~mm}$ diameter voltagetype photomultiplier tube) is in the $300-650 \mathrm{~nm}$ range, it is shielded from any stray light using a custom enclosure, and two filters at the input of the photomultiplier are used to remove the intense fundamental beam, so that only the second harmonic is detected. The signal from the photomultiplier serves as an input to the lock-in amplifier, with the chopper output port serving as a reference signal. The control of the rotation stage and data acquisition from the lock-in amplifier is done using a Labview program. The sample is positioned on a three-axis linear stage and an automated rotation stage. The rotation stage is aligned such that its rotation centre is located precisely at the beam waist by observing the intensity and the symmetry of the Maker fringe measurement for a quartz crystal. A typical measurement run involves adjusting the position of the sample such that it is positioned at the beam waist, and recording the generated SH signal as a function of the rotation angle.

The use of a femtosecond laser source for SHG presents some difficulties. Typical Maker fringe SHG setups use a Nd:YAG laser source operating at $1.064 \mu \mathrm{m}$ with pulse durations ranging from $40 \mathrm{psec}$ [83] to $200 \mathrm{nsec}$ [104]. The spatial extent of such pulses is $8 \mathrm{~mm}$ to 40 meters. The theory of the Maker fringe SHG measurements, as presented in section 2.2.3, applies to the continuous wave case. Since the spatial extent of the above pulses is far larger than the typical sample thickness used $(\sim 1 \mathrm{~mm})$, these cases can be considered quasi-cw, and thus the theoretical description in section 2.2.3 can be used. The evaluation of the induced nonlinear coefficient can be done in a fairly straightforward way by comparing the SH signal generated in the sample to that of a known standard, and using equation (2.34).

However, in the case of a $70 \mathrm{fsec}$ pulse, the spatial extent is only $14 \mu \mathrm{m}$, comparable 


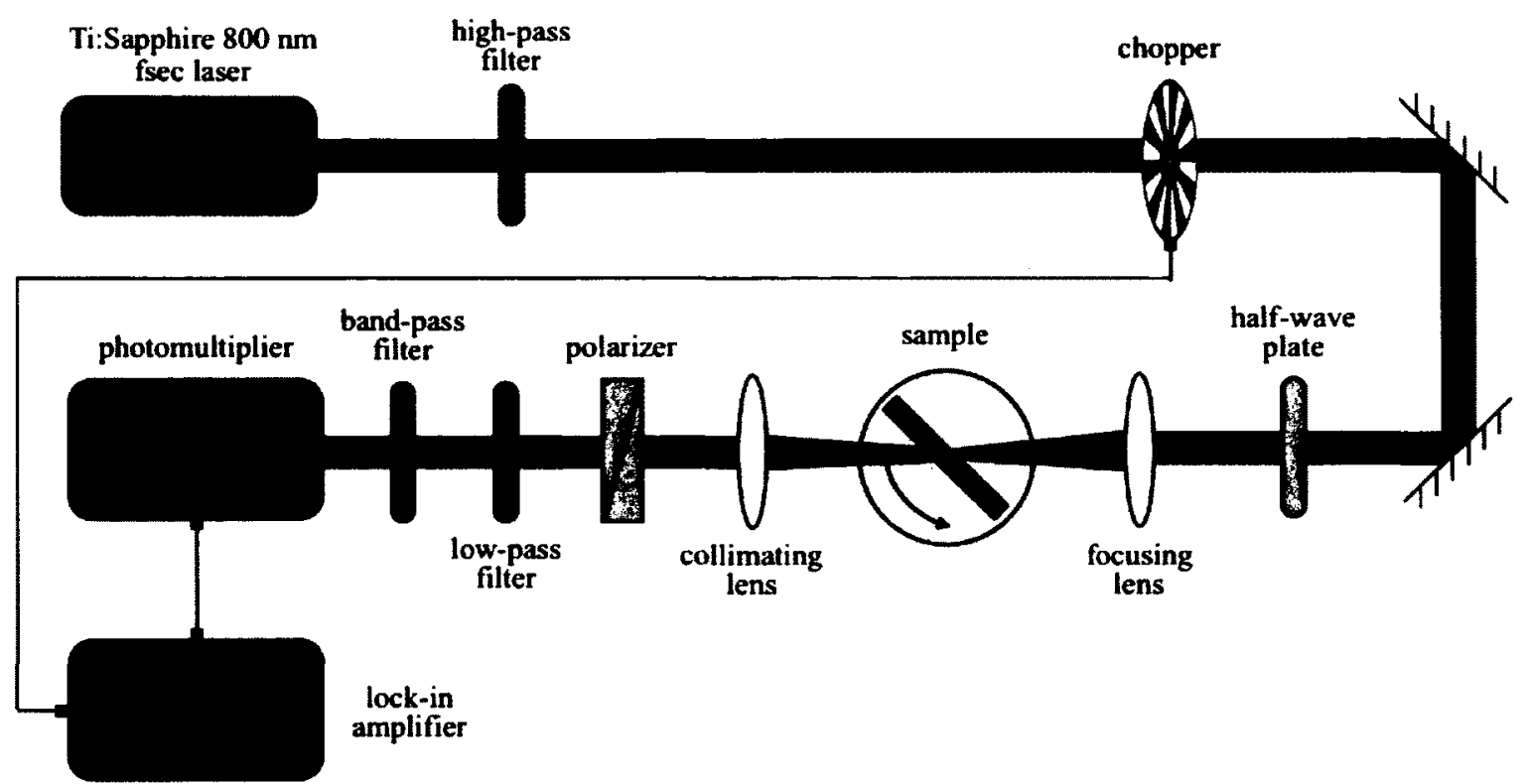

Figure 4.2: Maker fringe SHG measurement setup used for the nonlinear characterization of the poled samples.

to the thickness of the induced nonlinear region in poled silica and far less than the typical sample thickness. Therefore, the quasi-cw approximation no longer holds, and pulse propagation effects must be taken into account. The bound and the free second harmonic pulses now travel with different group velocities, and after a while they no longer physically overlap. This group velocity mismatch damps the Maker fringes, especially for thick samples or materials with high dispersion [144]. Unlike in the quasi-cw case, where Maker fringe measurements of a known standard result in well defined fringes that can then be used to find an angle at which the nonlinear interaction length is equivalent to a single coherence length, in the ultrafast case fringes of a thick crystal are not visible. Therefore, comparative measurements are no longer straightforward, and can only be done if ultrafast effects are included in the derivation of the Maker fringe theory. It was previously shown in Figure 2.8 that interference between two thin nonlinear regions results in modulation fringes, and the depth of the fringes can be used to obtain information about the relative magnitude of the two nonlinearities. In the ultrafast Maker fringe measurement, however, the second harmonic pulses generated in the two regions do not physically 
overlap (or overlap only partially). As a result, the total second harmonic signal detected consists only of the sum of the intensities, and fringes are either damped (in the case of partial overlap) or are completely washed out (in the case of total separation).

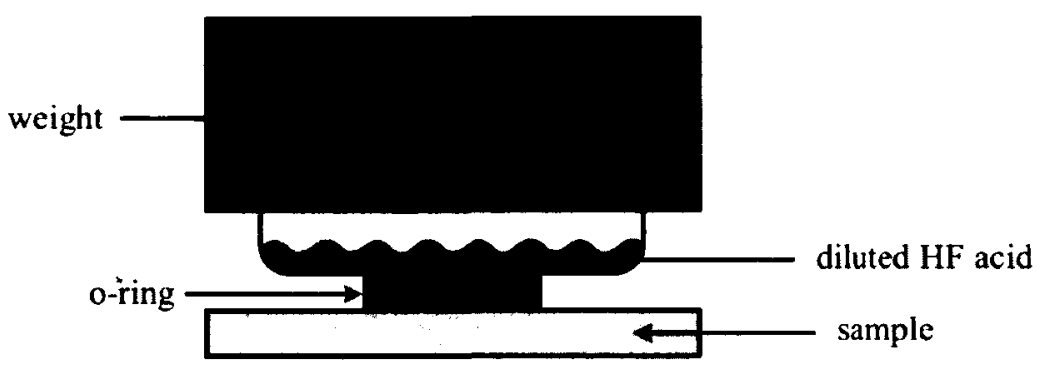

Figure 4.3: Hydrofluoric acid localized etching setup.

Despite these limitations of the Maker fringe measurements using an ultrafast laser source, the setup shown in Figure 4.2 is adequate for this project. The relative magnitude of the induced nonlinearity in different samples can be determined from the intensity of the Maker fringe envelope. However, Maker fringe measurement itself cannot be used to uniquely determine the profile of the induced nonlinearity [101], therefore our SHG measurements were combined with etching experiments to obtain information about the $\chi^{(2)}$ distribution. The approach taken in this project was to perform a series of localized etches on the poled samples in dilute hydrofluoric (HF) acid, and compare the SHG in the unetched and etched areas. The localized etches were accomplished by constructing the setup shown in Figure 4.3, where a vessel containing $\mathrm{HF}$ was pressed against the sample, and an o-ring was used to confine the etching solution to a small area. Dilute HF was used to avoid the introduction of significant surface roughness [5]. The etched depth was measured by a Dektak stylus surface profiling system. 


\section{Chapter 5}

\section{Investigation of Corona Poled Multilayered Structures}

Corona poling is a commonly used method for inducing second-order nonlinear properties in polymer films, where it results in an orientation of organic dye molecules in a host polymer [145]. The high poling field is produced by the ionized charges deposited on the sample surface through a corona discharge process. In the context of glasses, corona poling experiments reported to date resulted in nonlinear coefficient values up to $0.4 \mathrm{pm} / \mathrm{V}[146][80]$ and attributed the induced effect to migration of mobile ions [81]. It is generally believed that similar physical mechanisms are responsible for the induced nonlinearity both in the case of thermal and corona poling. As described in Chapter 3, during poling a frozen-in electric field is created as a result of a negatively charged depletion region and positive charges injected from the anode. This electric field couples with the intrinsic third-order nonlinearity of the glass and results in an effective second-order nonlinearity. In the case of thermal poling, the positive charges injected from the anode have a limited effect on the strength of the induced nonlinearity, at least for short poling durations [96]. In contrast, when corona poling of bulk glasses was studied in [83] it was concluded that because the anodic face of the sample is exposed to the atmosphere, a continuous injection of positive charges from the anode surface destroyed the formation of a depletion region, thus 
inducing only a weak bulk nonlinear effect. It is possible that this interpretation is not entirely correct, since the mobility of the injected positive charges is much lower than that of the alkali impurities [96], thus a depletion region should still form. A more plausible reason for the absence of a depletion region in [83] is that the surface potential that results from the corona discharge, whose value is likely to be more than an order of magnitude lower than the potential applied to the needle electrode [147], may be below the voltage threshold required to create a second-order nonlinear susceptibility by charge migration [62]. Nonetheless, since the main goal of this project is to use silica-based layers to control the distribution and the movement of charges, an interesting question arose as to whether multilayers can be designed to realize a structure that behaves like a series of charge-accumulating capacitors? In such a structure, each sub- $\mu \mathrm{m}$-thick capacitor would require a lower poling voltage to form a localized depletion region, resulting in a stronger cumulative nonlinear effect to be induced using the corona poling method. This higher degree of control over charge migration that may be possible with multilayered glass structures motivated the corona poling work as part of this thesis.

This chapter begins with a description of corona poling experiments on bulk glass (section 5.1). Section 5.2 presents the multilayered structures and the experiments designed to study the effect of glass film composition on the induced nonlinear effect upon corona poling. Section 5.3 summarizes the results of this chapter, and discusses their significance in the context of practical second-order nonlinear glass structures.

\subsection{Corona Poled Bulk Glass}

As described in Chapter 4 , in this project the poling-induced nonlinearity was characterized by comparative SHG measurements in combination with etching experiments. To establish a baseline for comparison, the first corona poling experiment involved poling of bulk silica glass - a synthetic fused silica substrate (Tosoh Quartz ES-grade; see sample B in Figure 5.1), identical to the substrates used for film deposition in the following sections. The poling conditions were described in detail in section 4.2 and remained constant for all the samples discussed in this chapter. 


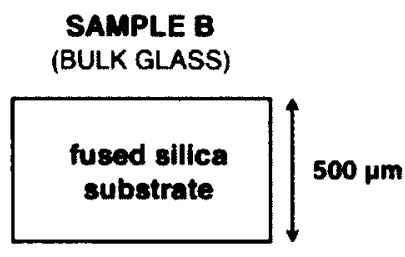

(a)

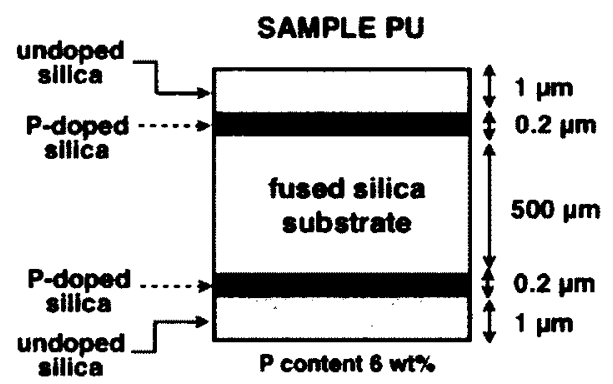

(c)

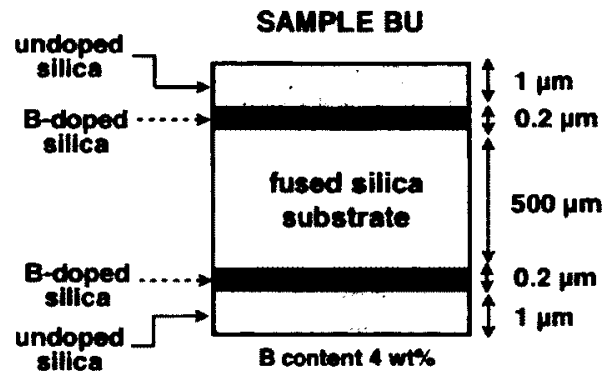

(b)

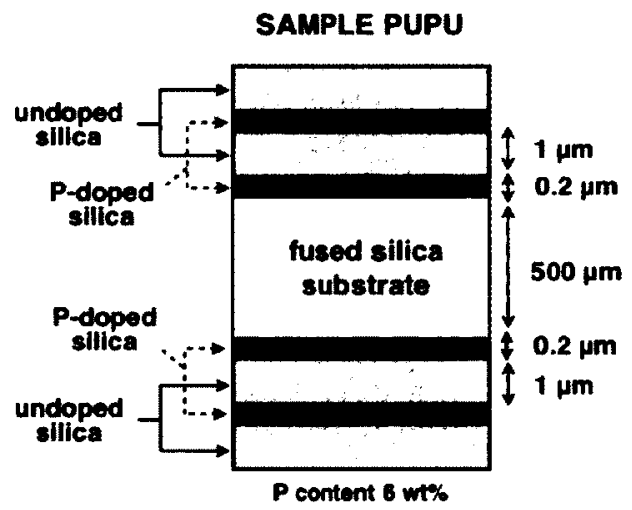

(d)

Figure 5.1: Corona poled silica glass structures: (a) a bulk glass sample, (b) a twolayered stack of undoped and boron-doped layers, (c) a two-layered stack of undoped and phosphorus-doped layers, (d) a four-layered stack of alternating undoped and phosphorus-doped layers.

Figure 5.2 shows the second harmonic signal generated in sample B. To determine the distribution of the nonlinearity, localized areas of the poled sample were etched using the setup described in section 4.3, and the remaining SHG was remeasured after every etch. The following observations and conclusions can be made based on the results presented in Figure 5.2:

1. Within the experimental error, when the sample is etched from the anodic side, the SHG remains constant for etches deeper than $17 \mu \mathrm{m}$, indicating that the sample exhibits a $\sim 17 \mu$ m-thick $\chi^{(2)}$ peak near the anodic surface. 


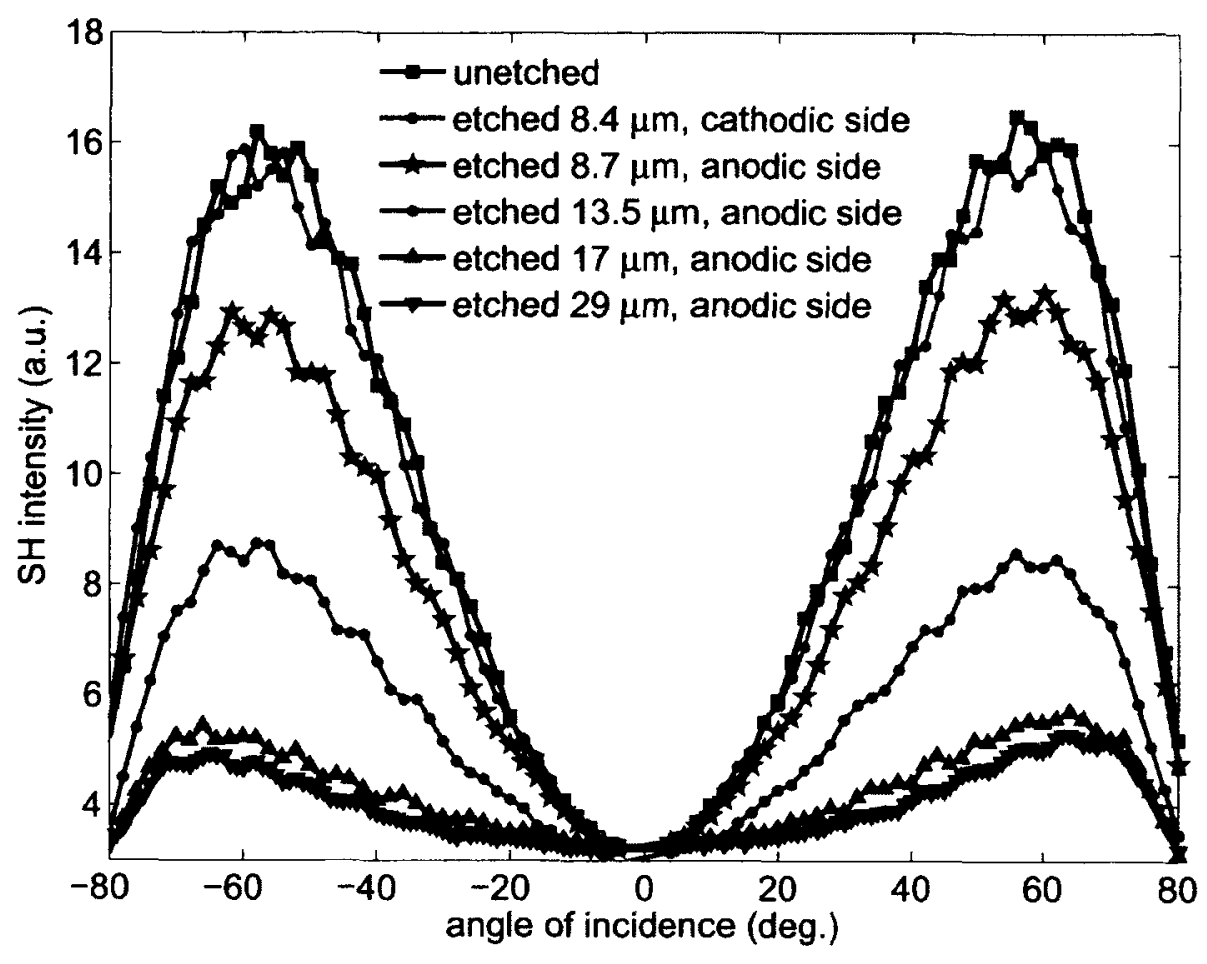

Figure 5.2: Experimentally measured SHG in corona poled bulk glass (sample B) as the sample is etched.

2. No change in the SHG Maker fringe envelope is observed when the sample is etched from the cathodic side, indicating that there is no nonlinearity peak near the cathodic surface.

3. The presence of modulation fringes and a non-zero second harmonic signal when the anodic nonlinearity peak is etched away indicate that a weak nonlinearity extends throughout the bulk of the sample.

These observations are consistent with previous reports of corona poled bulk glass (e.g. [83]). The fact that the modulation fringes are the result of bulk nonlinearity can be used to verify the theoretically derived value of the coherence length in fused silica. Recall that the fringes in the Maker fringe measurements result from the $\sin ^{2}(\Delta k L / 2)$ term in the equation for the second harmonic intensity (see equation (2.12)). The zeros (or, in the case of ultrafast SHG, the minima) occur when this term goes to 
zero, i.e.:

$$
m \pi=\frac{\Delta k L}{2}=\frac{1}{2} \frac{\pi}{L_{c}} \frac{L}{\cos \vartheta}
$$

where $m$ is an integer and equation (2.14) was used. While the value of $m$ is unknown, two adjacent minima are given by $m$ and $m+1$, resulting in the following two expressions for the $m^{\text {th }}$ and $m^{\text {th }}+1$ minima in the Maker fringe waveform:

$$
\begin{aligned}
m \pi & =\frac{\pi}{2} \frac{L}{L_{c}} \frac{1}{\cos \vartheta_{m}} \\
(m+1) \pi & =\frac{\pi}{2} \frac{L}{L_{c}} \frac{1}{\cos \vartheta_{m+1}}
\end{aligned}
$$

Subtracting these two expressions yields:

$$
L_{c}=\frac{L}{2}\left[\frac{1}{\cos \vartheta_{m+1}}-\frac{1}{\cos \vartheta_{m}}\right]
$$

Here $\vartheta_{m}$ and $\vartheta_{m+1}$ refer to the internal propagation angles that result in the $m^{\text {th }}$ and $m^{\text {th }}+1$ minima (and are related to the external propagation angle $\theta$ through equation (2.23)). Although the non-zero minima in ultrafast SHG introduce an error in the above calculation [21], an average value of $12.3 \mu \mathrm{m}$ coherence length is obtained for the experimental data in Figure 5.2, in reasonable agreement with $11.9 \mu \mathrm{m}$ calculated using equation (2.16) based on the Sellmeier equation for fused silica.

\subsection{Corona Poled Multilayered Structures}

The multilayered silica structures used in the corona poling experiments are shown in Figure 5.1. As described in Chapter 4, the thin film silica films were fabricated by LPCVD, where the vertical positioning of the substrates in the deposition chamber resulted in equivalent stacks of layers on both sides of the substrate.

Three sets of experiments were designed to study the corona poling-induced nonlinearity in these structures. The goals of the first set of experiments were:

1. To determine whether poling of multilayered silica-based structures increases the induced nonlinearity compared to the nonlinearity induced in poled bulk silica glass. 
2. To study whether the choice of glass dopants (phosphorus or boron) has an effect on the induced nonlinearity.

Following the corona poling treatment, the samples illustrated in Figure 5.1 resulted in the second harmonic measurements shown in Figure 5.3. The measurements show that the second harmonic (and thus the induced effective nonlinearity) is always higher in the multilayered structures than in bulk silica. While the increase is modest for the structure with boron-doped layers (sample BU), there is a 14-fold enhancement in the SHG in structures with phosphorus doping (samples PU and PUPU). Note that the SHG in the multilayered structure that did not undergo poling is insignificant, thus the enhanced nonlinearity can be attributed to the effect of the multilayered structures on the poling process.

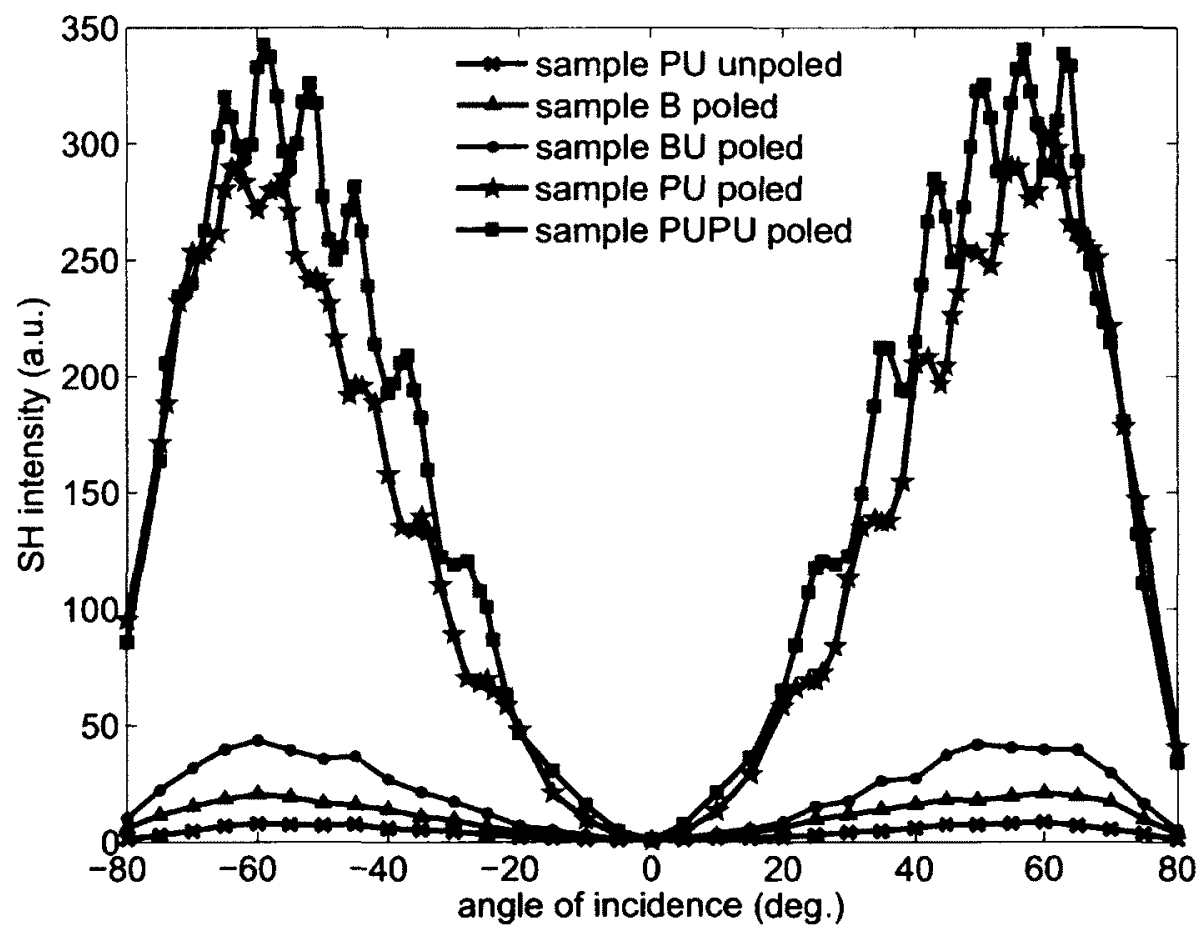

Figure 5.3: Experimentally measured SHG in corona poled multilayered structures shown in Figure 5.1.

A comparison of the poled multilayered structures in Figure 5.3 indicates that the doping of the layers is crucial to achieving a significant enhancement in the induced 
nonlinearity. The effectiveness of the samples with phosphorus-doped layer is believed to be related to the way phosphorus incorporates into the silicon dioxide network. Both phosphorus and boron substitute for $\mathrm{Si}^{4+}$ in the $\mathrm{SiO}_{4}$ tetrahedra, and form oxides themselves (primarily $\mathrm{P}_{2} \mathrm{O}_{5}$ and $\mathrm{B}_{2} \mathrm{O}_{3}$, respectively) [148]. However, because phosphorus has five valence electrons (compared to four in silicon), non-bridging oxygen ions become associated with the phosphorus sites. These oxygens have local negative charges, which trap any positive ions drifting through the lattice through a coulombic interaction. This property has been used extensively to limit migration of sodium impurity ions to stabilize the behavior of early MOSFET devices [149]. Unlike phosphorus-doped silica, which essentially forms a trapping layer for positive ions, boron is incorporated into the glass network in a trivalent form, thus creating electron-deficient centers that have no affinity for positive ions.

Based on the encouraging results with phosphorus-doped layers, the rest of the corona poling experiments dealt exclusively with four-layered structures identical to sample PUPU. In the second set of experiments, the dependency of the induced nonlinearity on the poling voltage was studied, with the summary of the results presented in Figure 5.4. A higher poling voltage results in an increased nonlinearity, and the SHG in the poled multilayered samples is consistently about an order of magnitude higher than in bulk silica glass poled under the same conditions.

The goal of the third set of experiments was to study the distribution of the nonlinearity in the multilayered structures. In the case of bulk glass poling, as positive impurity ions move away from the anode, the applied voltage drops primarily across the established depletion region. However, as shown above, the use of doped silica layers allows a greater control over the migration of charges during the poling process. This observation gives rise to an intriguing idea - can the layers be designed such that the entire structure behaves as a few charge-accumulating capacitors [135] in series? If this is the case, then the applied voltage could be divided between the "capacitors" such that a few regions of glass are poled simultaneously. Since the samples studied in this chapter have multilayered stacks on both sides of the substrate, the merit of this idea can be tested by investigating whether the poling-induced nonlinearity is concentrated only near the anode side of the sample (as in the bulk glass case), or 


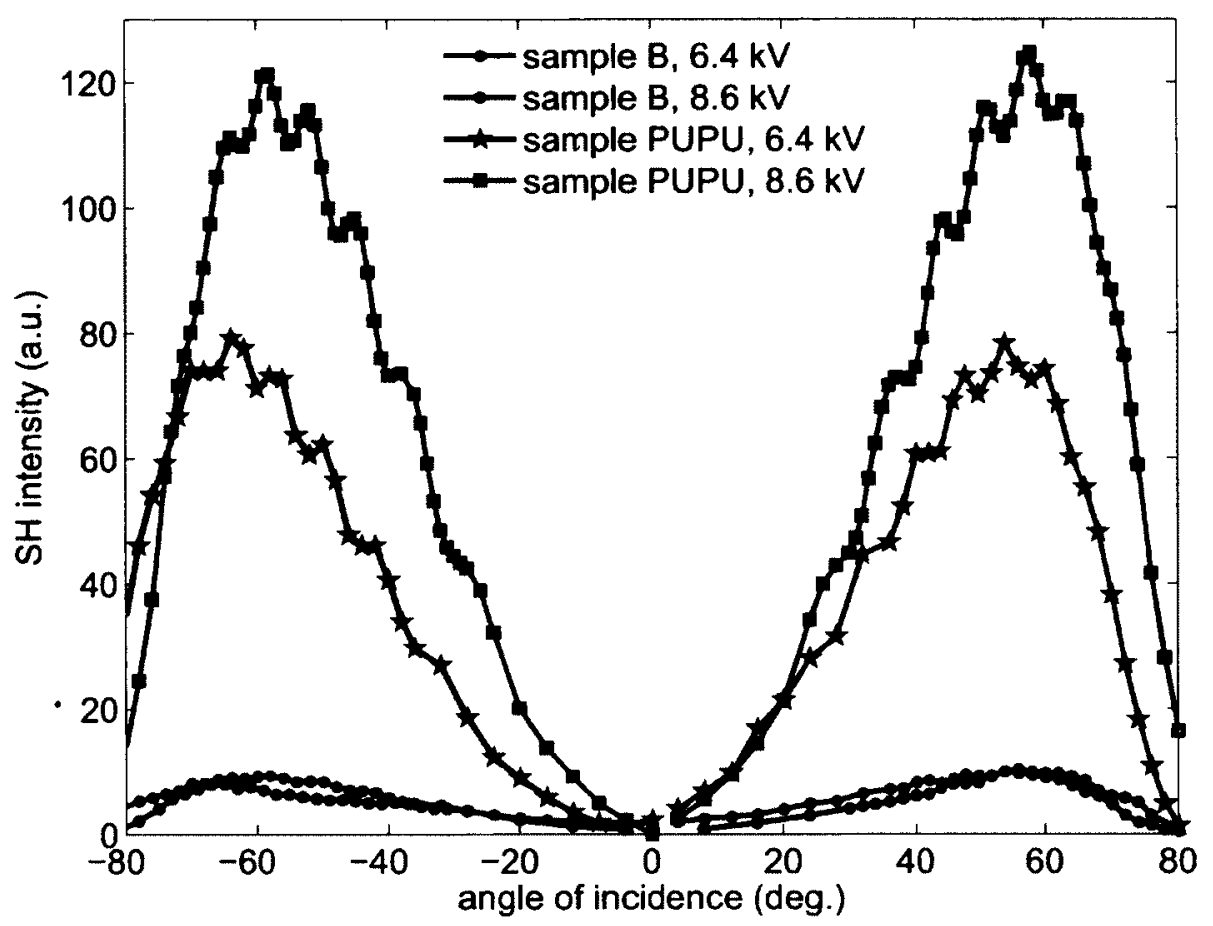

Figure 5.4: Experimentally measured SHG in corona poled bulk silica glass (sample B) and multilayered sample PUPU for different poling voltages.

is divided between the two multilayered stacks (which would indicate simultaneous poling of the two regions).

To study the distribution of the nonlinearity, localized regions on the poled multilayered sample were etched in dilute hydrofluoric acid (using the setup described in section 4.3), and the SHG in the remaining thickness was measured. As can be seen in Figure 5.5, when the anodic side of the poled sample is etched, the SH signal gradually decreases. This trend stops at etch depths greater than $10 \mu \mathrm{m}$, at which point the SH signal remains the same (within the experimental error). When the same experiment was repeated on the cathodic side of the sample, a very similar behavior was observed (see Figure 5.6). This leads to the conclusion that comparable nonlinearities were induced by poling on both sides of the sample (both spanning between 6 and $10 \mu \mathrm{m}$ ). Note that in a typical Maker fringe experiment, two comparable nonlinearities on both sides of the sample would result in deep $(\sim 100 \%)$ modulation fringes. In this case, however, the fringes are severely damped due to the ultrafast laser source that 
is used for the second harmonic generation (see section 4.3). Most importantly, the presence of two nonlinear regions on both sides of the sample indicates that the poling voltage was divided between the two multilayered stacks, realizing simultaneous poling of multiple glass regions.

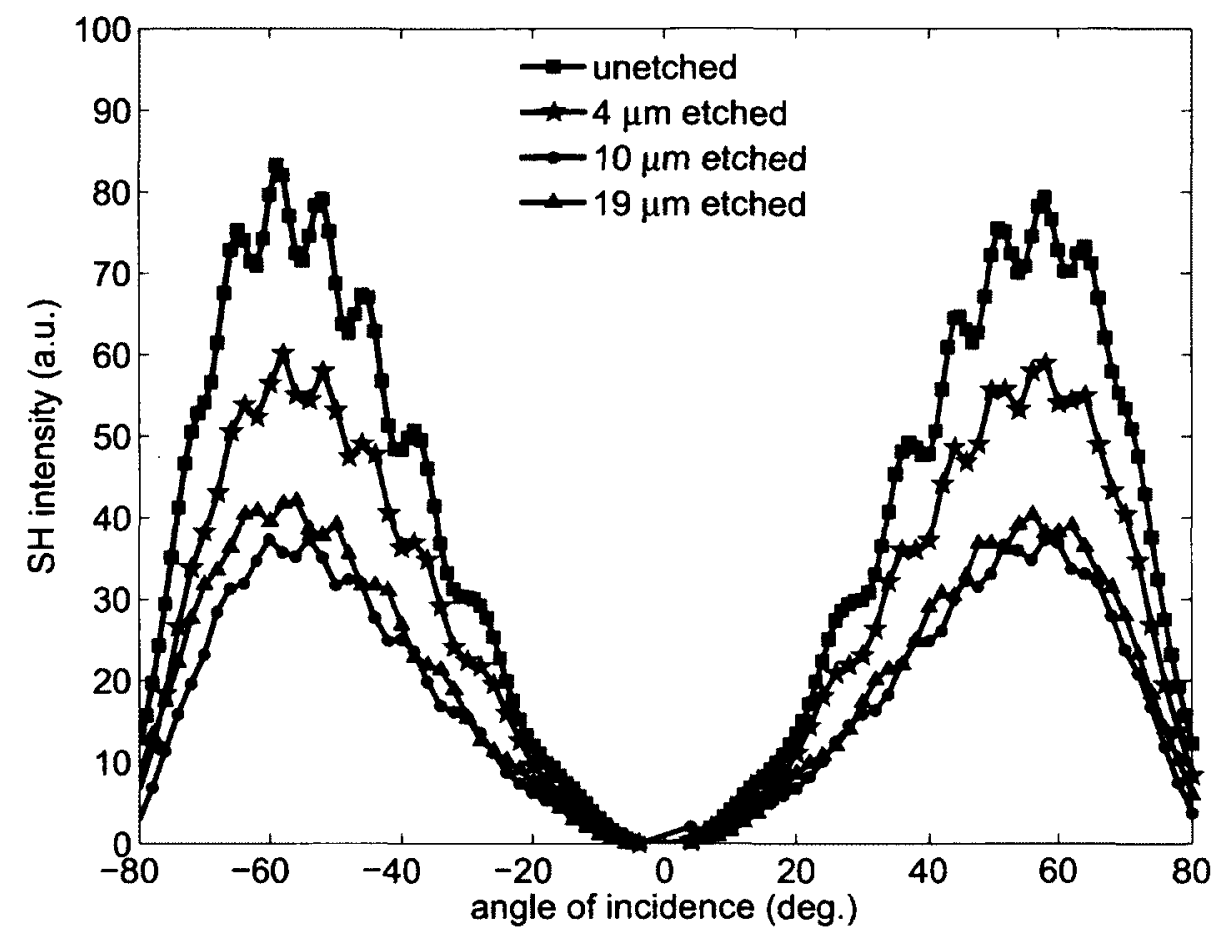

Figure 5.5: Experimentally measured SHG in corona poled multilayered sample PUPU as the anodic side is etched.

In the case of bulk glass, previous studies reported no nonlinearity peak at the cathode [99], or estimated it to be extremely thin [72]. In the case of sample PUPU, however, the nonlinearity at the cathode is comparable in magnitude and thickness to the nonlinearity induced at the anode side of the sample. In order to verify that this is a consequence of the multilayered design and not of our specific poling process, the SHG in structure PUPU as shown in Figure 5.1 ("double-stack sample") was compared to the SHG in an identical structure but with the backside layers removed prior to poling ("single-stack sample" PUPU'). Unlike in the double-stack sample case, the Maker fringe measurement for the single-stack sample did not exhibit any modulation fringes (see Figure 5.7), indicating that the remaining nonlinearity extends over 


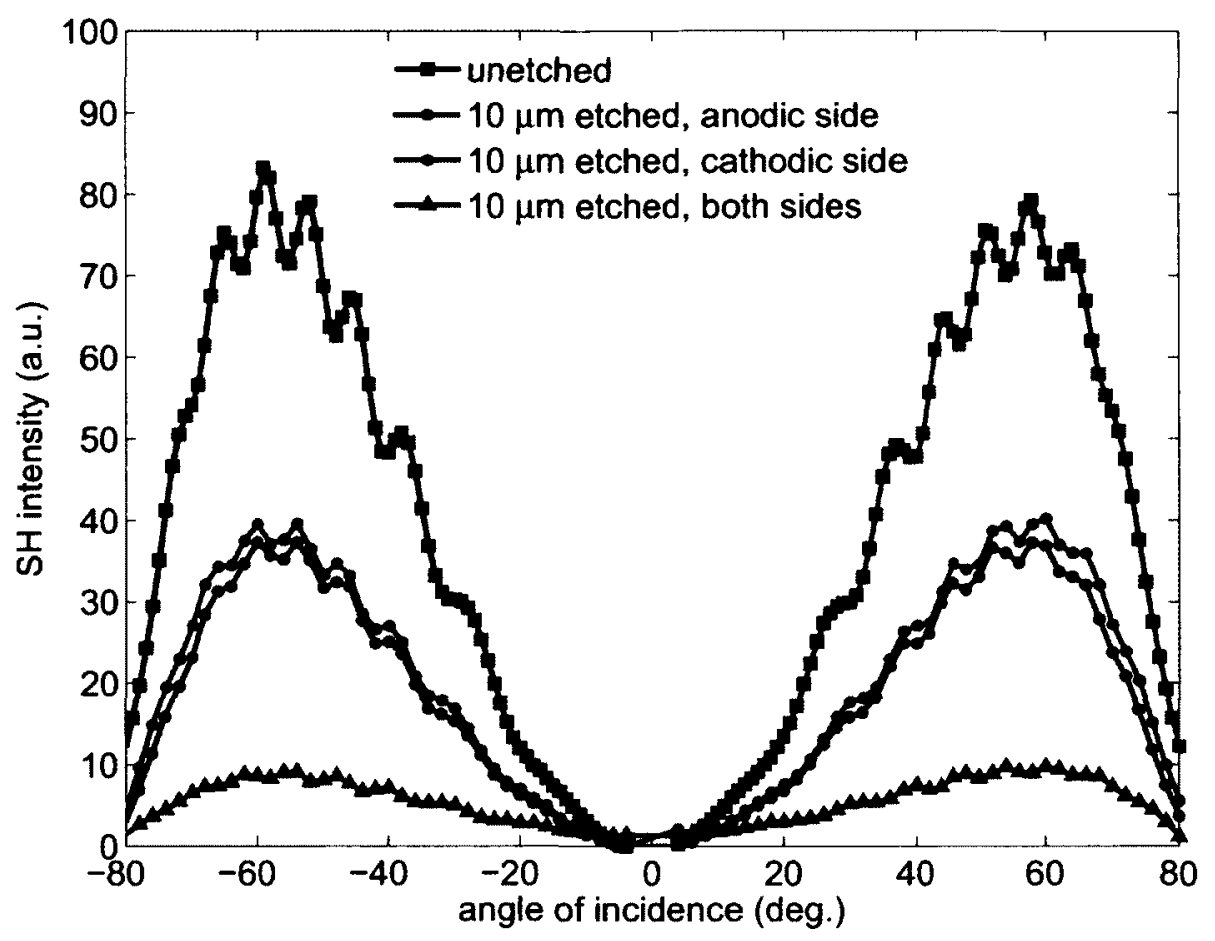

Figure 5.6: Experimentally measured SHG in corona poled multilayered sample PUPU as both sides of the sample are etched.

a region shorter than the coherence length in silica $(11.9 \mu \mathrm{m}$ at $800 \mathrm{~nm})$. When the cathodic side of the poled single-stack sample was etched, no change in the SH was observed. This confirms that the single-stack sample has only a single nonlinearity peak at the anode, and thus the cathodic nonlinearity peak in the double-stack sample is a direct result of the backside multilayers.

The model presented earlier, whereby the higher nonlinearity in sample PUPU is attributed to the ability of phosphorus-doped silica to trap positive charges, can explain the nonlinear peaks that are formed on both sides of the samples. First, consider the anodic side - in the case of bulk glass, the depletion region formed during the poling process is diminished by the injection of detrimental positive charges from the anodic surface. The addition of phosphorus-doped layers traps these charges, thus preventing them from propagating further into the sample and affecting the depletion region. It is also possible that the phosphorus-doped layers cause accumulation of alkali impurity ions in their vicinity, thereby creating strong localized nonlinearity 


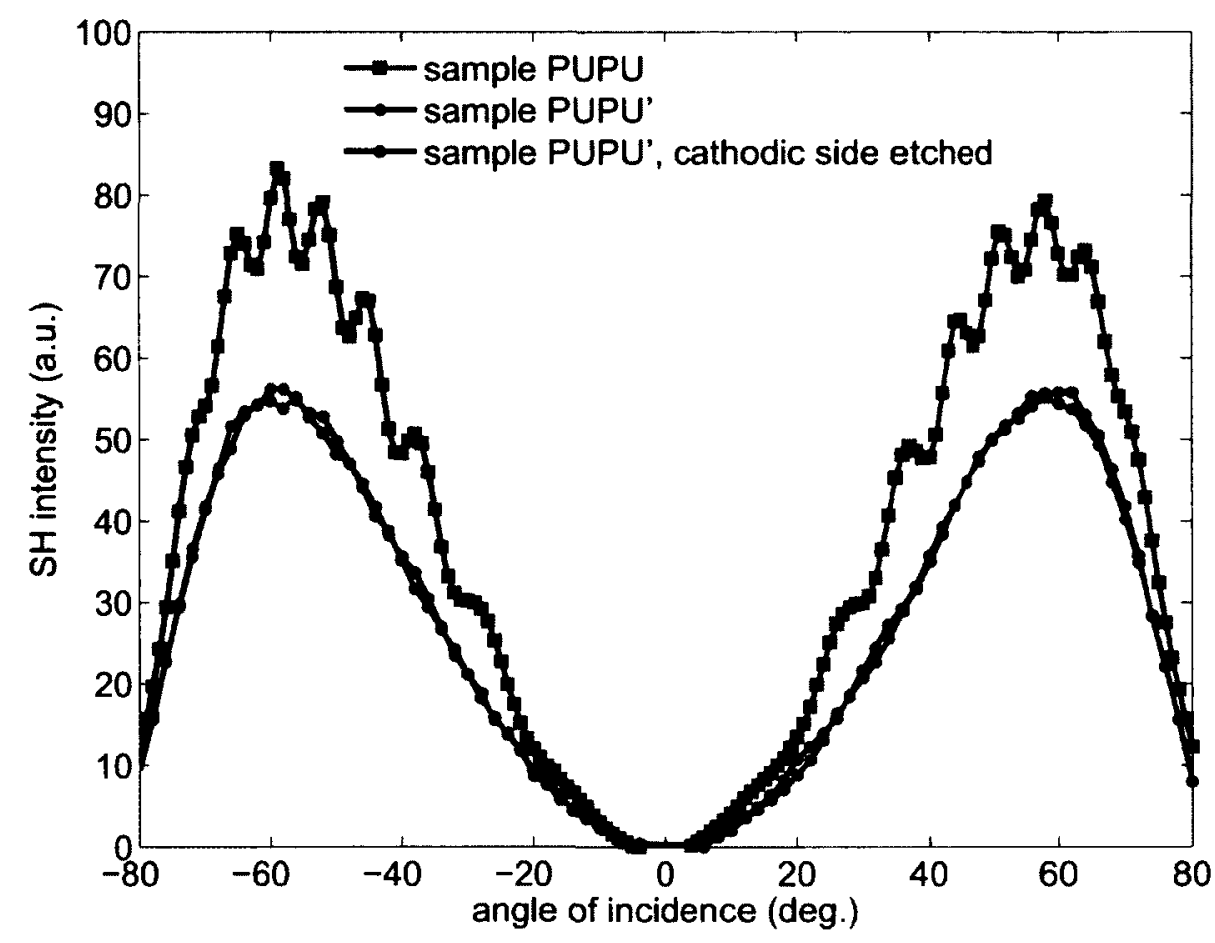

Figure 5.7: Experimentally measured SHG in corona poled multilayered samples PUPU and PUPU' (PUPU with backside layers removed).

peaks. Second, consider the cathodic side - in bulk glass, the impurity ions move away from the anode and accumulate in a very thin layer at the cathode [72] [150], where they are possibly reduced to form atoms [151]. In essence, once they move away from the anodic region, they no longer contribute to the magnitude of the induced nonlinearity. In contrast, the existence of charge-trapping layers ensures that these ions remain within and contribute to the formation of the nonlinear region. Therefore, similarly to the anodic side, the phosphorus-doped layers at the cathodic side cause the positive alkali ions to accumulate in their vicinity, recreating a capacitor-like structure that results in the simultaneous poling of the cathodic layered region.

\subsection{Summary and Implications}

The results of this chapter indicate that silica-based multilayered structures can be used as an effective tool to enhance the nonlinearity in corona poled glass devices. 
The most important findings of this chapter are:

1. Thin-film doped silica multilayered design can be used to enhance the effective second-order nonlinearity induced by corona poling by an order of magnitude compared to poling of bulk silica glass. In particular, trapping of positive charges by phosphorus-doped silica layers is believed to be responsible for the improvement in the induced nonlinearity.

2. When the poled structure consists of multiple multilayered sections, the poling voltage was shown to be divided between them, realizing simultaneous poling of multiple glass regions.

The ability to pole multiple regions of glass simultaneously is unique to the multilayered thin-film structures, and is an important step towards enhancing the effective poling-induced nonlinearity by increasing the total thickness over which the nonlinearity occurs. Moreover, the 14-fold difference in the SHG in identical structures with the exception of the dopant used (phosphorus vs. boron) clearly indicates that design optimizations are possible. Extending the ideas presented in this chapter to larger multilayered stacks with optimized dimensions and doping levels is expected to result in even stronger enhancement, paving the way for a practical electro-optic modulator in silica glass that is compatible with standard planar lightwave circuit technology. 


\section{Chapter 6}

\section{Investigation of Thermally Poled Multilayered Structures}

Thermal poling is the most commonly used poling method for inducing secondorder nonlinear effects in glasses. As previously described in Chapter 3, this phenomenon is believed to be a consequence of electric field-induced ion migration within the glass network. The main goal of this chapter is to find out whether doped silica multilayered structures can be used to control the migration of charges during poling such that a strong nonlinear region is created over a controllable thickness.

This chapter begins with the description of baseline measurements of the SHG in bulk glass that has been thermally poled (section 6.1) and in unpoled multilayered structures (section 6.2). Section 6.3 describes the first part of the thermal poling studies, which focuses on the effect of dopants on the induced nonlinearity in multilayered structures with a few thick (micrometer-scale) layers. The second part of the thermal poling studies, presented in section 6.4, explores the induced nonlinearity in structures with a large number of thin (sub-100 nm-scale) layers. Finally, section 6.5 summarizes the results presented in this chapter, and discusses their implications on the overall design of efficient second-order nonlinear glass structures. 


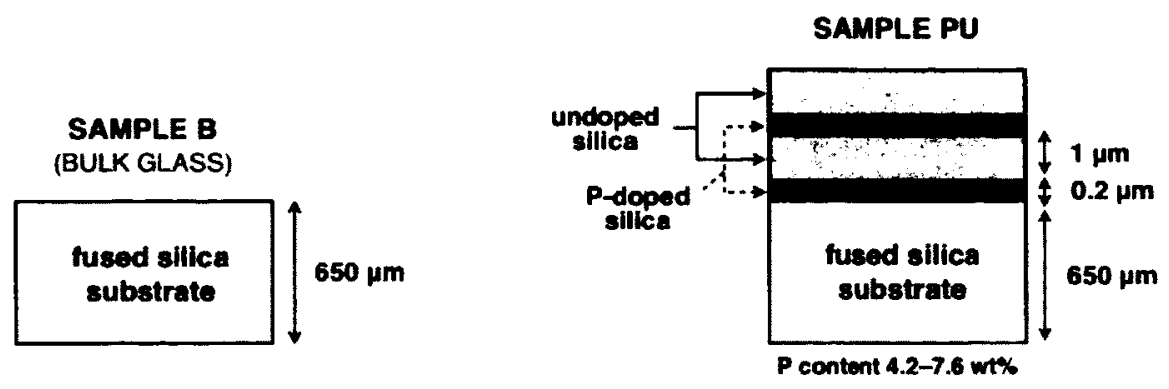

(a)

(b)

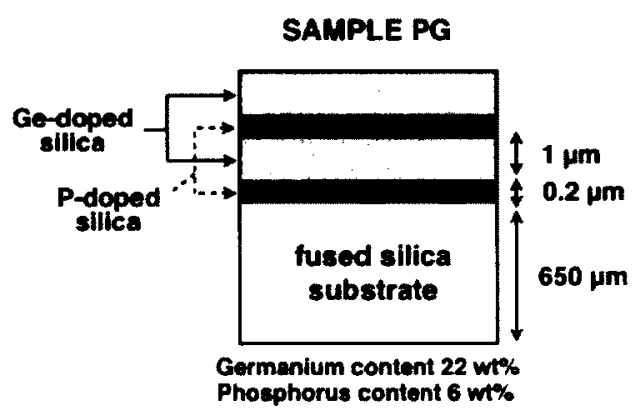

(c)

Figure 6.1: Thermally poled structures with micrometer-scale layers: (a) a bulk glass sample, (b) a four-layered stack of alternating undoped and phosphorus-doped layers, (c) a four-layered stack of alternating germanium-doped and phosphorus-doped layers.

\subsection{Thermally Poled Bulk Glass}

As was discussed in chapter 4, the nonlinear properties of the samples considered in this thesis are characterized by comparative measurements of the SHG in the multilayered structures and in bulk glass. To obtain a reference measurement, the first thermal poling experiment involved poling of a bulk fused silica sample (a pristine Suprasil 300 substrate; see sample B in Figure 6.1). The poling conditions were previously described in section 4.2 , and remained constant for all the samples studied in this chapter. Figure 6.2 shows the SHG in sample B as a function of the angle between the pump beam and the sample normal. Since Maker fringe measurements do not yield a unique profile of the induced nonlinearity [101], localized regions of the poled sample were etched using the setup described in section 4.3 to study the 
$\chi^{(2)}$ distribution. The etching experiments indicate that the nonlinearity in sample B spans over $\sim 46 \mu \mathrm{m}$, consistent with previous reports on Suprasil glass (e.g. [134]) that reported a long nonlinearity tail that spanned tens of micrometers. In a standard Maker fringe experiment (e.g. with a nanosecond laser source) this nonlinearity profile would have resulted in significant modulation fringes. However, in our case an ultrafast laser is used, and therefore the fringes are invisible due to the reduced interaction between the free and the bound second harmonic waves [144].

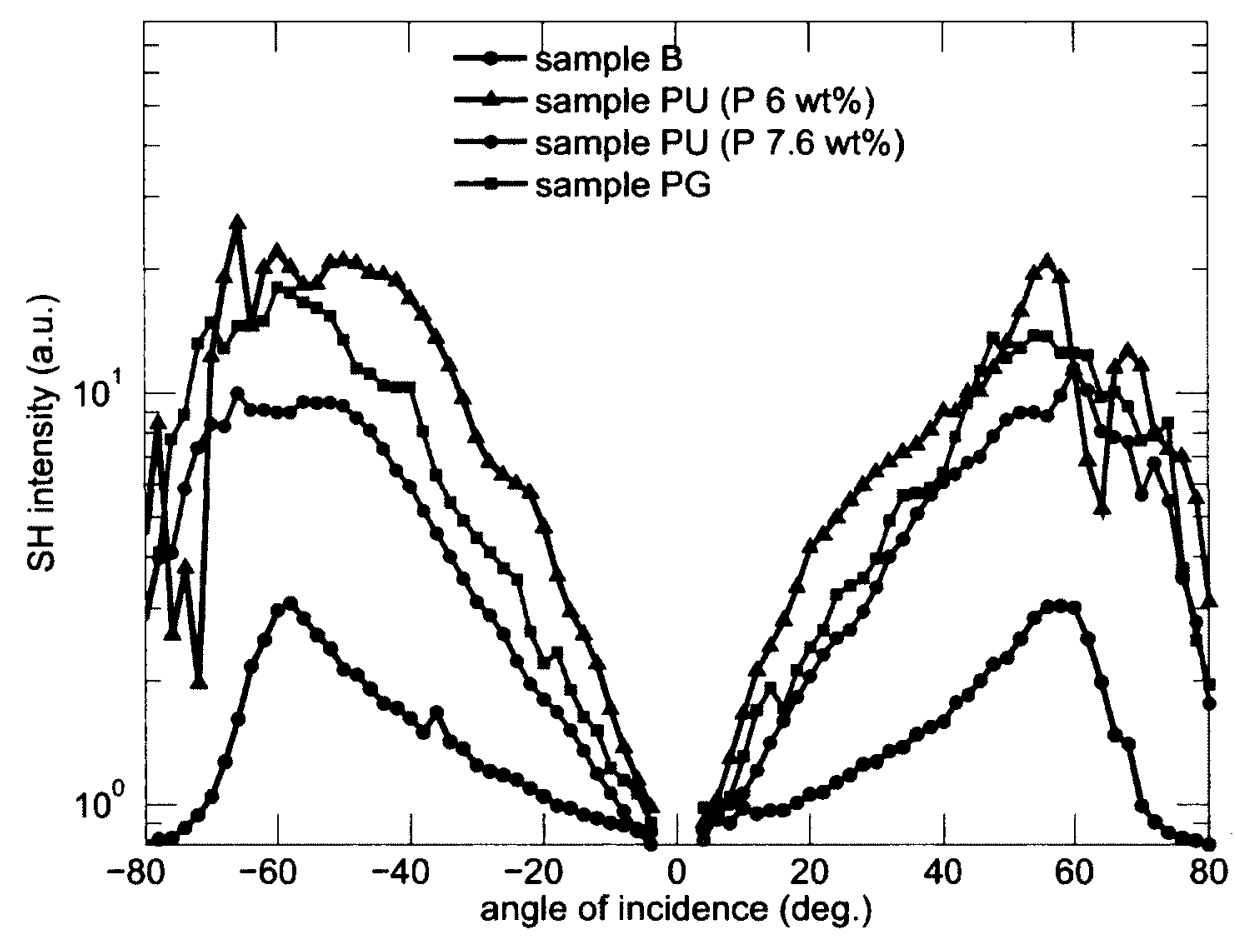

Figure 6.2: Experimentally measured SHG in thermally poled multilayered structures with micrometer-scale layers shown in Figure 6.1.

Before proceeding with the results of the multilayered glass samples, an important point must be addressed regarding the comparative SHG measurements that are used for the nonlinear characterization. The oscillating behavior of the Maker fringe measurement is due to the $\left[\frac{\sin (\Delta k L / 2)}{\Delta k L / 2}\right]^{2}$ term in equation (2.34). Using equation 2.14 
and setting the nonlinear pathlength to $L / \cos \vartheta$, this term can be expressed as:

$$
\left[\frac{\sin \left(\frac{\Delta k L}{2}\right)}{\frac{\Delta k L}{2}}\right]^{2}=\operatorname{sinc}^{2}\left(\frac{\Delta k L}{2}\right)=\operatorname{sinc}^{2}\left(\frac{\pi}{2} \frac{L / \cos \vartheta}{L_{c}}\right)
$$

where $L_{c}$ is the coherence length in fused silica, which was calculated and verified experimentally in section 5.1 to be $\sim 12 \mu \mathrm{m}$. When the pathlength is small compared to the coherence length (i.e. $L / \cos \vartheta \ll L_{c}$ ), this term approaches 1 and the second harmonic intensity scales with $\left(\chi_{\mathrm{eff}} L / \cos \vartheta\right)^{2}$. To illustrate this case, Figure 6.3(a) shows simulated waveforms of the Maker fringe SHG in nonlinear media that are thinner than $L_{c}$. As will be shown later in this chapter, the poled multilayered structures fall into this category, and thus a larger peak in the SHG measurements corresponds to a higher effective nonlinearity. However, in the case of sample $B$, the etching experiments show that the thickness of the induced nonlinearity is larger than the coherence length. When this is the case, the peak of the Maker fringe SHG measurement may not be an accurate representation of the induced nonlinearity. This occurs because widely spaced fringes that are located at different angular positions are affected unevenly by the angle-dependent terms in equation $(2.34)\left(B(\theta), \chi_{\text {eff }}^{2}(\theta)\right.$, $T_{\omega}^{2}(\theta)$ and $\left.T_{2 \omega}(\theta)\right)$. Figure 6.3(b) illustrates this scenario by looking at a few cases of simulated Maker fringe SHG waveforms for nonlinear regions that are thicker than the coherence length. Note that the simulations use the theoretical quasi-cw description presented in section 2.2.3, and therefore predict fringes that are not visible in our ultrafast SHG measurements (for a more detailed discussion on this topic see section 4.3). Nonetheless, even though the experimental measurements lack the fringes, the above argument regarding the peak of the Maker fringe envelope still applies. Therefore, for cases when the nonlinearity extends beyond a single coherence length, the peak of the Maker fringe envelope may be low not only as a result of a weak $\chi^{(2)}$, but also as a result of the angular location of the (damped) fringes. In the specific case of sample B, the nonlinearity extends over $46 \mu \mathrm{m}$, which according to Figure 6.3(b) is close to the $50 \mu \mathrm{m}$ thickness that results in the highest peak (with the intensity difference between the two cases being at most $7 \%$, within the experimental error of these measurements). Therefore the second harmonic signal generated in sample $B$, 
as shown in Figure 6.2, truthfully represents the nonlinearity induced in the sample (as opposed to a "coincidentally" low value due to the angular location of its fringes) and can be compared with the SHG measurements obtained in subsequent sections.

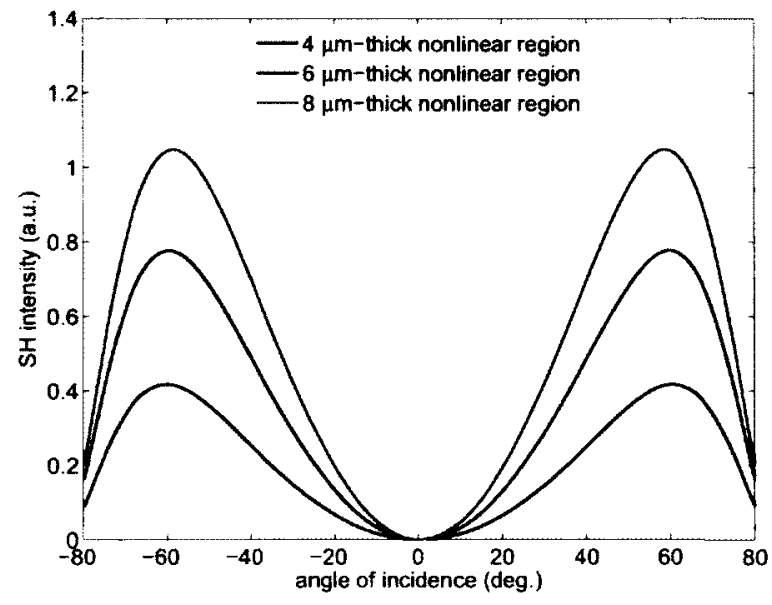

(a)

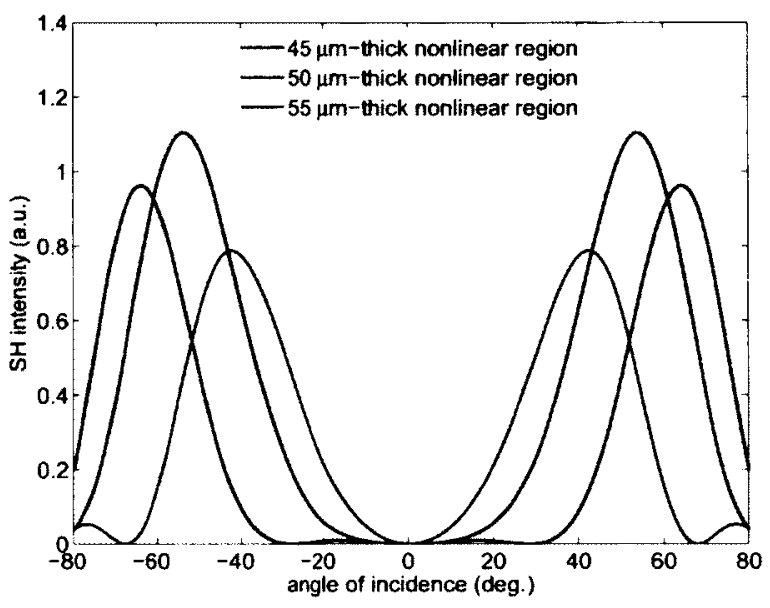

(b)

Figure 6.3: Simulated SHG in structures with varying thicknesses of the nonlinear region: (a) nonlinear interaction region smaller than the coherence length, (b) nonlinear interaction region larger than the coherence length.

Note that unlike the results presented in Chapter 5, the experimental results in this chapters are mostly plotted on a logarithmic scale since the SHG in the various samples varies by more than two orders of magnitude. Moreover, although the intensities of the second harmonic signals are expressed in arbitrary units, the relative intensities are meaningful among the figures in this chapter (with the exception of the figures in section 6.3.3). The measured signals in Chapter 5, however, cannot be compared directly to the figures in this chapter since they were measured with different photomultiplier gain setting. The SHG in thermally poled bulk glass was about an order of magnitude higher than the SHG in corona poled bulk glass.

\subsection{Unpoled Multilayered Samples}

Although the main focus of this investigation is on poled glass structures, it must be noted that the introduction of interfaces breaks the symmetry of the glass struc- 
ture, and thus a non-zero second harmonic signal can be expected in the multilayered samples even without poling [152]. In order to gauge the magnitude of this effect, the SHG in all of the multilayered structures was measured prior to poling. The second harmonic signal in these unpoled samples was hardly detectable with our measurement setup, and was found to be more than two orders of magnitude lower than the SHG in the poled sample $B$.

\subsection{Thermally Poled Multilayered Structures with Thick Layers}

\subsubsection{Effect of Dopants}

In the first part of our work, structures with a few thick layers were investigated to study the effect of dopant composition. Parts (b) and (c)' of Figure 6.1 show the two main designs of the multilayered structures, along with dopant concentrations as obtained by energy-dispersive x-ray spectroscopy (EDX). The measured SHG in these structures is shown in Figure 6.2. Compared to bulk glass poled under identical conditions, the SHG in sample PU (with 6 wt\% phosphorus concentration) is higher by a factor of 8.4, while the SHG in sample PG is higher by a factor of 5.9.

The SHG in sample PU, consisting of alternating layers of undoped and phosphorusdoped (6 wt\%) layers, is the strongest out of the four samples shown in Figure 6.2. However, it is not clear why this sample exhibits irregular fluctuations in the Maker fringe envelope. To investigate whether they are caused by scattering in the deposited glass, two linear transmission experiments were preformed. The first experiment involved measuring the linear transmission at $800 \mathrm{~nm}$ (the fundamental source wavelength). The setup used for the experiment and the corresponding transmission characteristics are shown in Figure 6.4. The second experiment, presented in Figure 6.5, involved measuring the linear transmission at $400 \mathrm{~nm}$ (the second harmonic wavelength).

Both the linear transmission measurements show the expected relationship be- 


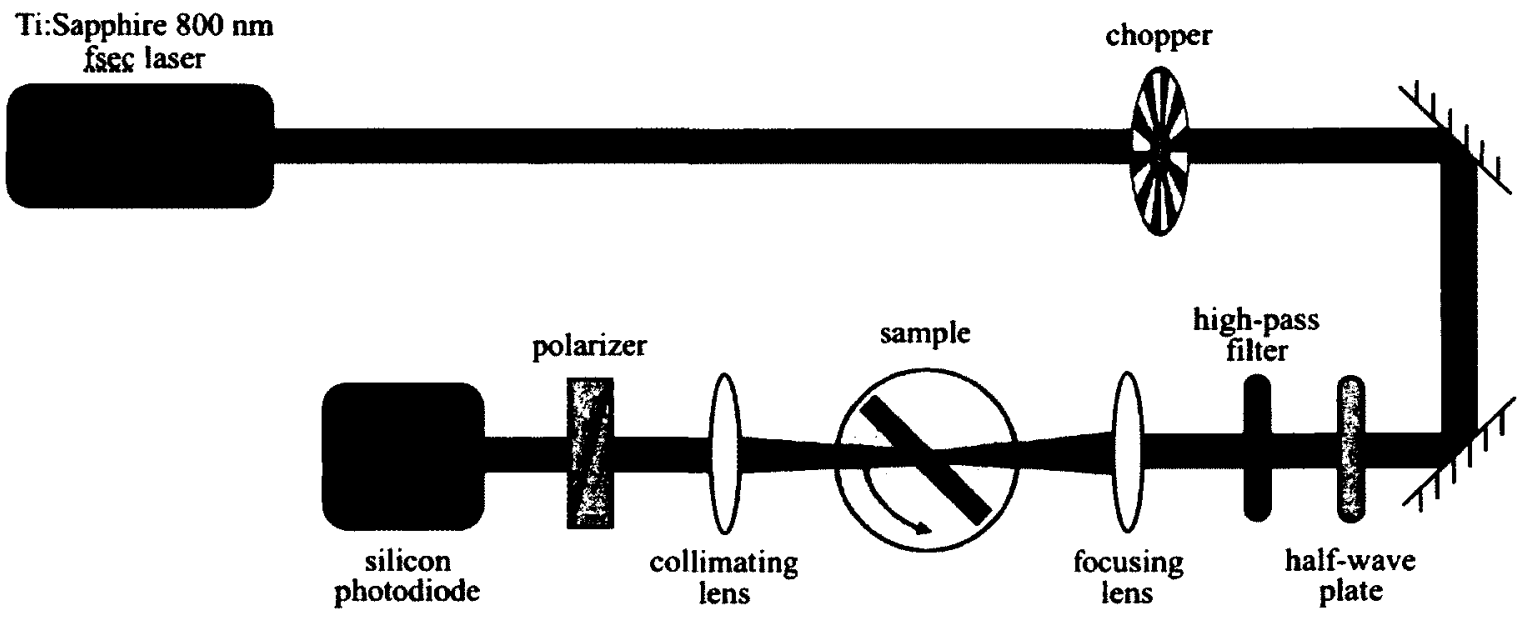

(a)

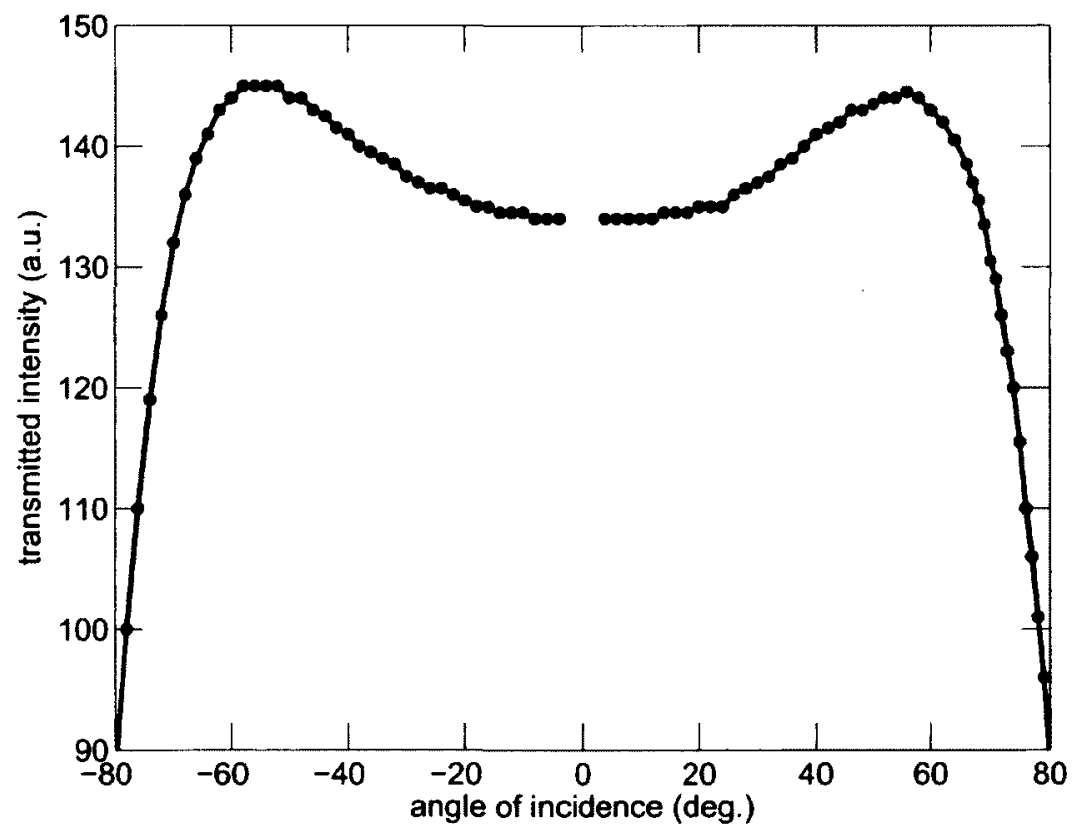

(b)

Figure 6.4: Linear transmission characterization of sample PU at $800 \mathrm{~nm}$ : (a) the experimental setup, (b) the linear transmission as a function of incidence angle.

tween the intensity and the angle of incidence that results from the angular dependence of the Fresnel transmission factor $T_{\omega}^{2}(\theta) T_{2 \omega}(\theta)$ described in section 2.2.3. The smooth shape of the two curves indicates that there is no significant scattering. Therefore, the fluctuations in the SHG observed in Figure 6.2 are due to local variations in 
the poling-induced nonlinearity. Such variations may be the result of defects that are incorporated into the glass layers during the PECVD process [61] [153]. When such defects are distributed non-uniformly, they can contribute to sudden fluctuations in the generated second harmonic signal.

The enhancement in the poling-induced nonlinearity shown in Figure 6.2 may be attributed to the presence of phosphorus-doped films. When phosphorus incorporates into the silica glass matrix, it primarily oxidizes in pentavalent form $\left(\mathrm{P}_{2} \mathrm{O}_{5}\right)$ and substitutes for $\mathrm{Si}^{4+}$ in the $\mathrm{SiO}_{4}$ tetrahedra. Because phosphorus has an extra valence electron compared to silicon, non-bridging oxygen ions become associated with phosphorus sites [149]. These oxygen ions have local negative charges, and can trap any positive charges moving through the lattice. Thus the detrimental positive charges that are injected from the anode are trapped by the non-bridging oxygen ions, resulting in the nonlinearity enhancement that is seen in our experiments.

\subsubsection{Effect of Annealing}

Thin films deposited by chemical vapor deposition methods are usually annealed to reorder the glass structure, reduce the film defects, relieve film stresses, and stabilize the refractive index. Although in this work most of the samples were poled as-is after deposition, a number of samples were annealed prior to poling to study the effect of annealing on the induced nonlinearity. The annealing step involved a 4 hour bake in nitrogen at $950{ }^{\circ} \mathrm{C}$. As shown in Figure 6.6 the SHG in the annealed samples $\mathrm{PU}$ and PG reduced by factors of 5.2 and 2.9 , respectively, compared to unannealed samples. The much smoother Maker fringe envelopes of the annealed samples confirms the hypothesis that the irregular fluctuations in the unannealed samples are due to structural defects, which are reduced during the annealing process.

The PECVD system used for the deposition of these multilayered samples is optimized for very high deposition rates, and therefore may result in glass inhomogeneity that is the likely cause of the irregular fluctuations in unannealed samples. Further study is required to determine whether these fluctuations are detrimental for the design of practical poled glass devices. In the context of electro-optic phase shifting, 


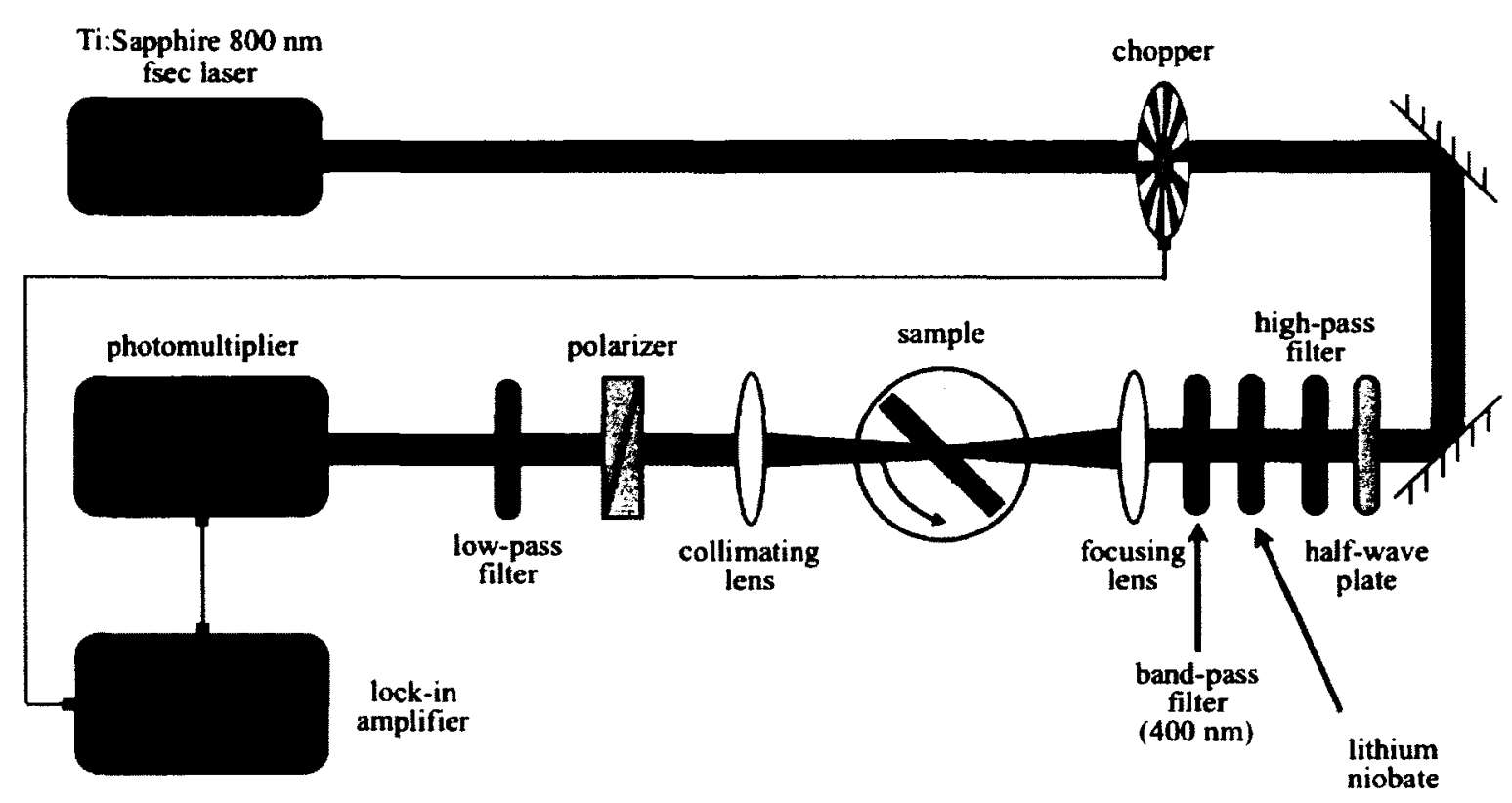

(a)

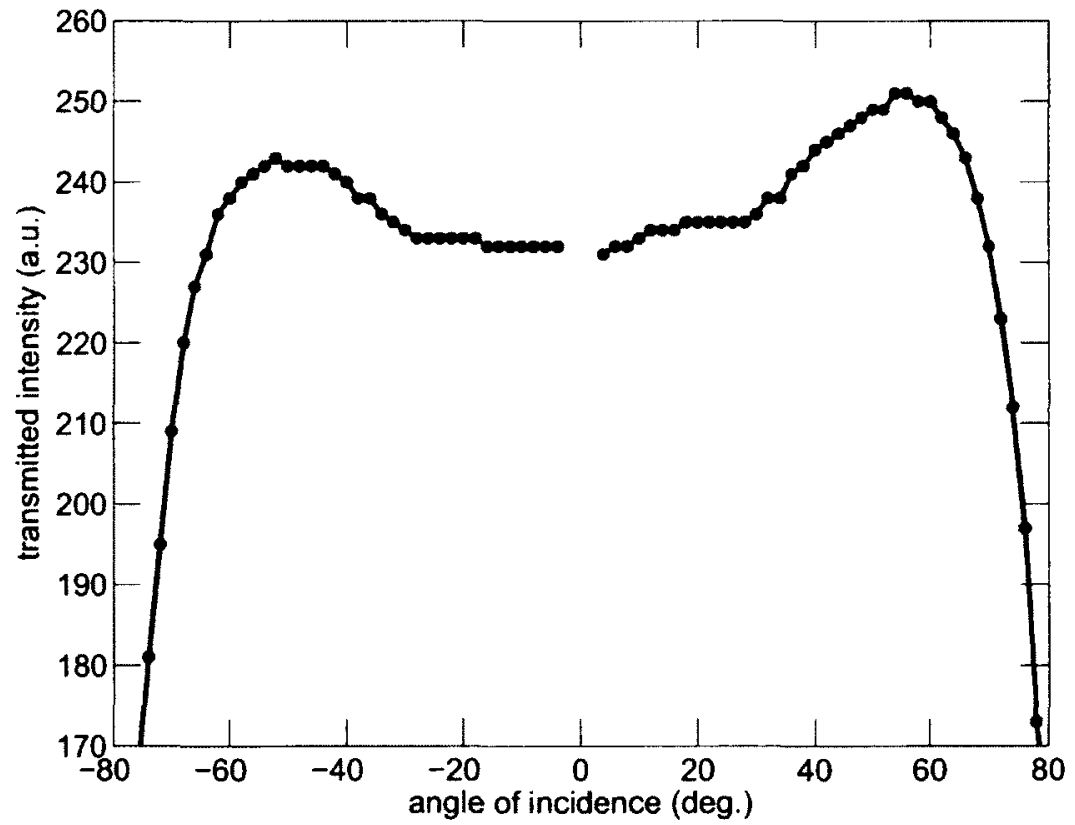

(b)

Figure 6.5: Linear transmission characterization of sample PU at $400 \mathrm{~nm}$ : (a) the experimental setup, (b) the linear transmission as a function of incidence angle. 
it is the "average" second-order nonlinearity that determines the overall phase shift, therefore the fluctuations may not be a major concern. However, for SHG applications where quasi-phase matching is required, these irregularities will impair the phase matching. The propagation loss properties of such unannealed films is also an important parameter that needs to be characterized. That being said, there do exist PECVD processes, which can be used to realize planar waveguides with very low propagation losses, that do not require annealing [154]. Such deposition processes may prove to be more optimal for the fabrication of multilayered structures with high poling-induced nonlinearities.

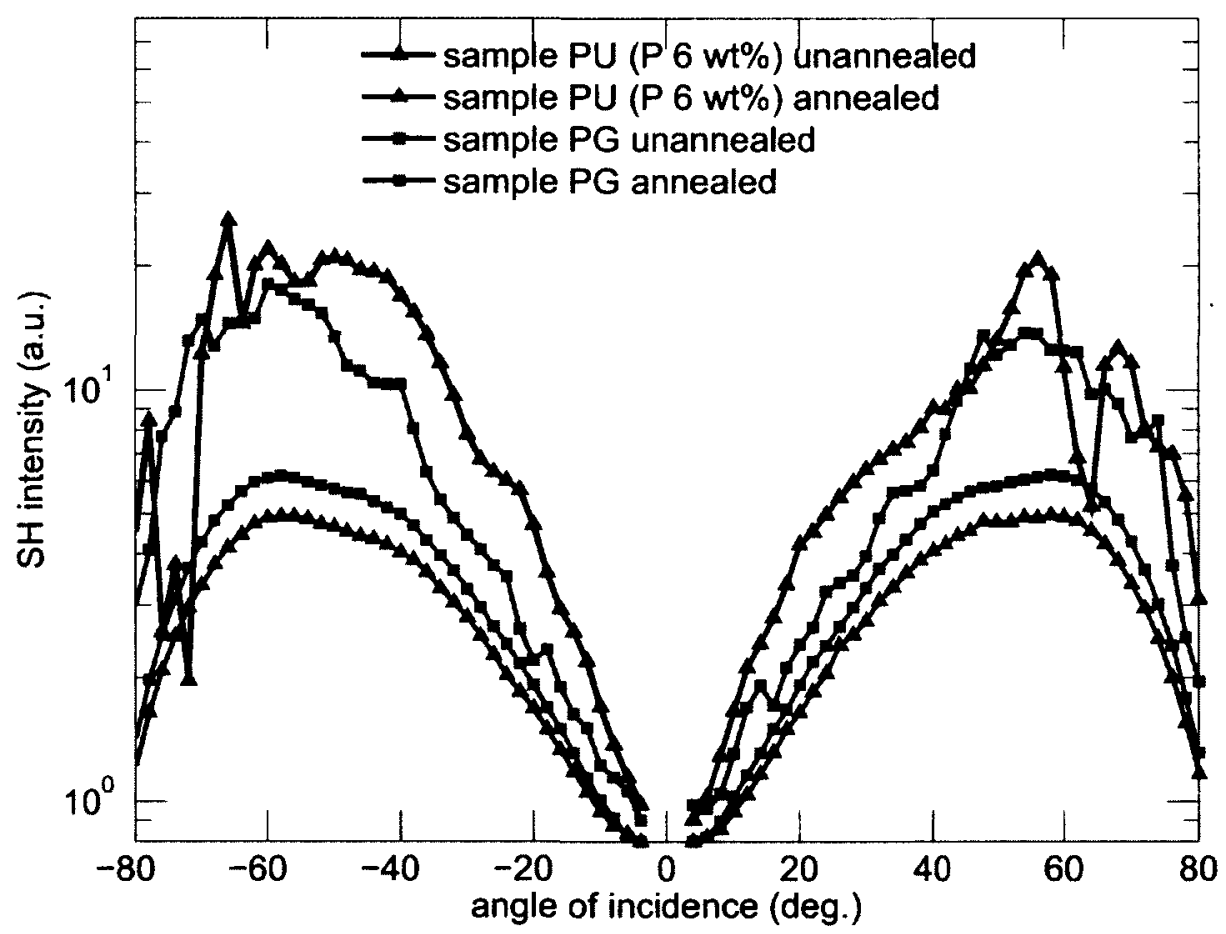

Figure 6.6: Comparison of the experimentally measured SHG in as-deposited and annealed multilayered structures.

\subsubsection{Evidence of Non-Uniform Frozen-In Electric Field Tilt}

The experimental measurements performed as part of this investigation show that the SHG in poled bulk glass varies very little throughout the poled region (except 


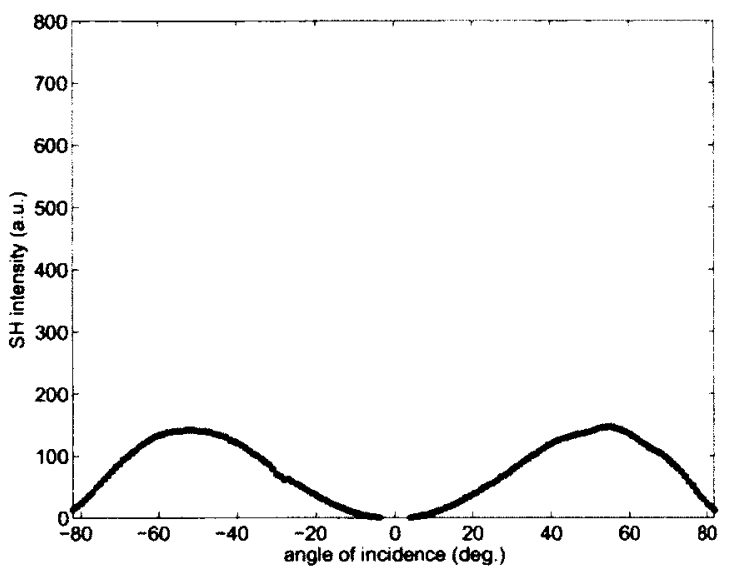

Figure 6.7: Spatially-invariant, symmetric Maker fringe envelope characteristic of thermally poled bulk glass samples.

when measuring close to the electrode edges). Recall from Chapter 3 that the cause of the induced nonlinearity is the migration of impurity ions during the poling process. The electric field that forms as a result of the charge distribution within the glass interacts with the third-order nonlinear susceptibility to result in an effective $\chi^{(2)}$. The orientation of this frozen-in electric field is perpendicular to the sample surface, and the reduced second-order susceptibility tensor that can be used to describe the nonlinear structure of poled glass is given by:

$$
\left[\begin{array}{cccccc}
0 & 0 & 0 & 0 & \chi_{31} & 0 \\
0 & 0 & 0 & \chi_{31} & 0 & 0 \\
\chi_{31} & \chi_{31} & \chi_{33} & 0 & 0 & 0
\end{array}\right]
$$

The theoretical expression for the second harmonic intensity includes a term that represents the effective second-order susceptibility coefficient $\chi_{\text {eff }}$ (see equation (2.12)). The form of $\chi_{\text {eff }}$ depends on the principal axes of the crystalline structure, the rotation axis of the Maker fringe measurement, and the polarization of the fundamental beam. It was previously shown that the effective second-order susceptibility coefficients for poled glass are:

$$
\begin{aligned}
& \chi_{e f f, p}=3 \chi_{31} \sin \vartheta \cos ^{2} \vartheta+\chi_{33} \sin ^{3} \vartheta \\
& \chi_{e f f, s}=\chi_{31} \sin \vartheta
\end{aligned}
$$


for the two polarizations of the fundamental beam, where $\vartheta$ is the internal propagation angle of the beam. Due to the form of the effective nonlinear coefficients, when the second harmonic signal generated in the glass sample is measured as a function of the beam incidence angle, the signal is expected to be symmetric about normal incidence. Indeed, when a bulk glass sample is thermally poled and characterized, a symmetric envelope typical of the one shown in Figure 6.7 is observed.

However, unlike the case of bulk glass, the second harmonic signal generated in thermally poled multilayered samples shows an interesting dependence on the measurement location within the poled region. The results presented here pertain to a multilayered structure PU (see Figure 6.1(b)) with $4.2 \mathrm{wt} \%$ phosphorus doping concentration. Four examples of the spatially-varying results observed in this sample are shown in Figure 6.8(b)-(e), with the corresponding locations indicated in Figure 6.8(a).

The experimental evidence presented in Figure 6.8 suggests that, when measured away from the centre of the poled region, the frozen-in field in the thermally poled multilayered samples is not perpendicular to the sample surface. The merit of this idea was tested by developing a model based on the assumption that the second-order nonlinear structure of the resultant material can be described by the reduced tensor given in equation (6.2) rotated by an angle $\varphi$ (where $\varphi$ is the position-dependent angular tilt of the frozen-in field). The rotated elements of the $\chi^{(2)}$ tensor (in nonreduced form) can be obtained by using the transformation law [18] [155]:

$$
\chi_{\mu \alpha \beta}^{(2)^{\prime}}=R_{\mu u} R_{\alpha a} R_{\beta b} \chi_{u a b}^{(2)}
$$

where summation over repeated indices is assumed. The $R_{i j}$ are the elements of a three-dimensional rotation matrix, which is given by:

$$
R_{x}=\left[\begin{array}{ccc}
1 & 0 & 0 \\
0 & \cos \varphi & -\sin \varphi \\
0 & \sin \varphi & \cos \varphi
\end{array}\right] \quad R_{y}=\left[\begin{array}{ccc}
\cos \varphi & 0 & \sin \varphi \\
0 & 1 & 0 \\
-\sin \varphi & 0 & \cos \varphi
\end{array}\right]
$$

for rotation about the $x$ and $y$ axes, respectively. For example, the $\chi_{113}^{(2)}$ element in 


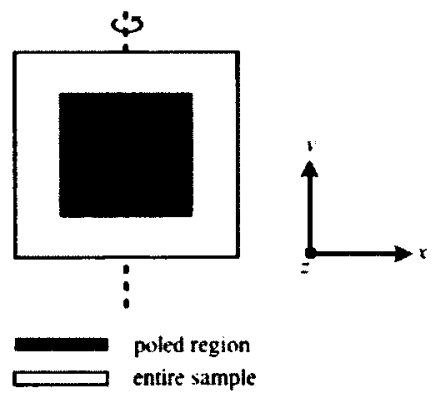

(a)

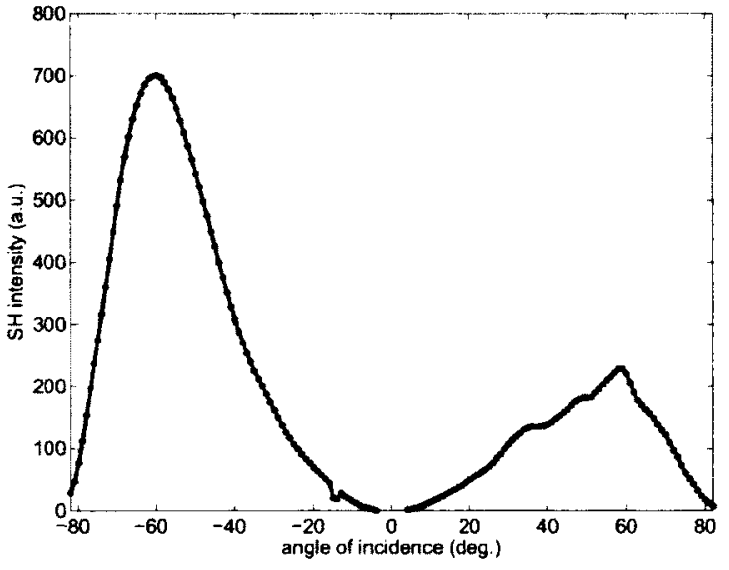

(b)

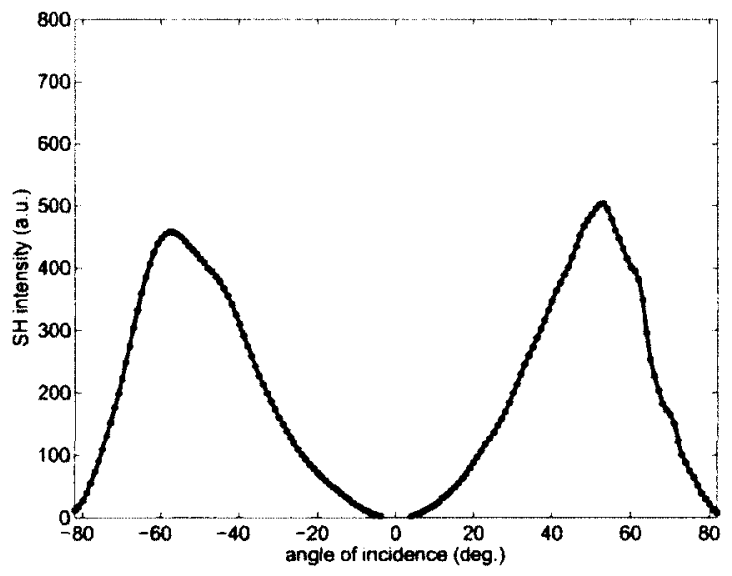

(d)

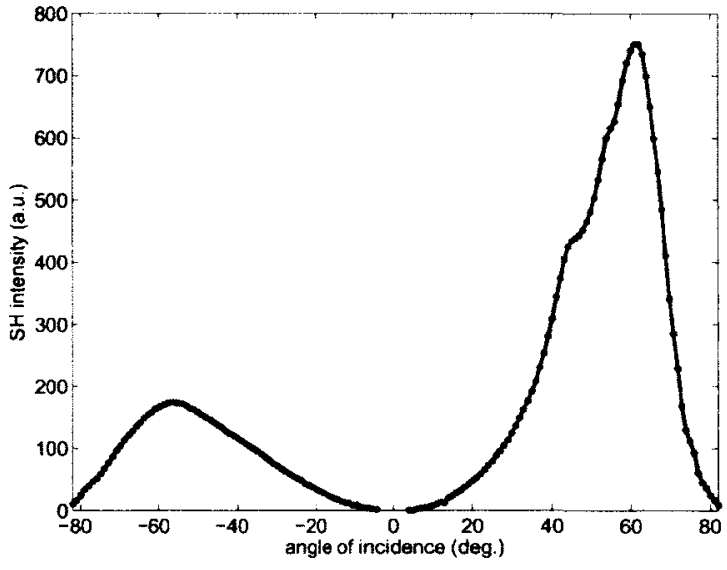

(c)

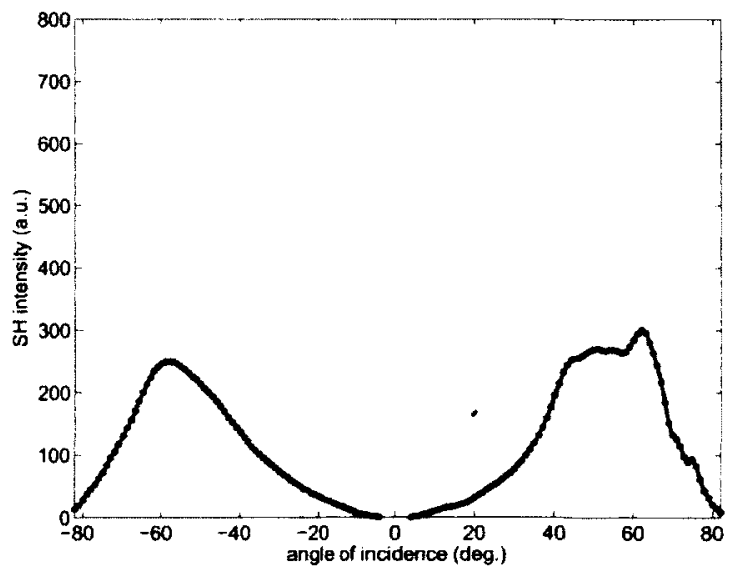

(e)

Figure 6.8: Spatially-varying Maker fringe envelope characteristic of thermally poled multilayered glass samples. (a) Locations of the Maker fringe measurements corresponding to the results presented in parts (b)-(e). (b) Measurement taken at location I. (c) Measurement taken at location II. (d) Measurement taken at location III. (e) Measurement taken at location IV. 
the rotated coordinate system takes the form:

$$
\chi_{113}^{(2)^{\prime}}=\sum_{u=1}^{3} \sum_{a=1}^{3} \sum_{b=1}^{3} R_{1 u} R_{1 a} R_{3 b} \chi_{u a b}^{(2)}
$$

A similar operation must be repeated for each of the 27 components of the third rank tensor $\chi_{i j k}^{(2)}$. This was computed symbolically using Mathematica. Assuming that the frozen-in electric field is tilted by $\varphi$ about the $y$-axis (where the coordinate system is shown in Figure 6.8(a)), the rotated reduced tensor takes the form:

$$
\begin{aligned}
& \chi^{(2)^{\prime}}=\left[\begin{array}{llllll}
\chi_{11}^{\prime} & \chi_{12}^{\prime} & \chi_{13}^{\prime} & \chi_{14}^{\prime} & \chi_{15}^{\prime} & \chi_{16}^{\prime} \\
\chi_{21}^{\prime} & \chi_{22}^{\prime} & \chi_{23}^{\prime} & \chi_{24}^{\prime} & \chi_{25}^{\prime} & \chi_{26}^{\prime} \\
\chi_{31}^{\prime} & \chi_{32}^{\prime} & \chi_{33}^{\prime} & \chi_{34}^{\prime} & \chi_{35}^{\prime} & \chi_{36}^{\prime}
\end{array}\right] \\
& \chi_{11}^{\prime}=3 \chi_{31} \cos ^{2} \varphi \sin \varphi+\chi_{33} \sin ^{3} \varphi \\
& \chi_{12}^{\prime}=\chi_{31} \sin \varphi \\
& \chi_{13}^{\prime}=\sin \varphi\left[\left(-2 \chi_{31}+\chi_{33}\right) \cos ^{2} \varphi+\chi_{31} \sin ^{2} \varphi\right] \\
& \chi_{14}^{\prime}=\chi_{16}^{\prime}=0 \\
& \chi_{15}^{\prime}=\cos \varphi\left[\chi_{31} \cos ^{2} \varphi+\left(-2 \chi_{31}+\chi_{33}\right) \sin ^{2} \varphi\right] \\
& \chi_{21}^{\prime}=\chi_{22}^{\prime}=\chi_{23}^{\prime}=\chi_{25}^{\prime}=0 \\
& \chi_{24}^{\prime}=\chi_{31} \cos \varphi \\
& \chi_{26}^{\prime}=\chi_{31} \sin \varphi \\
& \chi_{31}^{\prime}=\cos \varphi\left[\chi_{31} \cos ^{2} \varphi+\left(-2 \chi_{31}+\chi_{33}\right) \sin ^{2} \varphi\right] \\
& \chi_{32}^{\prime}=\chi_{31} \cos \varphi \\
& \chi_{33}^{\prime}=\chi_{33} \cos ^{3} \varphi+3 \chi_{31} \cos \varphi \sin ^{2} \varphi \\
& \chi_{34}^{\prime}=\chi_{36}^{\prime}=0 \\
& \chi_{35}^{\prime}=\sin \varphi\left[\left(-2 \chi_{31}+\chi_{33}\right) \cos ^{2} \varphi+\chi_{31} \sin ^{2} \varphi\right]
\end{aligned}
$$

As expected, the above reduces to the usual (i.e. non-rotated) form of the reduced $\chi^{(2)}$ tensor given by equation (6.2) when $\varphi=0$.

Now that the reduced susceptibility tensor is known, the derivation presented in section 2.2 .3 can be used to obtain the values of the effective susceptibility coefficients. 
Consider a $p$-polarized fundamental beam that is incident on the sample at an external angle $\theta$ that results in an internal angle of propagation $\vartheta$, a Maker fringe measurement where the sample is rotated about a vertical axis as shown in Figure 6.8(a), and a frozen-in electric field that is tilted by $\varphi$ relative to the sample normal. In this case, the effective susceptibility coefficient takes the form:

$$
\chi_{e f f, p}^{\prime}=\frac{1}{2}\left\{2 \chi_{31}+\chi_{31}+\left(3 \chi_{31}-\chi_{31}\right) \cos [2(\vartheta+\varphi)]\right\} \sin (\vartheta+\varphi)
$$

To gain a more intuitive understanding of what the above expression means, note that the effective nonlinear coefficients are related by $\chi_{33}=3 \chi_{31}$ when conditions for Kleinman symmetry hold [156] [18] (a relationship that was confirmed in multiple poled glass experiments, e.g. [64]). In this case equation (6.9) simplifies to $\chi_{e f f, p}^{\prime}=3 \chi_{31} \sin (\vartheta+\varphi)$ (compared to $\chi_{e f f, p}^{\prime}=3 \chi_{31} \sin (\vartheta)$ when the frozen-in field is perpendicular to the sample surface). In other words, rotation of the $\chi^{(2)}$ tensor is equivalent to shifting the zero-point of the Maker fringe measurement. The same intuitive physical description applies to the case of an $s$-polarized fundamental beam, where the effective susceptibility coefficient is derived to be:

$$
\chi_{e f f, s}^{\prime}=\chi_{31} \sin (\vartheta+\varphi)
$$

Therefore, for a $p$-polarized fundamental beam, a frozen-in field oriented at an angle $\varphi$ relative to the sample normal implies that the measurements of the second harmonic signal at the locations indicated in Figure 6.8(a) have the following properties:

LOCATIONS I AND II: to the right and the left off the centre of the poled region, the zero-point of the Maker fringe measurement is shifted by $\varphi$, resulting in an asymmetry between the positive and the negative measurement angles.

LOCATION III: in the centre of the poled region, the frozen-in field is perpendicular to the surface, and the Maker fringe waveform resembles the standard case of poled bulk glass.

LOCATION IV: above or below the centre, the angular orientation of the frozenin field does not change as the sample is rotated, and thus no asymmetry is observed. 
Note that there is a qualitative agreement between the predictions of the mathematical model and the experimental measurements presented in Figure 6.8. However, quantitative agreement at the Maker fringe zero-point has not been achieved. As demonstrated in Figure 6.9, the model predicts a non-zero second harmonic signal at normal incidence, while the experiments show a vanishing signal when the incidence angle is zero. This discrepancy is probably related to the initial assumption that the poled multilayered structure can be described by the reduced tensor given by equation (6.2) rotated by $\varphi$. In reality, the effect of the layers is more complex, and thus the nonlinear susceptibility tensor is likely to contain additional $\chi_{i j k}^{(2)}$ elements that were not considered in this model.

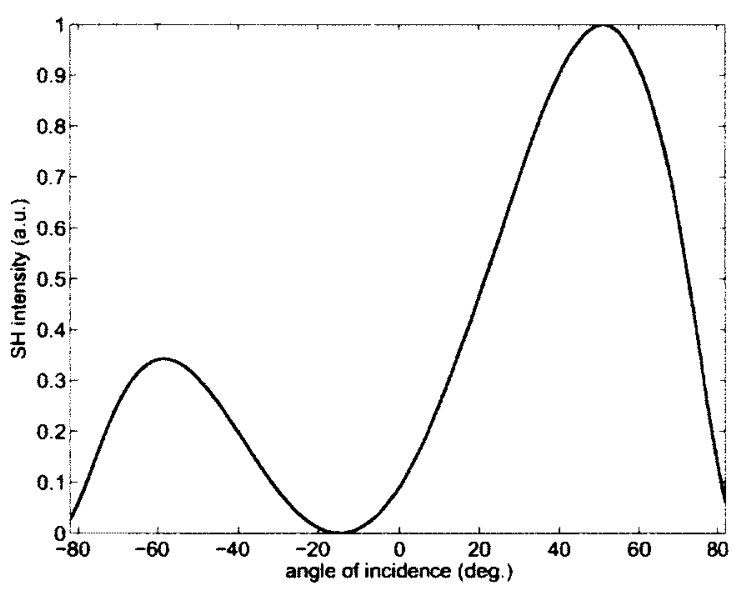

Figure 6.9: Predicted Maker fringe envelope for a nonlinear material characterized by the $\infty \mathrm{mm}$ crystal group rotated by $10^{\circ}$.

Although an accurate mathematical model was not obtained, the observation of a non-symmetric envelope (and therefore of a non-uniform frozen-in electric field tilt) in the thermally poled samples points to an interesting property of the multilayered design. In the case of bulk glass poling, the impurity ions migrate through a homogeneous material and move away from the depletion region; in essence they have no effect on the frozen-in electric field near the anodic surface. In contrast, the addition of a multilayered stack may hinder the migration of charges or trap them as was discussed in section 6.3.1. The notable distinction here is that the impurity ions remain within the important region near the anode, contributing to the magnitude and the 
orientation of the frozen-in field. There are a few factors that could contribute to this observation of a non-uniform tilt of the frozen-in electric field. First, unequal stress distribution in the multilayered stack may cause stress-driven diffusion of charges [157] along the layers, resulting in a non-uniform distribution of charges within the poled region. Second, if the deposited films are under compressive stresses (as is the case for most of the deposited structures; see Appendix A) that cause a substantial curvature of the wafer, minute air gaps at the outer edges of the top silicon electrode may create a non-uniform poling effect that results in a tilted frozen-in field. It is possible that the finite size of the top electrode further contributes to the unequal distribution of the positive impurity ions in the $x-y$ plane. The results presented in this section indicate that thin film silica layers can be used to exercise a higher degree of control over the migration of impurity ions, and thus over the poling-induced nonlinear properties of the resultant material. Further study of this unique property of poled multilayered structures could result in a better understanding on the effect of the interfaces, and how it can be used to engineer more efficient nonlinear structures.

\subsection{Thermally Poled Multilayered Structures with Thin Layers}

Based on the experimental results presented in section 6.3 , it was concluded that multilayered silica-based structures have the potential of increasing the poling-induced second-order nonlinearity. The next question that arose was whether multilayered stacks with the same overall thickness but a larger number of very thin layers would result in a more substantial increase in the induced effect? Although PECVD is generally not designed for the deposition of very thin layers because of its relatively high deposition rate, the system used for this project was capable of depositing sub-100 nmthick alternating layers of undoped and lightly germanium-doped silica. Therefore to answer the above question, we concentrated on the study of multilayered structures consisting of germanium-doped and undoped silica layers shown in Figure 6.10.

The measured SHG in the multilayered samples with germanium-doped and un- 
doped films is shown in Figure 6.11. The four-layered sample G resulted in only a modest improvement of a factor of two compared to bulk glass. However, when a sample with 40 nanolayers and $3 \mu \mathrm{m}$ total stack thickness (see sample $\mathrm{M}$ in Figure 6.10) was poled, a 204-fold improvement in the generated second harmonic signal was observed.

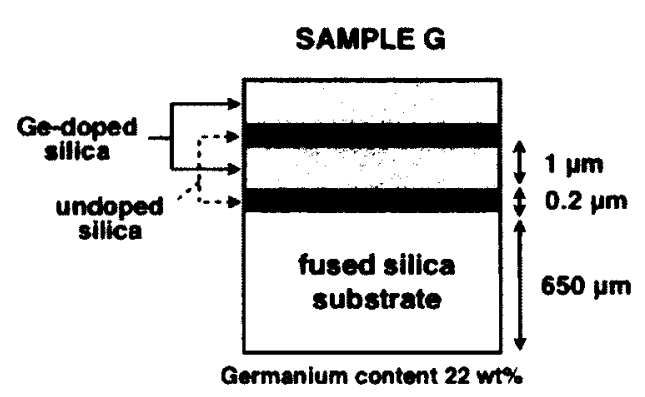

(a)

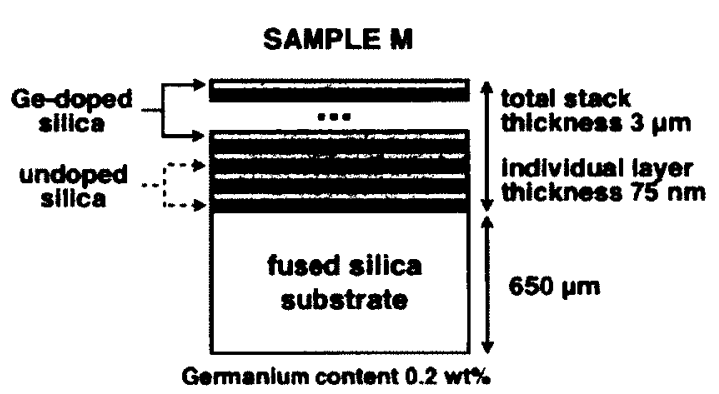

(b)

Figure 6.10: Thermally poled structures with micrometer- and sub-100 nm-scale germanium-doped layers: (a) a four-layered stack of alternating undoped and germanium-doped layers, (b) a multilayered stack consisting of a large number of alternating undoped and germanium-doped nanolayers.

To study the spatial extent of the nonlinearity in sample $M$ as a function of depth, etching experiments in dilute hydrofluoric acid on localized areas of the sample were performed using the setup described in section 4.3. After each etch, the SHG in the remaining thickness of the sample was remeasured, with the results summarized in Figure 6.12. The nonlinearity was found to be located predominantly (>95\%) in the multilayered stack, which confirms our hypothesis that silica layers can be used to achieve a high degree of control over the thickness and the location of the induced $\chi^{(2)}$.

Furthermore, the multilayered design may offer another practical advantage compared to bulk glass. The impurity levels in synthetic bulk silica, which is fabricated by the flame hydrolysis method, can vary significantly among ingots of nominally identical glass. Because the dynamics of the poling process strongly depend on the impurity content, the extent and the magnitude of the induced nonlinearity in bulk glass are difficult to control and reproduce [134]. In contrast, the PECVD process 


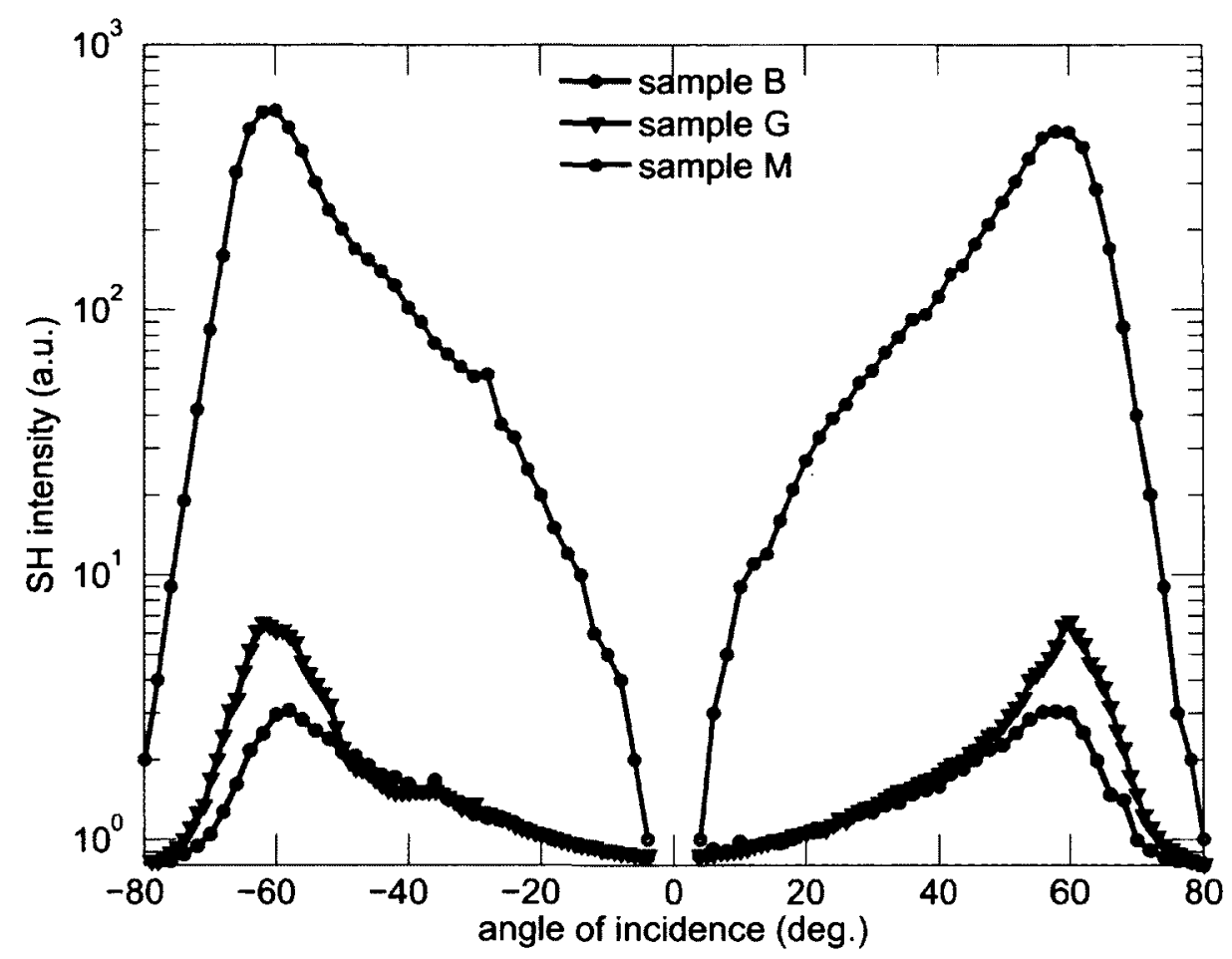

Figure 6.11: Experimentally measured SHG in thermally poled multilayered structures with germanium doped layers shown in Figure 6.10.

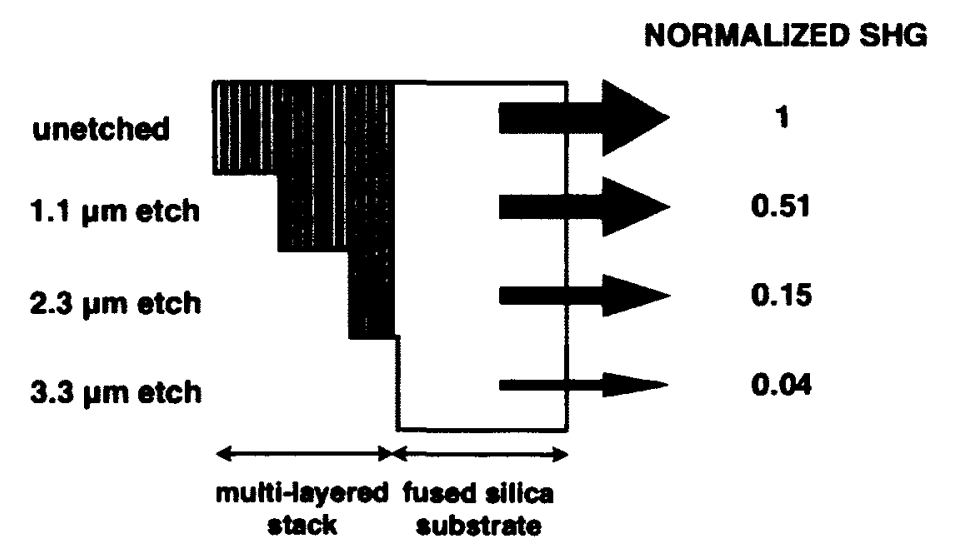

Figure 6.12: Remaining SHG in thermally poled multilayered structure $M$ with a $3 \mu \mathrm{m}$-thick stack after multiple etching experiments.

is expected to offer a tighter control over impurities in the deposited layers than the flame hydrolysis process. Therefore, the poled multilayered structures may lead to an improved reproducibility in the induced nonlinearity compared to synthetic bulk 
glass.

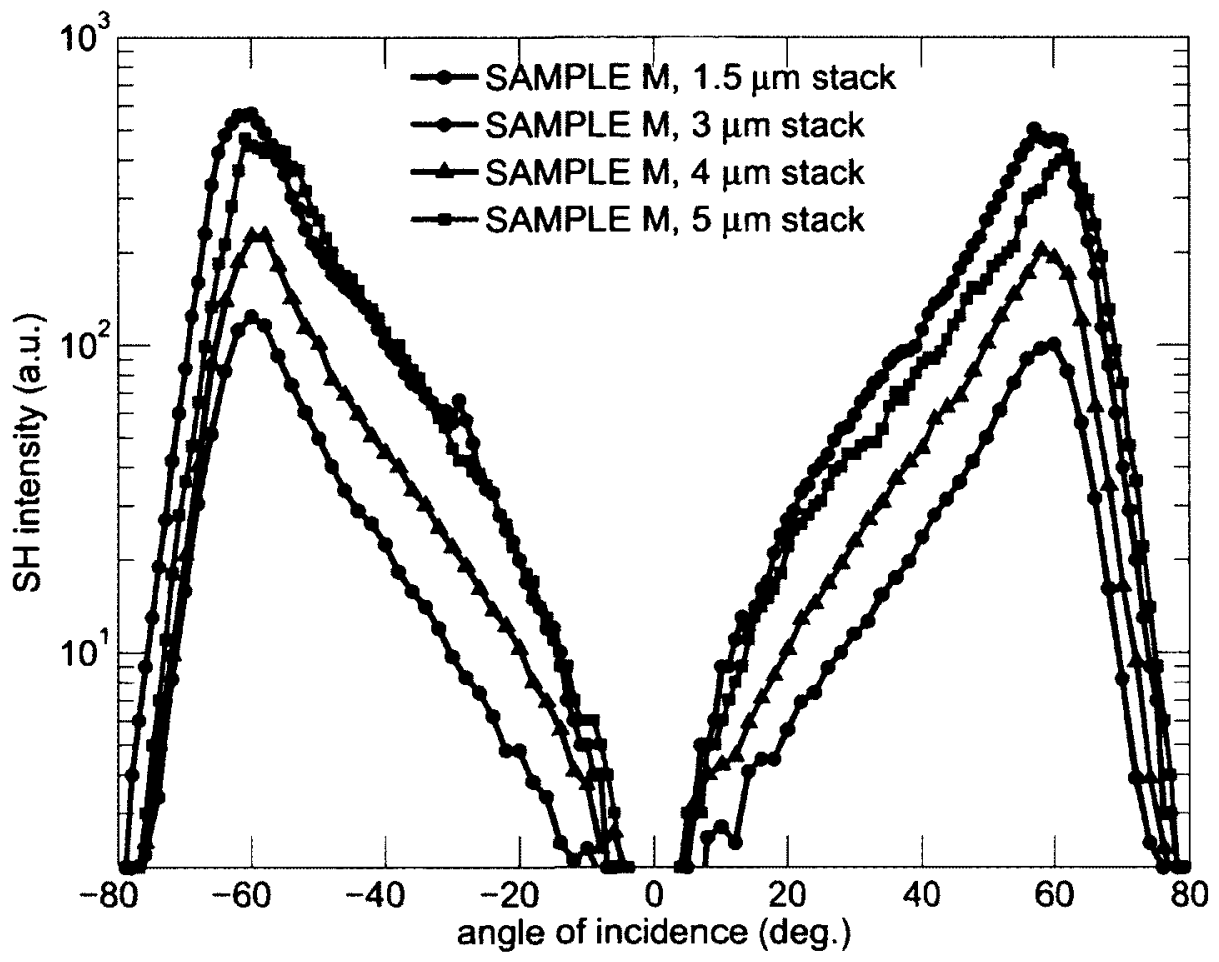

Figure 6.13: Experimentally measured SHG in thermally poled multilayered sample $\mathrm{M}$ with total stack thicknesses ranging from 1.5 to $5 \mu \mathrm{m}$ (with the individual layer thicknesses remaining at $75 \mathrm{~nm}$ for all samples).

The preceding discussion compares the SHG in our multilayered samples with the SHG in Suprasil glass. Although previous reports showed that the SHG in Suprasil is one order of magnitude lower than in other silica glasses [5], it is important to note that the SHG in sample $M$ is still more than an order of magnitude higher than in even the best bulk fused silica glasses. This result clearly indicates that the idea of poled multilayers can be used as a design strategy towards the realization of a thick silica-based nonlinear region. Further improvements in the induced nonlinearity are expected by optimizing design variables such as the layer deposition technique, the parameters of the multilayered stack, and the poling conditions. For instance, we studied variations of sample $M$ with multilayered stacks of varying thicknesses (Figure 6.13), and the results indicate that the large SHG in these structures can be optimized through the design of the multilayered region. 
When germanium is introduced into the silica glass matrix, it substitutes for $\mathrm{Si}^{4+}$ in the $\mathrm{SiO}_{4}$ tetrahedra. Unlike other dopants, germanium incorporates into the lattice without any charge-compensating ions because it has the same number of valence electrons as silicon [158]. The enhanced nonlinearity in samples $\mathrm{G}$ and $\mathrm{M}$ is therefore likely due to a combination of a few factors. First, defects at the interfaces between the germanium-doped and undoped silica act as barriers for the migration of the positive charges injected from the anode, an effect that is consistent with previous observations in poled fibers [131]. These barriers can be advantageous in two ways: (1) the accumulation of alkali impurity ions in the vicinity of the interfaces may create strong localized nonlinearity peaks; or (2) the barriers prevent the detrimental positive ions that are injected from the anode from moving further into the sample. Second, it has been shown experimentally that silica glass with germanium content less than $0.1 \mathrm{~mole} \%$ (as is the case in sample $\mathrm{M}$ ) has a lower ionic conductivity compared to undoped silica [159], further slowing down any positive charges that may have overcome the interface barrier. And third, germanium doping of silica glass increases its refractive index, thus raising its $\chi^{(3)}$ and in turn the induced effective $\chi^{(2)}$. A further increase in the $\chi^{(3)}$ of germanium-doped layers may also occur during the poling process [160].

The two orders of magnitude enhancement in the SHG shown in Figure 6.11 is indeed staggering, especially considering that it was achieved in samples that have not yet been optimized. An important follow-up question is - what is the magnitude of the second-order susceptibility coefficient $\chi^{(2)}$ that is induced in the multilayered stack? Unfortunately, as was explained in section 4.3 , without developing a detailed theoretical model that accounts for pulse propagation effects in ultrafast SHG measurements, this question cannot be answered with the nonlinear characterization setup used in this project. Germanium-doped silica has a prior history of unusually strong nonlinearities [8] [86], which were difficult to explain and reproduce. It would be very interesting to characterize the strongest samples fabricated as part of this project using an alternate method to deduce the value of the induced $\chi^{(2)}$. The results presented here are expected to be easily reproducible because the multilayered structures were deposited using standard microfabrication equipment. Moreover, successful struc- 
tures were replicated in the course of this project with the measured SHG consistent to within the experimental error of the poling process (approximately $\pm 15 \%$ ).

While the precise value of the induced $\chi^{(2)}$ has not been obtained for reasons described above, the relative improvement in the induced nonlinear coefficient in sample M compared to bulk glass can be estimated using equation (2.44). Assuming a step profile for the nonlinearity and using the thicknesses of the nonlinear regions obtained from the etching experiments (46 $\mu \mathrm{m}$ for bulk glass, and $3 \mu \mathrm{m}$ for sample $\mathrm{M}$ ), the ratio $\chi_{\text {sample } M}^{(2)} / \chi_{\text {bulk glass }}^{(2)}$ is calculated to be 7.85 . This substantial increase over the bulk glass nonlinear coefficient may indicate that there is a different mechanism that plays a role during poling of multilayered structures in addition to the frozen-in electric field model that is believed to be responsible for the poling-induced nonlinearity in bulk glass.

Is the large nonlinearity enhancement observed here limited to layers with germanium-doped silica? The results of this chapter suggest that this is not the case. In fact, the comparison of the SHG in samples with micrometer-scale layers (Figure 6.2 and sample G in Figure 6.11) makes it clear that the choice of alternating germaniumdoped and undoped layers does not constitute an optimal structure for poling. Our results suggest that the presence of interfaces is the most important factor, and thus it is likely that an enhancement in the SHG will also be observed in multilayered structures with different dopants. In particular, the introduction of phosphorus-doped layers is expected to increase the induced nonlinearity. Our specific PECVD system was not able to deposit alternating sub-100 nm-thick phosphorus-doped and germaniumdoped layers, but such a structure is easily within reach of other deposition systems. The use of multilayered structures with glasses that have a large intrinsic $\chi^{(3)}$ (for instance, lead glass [15]) may also yield interesting results. Nonetheless, to observe the enhanced effect in germanium-doped glass is encouraging since it is the dominant material for the fabrication of low-loss waveguides and fibers. Moreover, the higher refractive index of germanium-doped films raises the average refractive index of the stack, which creates a planar waveguide structure in which an optical mode could be guided, achieving maximum overlap with the nonlinear region that is localized within the stack. 


\subsection{Summary and Implications}

This chapter presents multilayered design as an effective method for the control of the second-order nonlinearity in thermally poled glass structures. The two most significant results of this chapter are:

1. The dopants used in otherwise identical multilayered structures were shown to affect the magnitude of the induced nonlinearity. This indicates that many design optimizations involving different dopants and glasses are possible. In particular, the presence of phosphorus-doped layers increases the induced nonlinear effect.

2. More than two orders of magnitude improvement was achieved in the SHG in a $3 \mu \mathrm{m}$ multilayered stack consisting of sub-100 nm-thick layers compared to bulk silica glass. This result was demonstrated in a multilayered structure with germanium-doped and undoped layers, which was not yet optimized in terms of the doping levels, layer thicknesses, poling conditions, etc. This indicates that further improvements in the induced nonlinearity are possible and indeed likely.

The approach of subdividing the poled glass into many layers has far-reaching implications for the design of practical silica-based devices with second-order optical nonlinearities. The doped layers can essentially be used as building blocks to create a highly customizable, artificially created nonlinear material. Unlike the case of bulk glass, where the extent and the location of the induced nonlinearity are extremely difficult to control, the multilayered design can be used to induce the nonlinearity precisely within the stack. Although in this thesis only planar structures are considered, the idea of a nonlinear silica glass material can be extended to cylindrical waveguiding structures (optical fibers with a concentric multilayered germanium-doped silica core), where the multilayered design is realized during the fiber preform preparation process. 
Some of the most prominent applications that could benefit from multilayered silica glass nonlinear materials include:

1. All-silica monolithic integration of electro-optic switches and modulators in the PLC platform (as an alternative for the multi-material systems that are in use today).

2. Frequency doubling of rare-earth doped fiber lasers to create rugged and inexpensive lasers in the visible spectral region.

3. Frequency doubling of high-power pulsed lasers (without concern for damage owing to the high optical damage threshold of silica).

4. Realization of an all-fiber monolithic single-photon source for quantum cryptography applications.

The results presented in this chapter give a strong indication that the multilayered approach is the key to overcoming the existing challenges of poled glass devices, thus opening the door to practical implementations of efficient active devices in silica glass. 


\section{Chapter 7}

\section{Conclusion}

A silica-based material with strong second-order nonlinear effects holds the exciting prospects of all-silica monolithic integration of electro-optic devices in planar lightwave circuits and the use of silica as an active medium for frequency conversion applications. In its natural form, silica glass cannot be used as a second-order nonlinear material because of its intrinsic inversion symmetry. Poling techniques can be used to break this symmetry, but research over the past 20 years has not yet resulted in efficient nonlinear interactions in poled glass devices. The limited thickness and the difficulty in controlling the location of the induced nonlinear region result in a poor overlap between the induced nonlinear region and the optical field that interacts with the nonlinearity.

To address this challenge, this doctoral thesis explored the idea of multilayered silica-based design as a method for enhancing the induced nonlinearity in poled silica devices. To the best of our knowledge, this has been the first study specifically targeted at exploring the unique nonlinear effects of multilayered structures during poling. The main hypothesis of this work has been that dopants can be used to control the migration and the distribution of the charges that are responsible for the induced nonlinearity. The results of this thesis confirm this hypothesis, and suggest that artificially created multilayered design may be the key to realizing efficient nonlinear devices in silica. 


\subsection{Summary of Thesis Contributions}

The primary focus of this thesis has been on the experimental investigation of the second-order nonlinear properties in poled silica-based multilayered structures. The main original contributions of this research project are:

\section{Proof-of-concept demonstration of doped silica multilayers as parameters} in the design of nonlinear structures

The question at the center of this thesis has been whether doped silica layers can be designed to control the migration of charges during the poling process. To this extent, multilayered silica structures with phosphorus, boron and germanium doping were investigated. Experiments have shown that the SHG in identical structures with the exception of the dopant used can vary by as much as an order of magnitude. This clearly indicates that the choice of dopants can serve as a parameter in the optimization of the poling process. The effectiveness of the dopants to enhance the poling-induced nonlinearity was related to the way the different dopants incorporate into the silica glass lattice.

\section{Enhancement in the induced second-order nonlinearity in multilayered silica samples}

The results of this thesis conclusively show that multilayered design is a powerful tool for the enhancement of the poling-induced nonlinearity in silica-based devices. As this was the first detailed study of multilayered silica as a nonlinear material, the primary objective was to find the most promising directions for increasing the nonlinearity. A number of variations of multilayered structures were studied for this purpose. However, because of time and equipment restrictions, the structures were not optimized (in terms of deposition methods, dopant composition, layer thickness and configuration, poling conditions, etc.). Yet, these non-optimized structures resulted in very substantial enhancements in the induced effective nonlinearity. It was shown that corona poled multilayered structures with phosphorus-doped and undoped silica resulted in a 
14-fold increase in the SHG. Moreover, in thermal poling experiments, more than two orders of magnitude enhancement in the SHG was obtained in multilayered structures with a large number of sub-100 nm-thick alternating germanium-doped and undoped silica layers.

\section{Experimental demonstration of simultaneous poling of multiple glass layers}

The corona poling experiments presented in Chapter 5 demonstrate that when the poled structure consists of two multilayered stacks separated in space, the stacks exhibit comparable poling-induced nonlinearities. This observation has a profound effect in the broader context of multilayered poled structures. It implies that the multilayered structures can be designed such that they act as multiple charge-accumulating capacitors in series, which divide the applied poling voltage between them such that multiple regions of glass are poled simultaneously. This ability is unique to the multilayered thin-film structures, and is an important step towards enhancing the effective poling-induced nonlinearity by increasing the total thickness over which the nonlinearity occurs.

\section{Evidence of a non-uniform frozen-in electric field tilt in thermally poled multilayered silica samples}

The experiments presented in Chapter 6 indicate that the multilayered structures exhibit a position-dependent angular tilt of the frozen-in electric field that forms during the poling process. In homogeneous bulk glass, mobile charges move away from the anode under the influence of the poling field, and result in a frozen-in field that is always perpendicular to the sample surface. In contrast, in multilayered structures, the migration of the charges is affected not only by the poling field, but also by the multitude of interfaces and/or glass dopants. In addition to charge trapping, there appears to be lateral charge migration that causes position-dependent accumulation of charges within the poled region. This provides a yet unexplored parameter space to engineer nonlinearities that are oriented along directions away from the sample 
normal, thereby allowing optimization of the elements of the nonlinear susceptibility tensor for applications where the incident optical and electrical fields might be constrained by other factors.

This doctoral project resulted in the following publications:

1. K. Yadav, C. W. Smelser, S. Jacob, C. Blanchetiere, C. L. Callender, and J. Albert, "Enhanced second-order nonlinearities in multilayers of nanoscale doped silica thin films," in Bragg Gratings, Photosensitivity, and Poling in Glass Waveguides (BGPP), OSA Technical Digest, paper BTuB6, June 2010.

2. K. Yadav, C. W. Smelser, S. Jacob, C. Blanchetiere, C. L. Callender and J. Albert, "Second-order nonlinearity distribution in a doped silica glass multilayered structure," in Conference on Lasers and Electro-Optics (CLEO), paper CTuL6, May 2011.

3. K. Yadav, C. W. Smelser, S. Jacob, C. Blanchetiere, C. L. Callender, and J. Albert, "Simultaneous corona poling of multiple glass layers for enhanced effective second-order optical nonlinearities," Applied Physics Letters, vol. 99, p. 031109, July 2011.

4. K. Yadav, C. L. Callender, C. W. Smelser, C. Ledderhof, C. Blanchetiere, S. Jacob, and J. Albert, "Giant enhancement of the second harmonic generation efficiency in poled multilayered silica glass structures," Optics Express, vol. 19, p. 26975 26983, December 2011.

\subsection{Recommendations for Future Work}

The substantial enhancement in the induced nonlinearity in the structures studied in this thesis opens up the possibility that silica glass may in fact be engineered to possess strong second-order nonlinear properties over thicknesses compatible with standard waveguide- and fiber-based devices. This area of research is worthy of further 
scientific investigation to gain a better understanding of the underlying mechanisms and to optimize the induced nonlinear effect. The following research directions are recommended for future work on this topic:

\section{Determination of the induced $\chi^{(2)}$}

The primary nonlinear characterization method used in this thesis was the Maker fringe method, where the magnitude of the SHG in the samples was measured and compared. The Maker fringe technique can usually be used to extract the value of the second-order susceptibility coefficient $\chi^{(2)}$ in the sample by comparing the measured SHG to the SHG in a known reference crystal. However, in order to do that in our case, the theoretical Maker fringe model must include pulse propagation effects to account for the short spatial extent of the ultrafast pulses, which was beyond the scope of this thesis. The development of such a model will allow the determination of the induced $\chi^{(2)}$, making a comparison between our experiments and previous work possible, and could provide a direct assessment of the feasibility of multilayered silica for practical devices. It would be particularly interesting to extract the value of the effective $\chi^{(2)}$ in the germanium-doped multilayered samples that exhibit a two orders of magnitude enhancement in the second harmonic signal.

\section{Enhanced nonlinear characterization}

The standard Maker fringe technique measures the cumulative SHG in the sample as a function of incidence angle. However, in the context of multilayers, a much better understanding of the underlying mechanisms could be obtained if not only the cumulative SHG could be measured, but also the variations in the $\chi^{(2)}$ within the multilayered stack. If a longer-pulse laser is used as the source for the Maker fringe measurements, one of the improved Maker fringe characterization methods described in section 3.2 .2 can be used to determine the profile of the induced nonlinearity. Alternatively, a higher-resolution characterization method such as SHG microscopy can also determine the variations in the induced nonlinear coefficient within the multilayered structures. 


\section{Further improvements in the multilayered design}

The use of multilayered design opens the door to many different parameters that can be varied to optimize the induced nonlinearity. A detailed study of the effect of dopant concentration and layer thicknesses is expected to further improve the induced effect. Although in this thesis we focused exclusively on silica glass with standard dopants, other glasses with high $\chi^{(3)}$ may also yield interesting results (e.g. lead or bismuthzinc-borate glasses). Furthermore, the variation in the poling-induced nonlinearity in structures realized by different fabrication methods may point to structural properties of thin films that affect the poling process. In this project, the largest SHG improvement was obtained in structures that consisted of $75 \mathrm{~nm}$-thick layers deposited by PECVD. If further studies indicate that even thinner layers are required for optimal nonlinear effects, other thin film fabrication methods with a slower deposition rate should be considered. It is interesting to note that as the layer thicknesses in these structures approach those of crystals, they could be thought of as artificial crystals with engineered optical and electrical properties. While it is expected that the induced nonlinear effect will increase for multilayered stacks with even thinner layers than those considered in this project, the impact of interdiffision and stress relaxation effects on the lifetime of the nonlinearity in such ultrathin film structures must be investigated. In addition to the optimizations for maximum nonlinearity, other optical properties of the multilayered structures must also be studied and optimized. In particular, low propagation losses are essential if poled multilayered structures are to be used for electro-optic modulation or quasi-phase matched second harmonic generation.

\section{Optimization of the poling conditions}

The experimental poling conditions used in this work were based approximately on the optimal poling conditions reported in literature for bulk glass. The enhanced SHG in the multilayered structures indicates that the layered design affects the distribution and the migration of charges during the poling process. Therefore, it is reasonable to expect that the optimal conditions for the multilayered structures are different from 
those of bulk glass. Studies of the effect of poling voltage, timeline, temperature, atmosphere and electrode configuration are expected to result in further enhancements in the induced nonlinear effect. Moreover, current measurements during the poling process [161] will provide useful information about the dynamics of charge migration in the multilayered structures. Although the study of each of the poling conditions may result in a better understanding of poled multilayers, the investigation of the poling voltage and timeline are believed to be the most promising routes to improve upon the results of this work. It is important to realize that the optimal poling conditions for bulk glass are based on thick layers and charge migration over long distances (tens of micrometers and beyond). In contrast, most the charge migration in the multilayered structures occurs over much shorter distances (at most a few micrometers), and therefore the optimal poling duration is expected to be much shorter than the timeline followed in this project. Moreover, the corona poling results suggest that the multilayered structures act as multiple charge-accumulating capacitors in series. Hence it is likely that the optimal poling voltage in such structures, which is divided between the "capacitors", is higher than that typically used for bulk glass poling.

\section{Investigation of the dielectric strength of the multilayered structures}

Experimental measurements of the profile of the induced nonlinearity presented in Chapter 5 showed that the poling voltage can be divided between layers of glass such that simultaneous poling of multiple glass layers is realized. It is therefore possible that for optimal poling of glass multilayers, one would require a higher poling voltage than the optimal voltage across bulk glass. But what voltage can be applied across the multilayered structure before causing dielectric breakdown? In other words, how does the multilayered stack affect the dielectric strength of the glass structure? Some work has been done to answer these questions, however no conclusion was reached because the experiments required voltages beyond the voltage range of the available equipment. The electric field at which dielectric breakdown occurs depends on many conditions, including the geometries of the dielectric and the electrodes, the rate of the applied field increase, material defects, etc. The performed experiments showed that 
both bulk glass and our multilayered samples can withstand $10 \mathrm{kV}$ across a $0.5 \mathrm{~mm}$ thick sample at $\sim 400{ }^{\circ} \mathrm{C}$ without incurring breakdown as long as: (1) measures are taken to prevent breakdown of air (by using a large sample to increase the pathlength through air, or by utilizing high-dielectric oils or ceramics as insulation around the sample); and (2) the voltage ramp up rate is slow $(\sim 30 \mathrm{~V} / \mathrm{min})$. Due to the limited $10 \mathrm{kV}$ range available on our equipment, the experiments could not continue further to determine whether the dielectric strength of a multilayered structure is higher compared to the strength of bulk glass. For typical poling voltages of $3-10 \mathrm{kV}$ in bulk glass, nearly all the voltage drops across the formed depletion region that may span only a few micrometers, resulting in a frozen-in field that is on the order of the breakdown strength of silica $\left(\sim 10^{9} \mathrm{~V} / \mathrm{m}\right)$. Therefore, dielectric breakdown may become a problem if voltage studies indicate that the optimal poling voltage for the multilayered structures is substantially higher than that for bulk glass.

\section{Spectroscopic characterization of the nonlinear multilayered stack}

Spectroscopic analysis of poled samples may be used to gain a better understanding of the mechanisms responsible for the enhanced poling-induced nonlinear effects in the multilayered structures. Absorption spectroscopy measurements in the visible and near-IR spectral regions were performed on the samples as part of this project. However, no new absorption bands were observed in the multilayered structures compared to silica substrate alone. This is likely due to a very short pathlength within the micrometer-scale layers. Better sensitivity could be achieved if the pathlength is extended (by using multiple reflections or by launching the beam along the deposited layers) or if the background signal is reduced (e.g. by thinning the sample). Alternatively, it may be simpler to directly access the deposited stack without special sample preparations if the measurements are done in reflection. Secondary ion mass spectrometry [111] [112] can also be used to obtain useful information about depletion and accumulation of various species within the induced nonlinear region. 


\section{Study of the effect of stresses on the induced nonlinearity in the multilayered structures}

Interfacial stresses in the multilayered structures may play a role during the poling process due to stress-driven diffusion, a phenomenon where a stress gradient acts as a driving force for diffusion of ions [157]. This hypothesis was pursued as part of this project, however no definite correlation was found between the stresses in the multilayered samples and their poling-induced nonlinearity. This may be due to the fact that the studied wafers were too small (1"-diameter), resulting in inaccurate curvature fitting results in the film stress calculations. The work on the effect of stresses on the poling processes was motivated by a few reports in the literature [112] [15] [162] where film stresses could have accounted for the induced nonlinearity. If larger wafers are used for the stress studies of the multilayered structures, useful insights into the effect of film stresses on the induced nonlinearity may be gained. The results of the stress studies that were performed as part of this project are included in Appendix $\mathrm{A}$ as a reference for future work in this area.

\section{Study of the effect of low-resistivity substrates on the induced nonlinearity in the multilayered stack}

In this project we investigated multilayered stacks deposited on fused silica substrates. However, because the primary interest is in the induced nonlinear effect in the layers (and not the substrate), substrates made of other materials can also be used. In fact, poling of multilayered stacks deposited on substrates with lower resistivity causes most of the poling voltage to drop across the deposited stack [136]. This, in turn, forces the induced nonlinearities to be confined to the multilayered stack such that maximum overlap is achieved between the poling-induced nonlinearity and the guiding region of the multilayered waveguide. Borosilicate glass substrates, whose volume resistivity is five orders of magnitude lower than the resistivity of fused silica, are good candidates if transparency in the visible range is required. Otherwise, similar to the configuration used in planar lightwave circuits, standard silicon substrates can be utilized. 
The results of this doctoral thesis show that multilayered silica-based structures offer a powerful way to enhance and control the poling-induced nonlinearity. As the preceding discussion indicates, the findings presented in this thesis open up new and unexplored avenues for fundamental research on artificially created second-order nonlinear glass materials. Equally important, these findings have multiple important applications, where nonlinear silica-based devices could drastically reduce the cost and complexity of today's optical systems. The experiments presented in this thesis are a successful proof-of-concept demonstration of enhanced second-order nonlinearity in multilayered silica-based materials. They are expected to lead towards further work on this topic that will optimize the induced nonlinearity and result in the development of practical active nonlinear devices in silica. 


\section{Appendix A}

\section{Stress Study of PECVD Multilayered Samples}

For a thin film deposited on a thick circular substrate, the film is subjected to biaxial stress. When the thickness of a film is much smaller than the thickness of the substrate, the radius of curvature of the film / substrate system can be related to the biaxial stress $\sigma_{f}$ in the film by Stoney's equation [163] [164]:

$$
\sigma_{f}=\frac{E_{s}}{1-\nu_{s}} \frac{t_{s}^{2}}{6 t_{f}}\left(\frac{1}{R_{1}}-\frac{1}{R_{2}}\right)
$$

where $E_{s}, \nu_{s}$ and $t_{s}$ are Young's modulus, Poisson's ratio and thickness of the substrate, respectively, $t_{f}$ is the thickness of the film, and $R_{1}$ and $R_{2}$ are the radii of curvature of the substrate before and after the film deposition, respectively. The $E_{s} /\left(1-\nu_{s}\right)$ term represents the biaxial modulus of the substrate material [164]. The above equation assumes that the substrate material is homogeneous, linearly elastic and isotropic, the film material is isotropic, the analysis is done far away from the edges, and the film thickness is much smaller than the substrate thickness, which in turn is much smaller than the lateral dimensions of the structure.

If the stress varies in the thickness direction of the film, equation (A.1) represents an average film stress [165]. For multilayered structures, this equation represents the 
thickness-weighted average of the stresses in all films [166]:

$$
\sigma_{\text {avg }}=\frac{\sigma_{f_{1}} t_{f_{1}}+\sigma_{f_{2}} t_{f_{2}}+\ldots+\sigma_{f_{n}} t_{f_{n}}}{t_{f_{1}}+t_{f_{2}}+\ldots+t_{f_{n}}}
$$

where $\sigma_{f_{1}}, \sigma_{f_{2}}, \ldots \sigma_{f_{n}}$ are the stresses developed in $n$ films of thicknesses $t_{f_{1}}, t_{f_{2}}$, $\ldots t_{f_{n}}$. The above equation is valid as long as the total thickness of the multilayered stack is much smaller than the thickness of the substrate.

The stress study was conducted on multilayered structures deposited by PECVD on 1"-diameter synthetic fused silica substrates and 1"-diameter p-type silicon substrates. Prior to the deposition, the substrate thicknesses were measured by a micrometer, and their initial curvature was measured by a Dektak stylus profiler. The curvature measurement was repeated after the film deposition. In order to obtain the radius of curvatures, the profile scan data was fit to a $5^{\text {th }}$ order polynomial function $y(x)$ using the least squares method (where $x$ is the scanning distance along the substrate), and the radius of curvature was calculated as [167]:

$$
R(x)=\frac{\left[\left(1+\left(\frac{\mathrm{d} y}{\mathrm{~d} x}\right)^{2}\right]^{\frac{3}{2}}\right.}{\frac{\mathrm{d}^{2} y}{\mathrm{~d} x^{2}}}
$$

Table A.1 summarizes the stress results obtained for the deposited multilayered structures with thick layers, while Table A.2 corresponds to the multilayered stacks with many nanolayers. The stresses correspond to unannealed samples, unless indicated otherwise (in which case the samples were annealed for 4 hours at $950{ }^{\circ} \mathrm{C}$ in nitrogen). 


\begin{tabular}{|c|c|c|c|c|}
\hline \multirow{2}{*}{\multicolumn{2}{|c|}{ DEPOSITED STRUCTURE }} & \multirow{2}{*}{$\begin{array}{c}\text { StaCK } \\
\text { ThICKNESS }\end{array}$} & \multicolumn{2}{|c|}{ Average Film Stress (MPa) } \\
\hline & & & $\begin{array}{c}\text { Fused Silica } \\
\text { SubStrate } \\
\end{array}$ & $\begin{array}{c}\text { Silicon } \\
\text { Substrate }\end{array}$ \\
\hline 4 layers: & $\begin{array}{l}\text { undoped silica }(1 \mu \mathrm{m}) \\
\text { undoped silica }(0.2 \mu \mathrm{m}) \\
\text { undoped silica }(1 \mu \mathrm{m}) \\
\text { undoped silica }(0.2 \mu \mathrm{m})\end{array}$ & $2.4 \mu \mathrm{m}$ & -130 & -189 \\
\hline 4 layers: & $\begin{array}{l}\text { undoped silica }(1 \mu \mathrm{m}) \\
\text { P-doped silica }(0.2 \mu \mathrm{m}, 6.1 \mathrm{w} \%) \\
\text { undoped silica }(1 \mu \mathrm{m}) \\
\text { P-doped silica }(0.2 \mu \mathrm{m}, 6.1 \mathrm{w} \%)\end{array}$ & $2.4 \mu \mathrm{m}$ & $\begin{array}{l}\quad-85 \\
\text { (annealed }-37 \text { ) }\end{array}$ & -127 \\
\hline 4 layers: & $\begin{array}{l}\text { undoped silica }(1 \mu \mathrm{m}) \\
\text { P-doped silica }(0.2 \mu \mathrm{m}, 7.6 \mathrm{w} \%) \\
\text { undoped silica }(1 \mu \mathrm{m}) \\
\text { P-doped silica }(0.2 \mu \mathrm{m}, 7.6 \mathrm{w} \%)\end{array}$ & $2.4 \mu \mathrm{m}$ & -74 & -190 \\
\hline 4 layers: & $\begin{array}{l}\text { Ge-doped silica }(1 \mu \mathrm{m}, 22.5 \mathrm{w} \%) \\
\text { undoped silica }(0.2 \mu \mathrm{m}) \\
\text { Ge-doped silica }(1 \mu \mathrm{m}, 22.5 \mathrm{w} \%) \\
\text { undoped silica }(0.2 \mu \mathrm{m})\end{array}$ & $2.4 \mu \mathrm{m}$ & 6 & -63 \\
\hline
\end{tabular}

Table A.1: Average film stresses for multilayered structures with thick layers, deposited on fused silica and silicon substrates (negative values indicate compressive stress, positive values indicate tensile stress).

\begin{tabular}{|c|c|c|c|}
\hline \multirow[b]{2}{*}{ Deposited Structure } & \multirow[b]{2}{*}{$\begin{array}{c}\text { StACK } \\
\text { THICKNESS }\end{array}$} & \multicolumn{2}{|c|}{ Average Film Stress (MPa) } \\
\hline & & $\begin{array}{c}\text { Fused Silica } \\
\text { Substrate }\end{array}$ & $\begin{array}{c}\text { Silicon } \\
\text { SubSTRATE }\end{array}$ \\
\hline $\begin{array}{l}40 \text { alternating layers: } \\
\text { undoped silica }(75 \mathrm{~nm}) \\
\text { Ge-doped silica }(75 \mathrm{~nm}, 0.2 \mathrm{w} \%)\end{array}$ & $3 \mu \mathrm{m}$ & -23 & -238 \\
\hline $\begin{array}{l}54 \text { alternating layers: } \\
\text { undoped silica }(75 \mathrm{~nm}) \\
\text { Ge-doped silica }(75 \mathrm{~nm}, 0.2 \mathrm{w} \%)\end{array}$ & $4 \mu \mathrm{m}$ & -29 & -221 \\
\hline $\begin{array}{l}67 \text { alternating layers: } \\
\text { undoped silica }(75 \mathrm{~nm}) \\
\text { Ge-doped silica }(75 \mathrm{~nm}, 0.2 \mathrm{w} \%)\end{array}$ & $5 \mu \mathrm{m}$ & -33 & -83 \\
\hline
\end{tabular}

Table A.2: Average film stresses for multilayered structures with thin layers, deposited on fused silica and silicon substrates (negative values indicate compressive stress). 


\section{References}

[1] N. Gisin, G. Ribordy, W. Tittel, and H. Zbinden, "Quantum cryptography," Reviews of Modern Physics, vol. 74, pp. 145-195, January 2002.

[2] M. Fiorentino, P. L. Voss, J. E. Sharping, and P. Kumar, "All-fiber photonpair source for quantum communications," IEEE Photonics Technology Letters, vol. 14, pp. 983-985, July 2002.

[3] G. Bonfrate, V. Pruneri, P. G. Kazansky, P. Tapster, and J. G. Rarity, "Parametric fluorescence in periodically poled silica fibers," Applied Physics Letters, vol. 75, pp. 2356-2358, October 1999.

[4] U. Österberg and W. Margulis, "Dye laser pumped by Nd:YAG laser pulses frequency doubled in a glass optical fiber," Optics Letters, vol. 11, no. 8, pp. 516 $518,1986$.

[5] R. A. Myers, N. Mukherjee and S. R. J. Brueck, "Large second-order nonlinearity in poled fused silica," Optics Letters, vol. 16, pp. 1732-1734, November 1991.

[6] A. Okada, K. Ishii, K. Mito and K. Sasaki, "Phase-matched second-harmonic generation in novel corona poled glass waveguides," Applied Physics Letters, vol. 60, pp. 2853-2855, June 1992.

[7] P. Blazkiewicz, W. Xu, D. Wong, J. Canning, M. Åsland, and G. Town, "Carbon dioxide laser-assisted poling of silicate-based optical fibers," Optics Letters, vol. 25, pp. 200-202, February 2000. 
[8] T. Fujiwara, D. Wong, Y. Zhao, S. Fleming, S. Poole, and M. Sceats, "Electrooptic modulation in germanosilicate fibre with UV-excited poling," Electronics Letters, vol. 31, pp. 573-575, March 1995.

[9] C. Corbari, P. G. Kazansky, S. A. Slattery, and D. N. Nikogosyan, "Ultraviolet poling of pure fused silica by high-intensity femtosecond radiation," Applied Physics Letters, vol. 86, p. 071106, February 2005.

[10] P. G. Kazansky, A. Kamal, and P. St. J. Russell, "High second-order nonlinearities induced in lead silicate glass by electron-beam irradiation," Optics Letters, vol. 18, pp. 693-695, May 1993.

[11] L. J. Henry, B. V. McGrath, T. G. Alley, and J. J. Kester, "Optical nonlinearity in fused silica by proton implantation," Journal of the Optical Society of America $B$, vol. 13, pp. 827 836, May 1996.

[12] Y. Quiquempois, P. Niay, M. Douay and B. Poumellec, "Advances in poling and permanently induced phenomena in silica-based glasses," Current Opinion in Solid State \& Materials Science, vol. 7, pp. 89-95, April 2003.

[13] A. Ozcan, M. Digonnet, G. Kino, F. Ay, and A. Aydinli, "Characterization of thermally poled germanosilicate thin films," Optics Express, vol. 12, pp. 4698 4708, October 2004.

[14] J. Arentoft, M. Kristensen, K. Pedersen, S. I. Bozhevolnyi and P. Shi, "Large second-harmonic generation in thermally poled silica waveguides," in Optical Fiber Communication Conference and Exhibit, vol. 1, pp. MC6-1-MC6--3, March 2000.

[15] Y. Luo, A. Biswas, A. Frauenglass and S. R. J. Brueck, "Large second-harmonic signal in thermally poled lead glass-silica waveguides," Applied Physics Letters, vol. 84 , pp. 4935-4937, June 2004. 
[16] H. An and S. Fleming, "Time evolution of the second-order nonlinearity layer in thermally poled optical fiber," Applied Physics Letters, vol. 89, no. 23, pp. 231105-231107, 2006.

[17] B. E. A. Saleh and M. C. Teich, Fundamentals of Photonics. John Wiley \& Sons, second ed., 2007.

[18] P. N. Butcher and D. Cotter, The Elements of Nonlinear Optics. Cambridge University Press, 1990.

[19] I. Asselberghs, J. Perez-Moreno, and K. Clays, "Characterization techniques of nonlinear optical materials," in Non-Linear Optical Properties of Matter (M. G. Papadopoulos, A. J. Sadlej, and J. Leszczynski, ed.), vol. 1 of Challenges and Advances in Computational Chemistry and Physics, pp. 419-459, Springer Netherlands, 2006.

[20] R. F. Shi and A. F. Garito, "Introduction: conventions and standards for nonlinear optical processes," in Characterization techniques and tabulations for organic nonlinear optical materials (M. G. Kuzyk, C. W. Dirk, and M. Dekker, ed.), CRC Press, 1998.

[21] R. L. Sutherland, Handbook of Nonlinear Optics. Marcel Dekker, 2003.

[22] R. W. Boyd, Nonlinear Optics. Academic Press, second ed., 2003.

[23] J. A. Armstrong, N. Bloembergen, J. Ducuing and P. S. Pershan, "Interactions between light waves in a nonlinear dielectric," Physical Review, vol. 127, pp. 1918-1939, September 1962.

[24] A. Yariv and P. Yeh, Photonics - Optical Electronics in Modern Communications. Oxford University Press, Sixth ed., 2007.

[25] J. Jerphagnon and S. K. Kurtz, "Maker fringes: a detailed comparison of theory and experiment for isotropic and uniaxial crystals," Journal of Applied Physics, vol. 41, pp. 1667-1681, March 1970. 
[26] R. Kashyap, Fiber Bragg Gratings. Academic Press, second ed., 2010.

[27] J. E. Midwinter and J. Warner, "The effects of phase matching method and of uniaxial crystal symmetry on the polar distribution of second-order non-linear optical polarization," British Journal of Applied Physics, vol. 16, pp. 1135-1142, August 1965.

[28] M.V. Hobden and J. Warner, "The temperature dependence of the refractive indices of pure lithium niobate," Physics Letters, vol. 22, pp. 243-244, August 1966.

[29] M. M. Fejer, G. A. Magel, D. H. Jundt, and R. L. Byer, "Quasi-phase-matched second harmonic generation: tuning and tolerances," IEEE Journal of Quantum Electronics, vol. 28, pp. 2631-2654, November 1992.

[30] R. Kashyap, G. J. Veldhuis, D. C. Rogers and P. F. Mckee, "Phase-matched second-harmonic generation by periodic poling of fused silica," Applied Physics Letters, vol. 64, pp. 1332-1334, March 1994.

[31] J. M. Dell, M. J. Joyce and G. O. Stone, "Erasure of poling-induced secondorder optical nonlinearities in silica by UV exposure," in Doped Fiber Devices and Systems, vol. 2289, pp. 185-193, July 1994.

[32] K. F. Hulme, "Nonlinear optical crystals and their applications," Reports on Progress in Physics, vol. 36, pp. 497-540, May 1973.

[33] S. K. Kurtz, "Measurement of Nonlinear Optical Susceptibilities," in Quantum Electronics (H. Rabin and C. L. Tang, ed.), Academic Press, 1975.

[34] W. L. Bond, "The mathematics of the physical properties of crystals," The Bell System Technical Journal, vol. 22, pp. 1-72, January 1943.

[35] J. F. Nye, Physical Properties of Crystals, Their Representation by Tensors and Matrices. Oxford, 1960. 
[36] P. D. Maker, R. W. Terhune, M. Nisenoff and C. M. Savage, "Effects of dispersion and focusing on the production of optical harmonics," Physical Review Letters, vol. 8, pp. 21-22, January 1962.

[37] I. Shoji, T. Kondo, A. Kitamoto, M. Shirane, and R. Ito, "Absolute scale of second-order nonlinear-optical coefficients," Journal of the Optical Society of America, vol. 14, pp. 2268-2294, September 1997.

[38] M. J. Weber, Handbook of Optical Materials. CRC Press, 2003.

[39] W. J. Alford and A. V. Smith, "Wavelength variation of the second-order nonlinear coefficients of $\mathrm{KNbO}_{3}, \mathrm{KTiOPO}_{4}, \mathrm{KTiOAsO}_{4}, \mathrm{LiNbO}_{3}, \mathrm{LiIO}_{3}, \beta-\mathrm{BaB}_{2} \mathrm{O}_{4}$, $\mathrm{KH}_{2} \mathrm{PO}_{4}$, and $\mathrm{LiB}_{3} \mathrm{O}_{5}$ crystals: a test of Miller wavelength scaling," Journal of the Optical Society of America B, vol. 18, pp. 524-533, April 2001.

[40] V. G. Dmitriev, G. G. Gurzadyan, and D. N. Nikogosyan, Handbook of Nonlinear Optical Crystals. Springer-Verlag, 1991.

[41] R. C. Miller, "Optical second harmonic generation in piezo-electric crystals," Applied Physics Letters, vol. 5, pp. 17--19, July 1964.

[42] C. G. B. Garrett and F. N. H. Robinson, "Miller's phenomenological rule for computing nonlinear susceptibilities," IEEE Journal of Quantum Electronics, vol. QE-2, pp. 328-329, 1966.

[43] A. Yariv and P. Yeh, Optical Waves in Crystals - Propagation and Control of Laser Radiation. Wiley-Interscience, 2003.

[44] R. C. Alferness, "Waveguide electrooptic modulators," in IEEE Transactions on Microwave Theory and Techniques, vol. 30, pp. 1121-1137, August 1981.

[45] R. C. Alferness, S. K. Korotky, and E. A. J Marcatili, "Velocity-matching techniques for integrated optic traveling wave switch/modulators," IEEE Journal of Quantum Electronics, vol. QE-20, pp. 301-309, March 1984. 
[46] E. L. Wooten, K. M. Kissa, A. Yi-Yan, E. J. Murphy, D. A. Lafaw, P. F. Hallemeier, D. Maack, D. V. Attanasio, D. J. Fritz, G. J. McBrien, and D. E. Bossi, "A review of lithium niobate modulators for fiber-optic communications systems," IEEE Journal of Selected Topics in Quantum Electronics, vol. 6, pp. 69-82, January 2000.

[47] W. S. C. Chang, RF Photonic Technology in Optical Fiber Links. Cambridge University Press, 2002.

[48] D. Marcuse, Theory of dielectric optical waveguides. Academic Press, 1974.

[49] C. M. Kim and R. V. Ramaswamy, "Overlap integral factors in integrated optic modulators and switches," Journal of Lightwave Technology, vol. 7, pp. 1063 1070, July 1989.

[50] S. McMeekin, R. M. De La Rue, and W. Johnstone, "The transverse electrooptic modulator (TEOM): fabrication, properties, and applications in the assessment of waveguide electrooptic characteristics," Journal of Lightwave Technology, vol. 10, pp. 163-168, February 1992.

[51] D. Janner, D. Tulli, M. Belmonte, and V. Pruneri, "Waveguide electro-optic modulation in micro-engineered $\mathrm{LiNbO}_{3}$, " Journal of Optics A: Pure and Applied Optics, vol. 10, p. 104003, October 2008.

[52] Y. Fujii, B. S. Kawasaki, K. O. Hill, and D. C. Johnson, "Sum-frequency light generation in optical fibers," Optics Letters, vol. 5, pp. 48-50, February 1980.

[53] E. M. Dianov, P. G. Kazanskii, and D. Y. Stepanov, "Problem of the photoinduced second harmonic generation in optical fibers," Soviet Journal of Quantum Electronics, vol. 19, pp. 575-576, May 1989.

[54] E. E. Dianov, P. G. Kazansky, and D. Y. Stephanov, "Photovoltaic model of photoinduced second harmonic generation in optical fibers," Soviet Lightwave Communications, vol. 1, pp. 247-253, August 1991. 
[55] R. H. Stolen and H. W. K. Tom, "Self-organized phase-matched harmonic generation in optical fibers," Optics Letters, vol. 12, pp. 585-587, August 1987.

[56] P. G. Kazansky, A. Kamal and P. St. J. Russell, "Erasure of thermally poled second-order nonlinearity in fused silica by electron implantation," Optics Letters, vol. 18, pp. 1141-1143, July 1992.

[57] W. Margulis and F. Laurell, "Interferometric study of poled glass under etching," Optics Letters, vol. 21, pp. 1786-1788, November 1996.

[58] D. Pureur, A. C. Liu, M. J. F. Digonnet, and G. S. Kin, "Absolute measurement of the second-order nonlinearity profile in poled silica," Optics Letters, vol. 23, pp. 588-590, April 1998.

[59] D. E. Carlson, "Ion depletion of glass at a blocking anode: I, theory and experimental results for alkali silicate glasses," Journal of the American Ceramic Society, vol. 57, pp. 291-294, July 1974.

[60] P. G. Kazansky, A. R. Smith, P. St. J. Russell, G. M. Yang and G. M. Sessler, "Thermally poled silica glass: Laser induced pressure pulse probe of charge distribution," Applied Physics Letters, vol. 68, pp. 269-271, January 1996.

[61] T. G. Alley and S. R. J. Brueck, "Visualization of the nonlinear optical spacecharge region of bulk thermally poled fused-silica glass," Optics Letters, vol. 23, pp. 1170-1172, August 1998.

[62] Y. Quiquempois, N. Godbout and S. Lacroix, "Model of charge migration during thermal poling in silica glasses: Evidence of a voltage threshold for the onset of a second-order nonlinearity," Physical Review A, vol. 65, pp. 043816-1-04381614, April 2002.

[63] M. Dussauze, V. Rodriguez, A. Lipovskii, M. Petrov, C. Smith, K. Richardson, T. Cardinal, E. Fargin, and E. I. Kamitsos, "How does thermal poling affect the structure of soda-lime glass?," The Journal of Physical Chemistry C, vol. 114, pp. 12754-12759, July 2010. 
[64] P. G. Kazansky and P. St. J. Russell, "Thermally poled glass: frozen-in electric field or oriented dipoles?," Optics Communications, vol. 110, pp. 611-614, September 1994.

[65] N. Mukherjee, R. A. Myers and S. R. J. Brueck, "Dynamics of second-harmonic generation in fused silica," Journal of the Optical Society of America B, vol. 11, pp. 665-669, April 1994.

[66] F. C. Garcia, I. C. S. Carvalho, E. Hering, W. Margulis and B. Lesche, "Inducing a large second-order optical nonlinearity in soft glasses by poling," Applied Physics Letters, vol. 72, pp. 3252-3254, June 1998.

[67] H. Guillet de Chatellus, S. Montant, and E. Freysz, "Nondestructive method for characterization of the second-order nonlinear profile and charge distribution in thermally poled fused silica," Optics Letters, vol. 25, pp. 1723-1725, December 2000 .

[68] E. Y. Zhu, L. Qian, L. G. Helt, M. Liscidini, J. E. Sipe, C. Corbari, A. Canagasabey, M. Ibsen, and P. G. Kazansky, "Measurement of $\chi^{(2)}$ symmetry in a poled fiber," Optics Letters, vol. 35, pp. 1530-1532, May 2010.

[69] R. W. Boyd, Nonlinear Optics. Academic Press, third ed., 2007.

[70] R. Adair, L. L. Chase and S. A. Payne, "Nonlinear refractive-index measurements of glasses using three-wave frequency mixing," Journal of the Optical Society of America B, vol. 4, pp. 875-, June 1987.

[71] B. Buchalter and G. R. Meredith, "Third-order optical susceptibility of glasses determined by third harmonic generation," Applied Optics, vol. 21, pp. 32213224, September 1982.

[72] A. Le Calvez, E. Freysz and A. Ducasse, "Experimental study of the origin of the second-order nonlinearities induced in thermally poled fused silica," Optics Letters, vol. 22, pp. 1547-1549, October 1997. 
[73] P. G. Kazansky, L. Dong, P. S. J. Russell, "High second-order nonlinearities in poled silicate fibers," Optics Letters, vol. 19, pp. 701-703, May 1994.

[74] H. An and S. Fleming, "Characterization of a second-order nonlinear layer profile in thermally poled optical fibers with second-harmonic microscopy," Optics Letters, vol. 30, pp. 866-868, April 2005.

[75] A. De Francesco and G. E. Town, "Modeling the electrooptic evolution in thermally poled germanosilicate fibers," IEEE Journal of Quantum Electronics, vol. 37 , pp. 1312-1320, October 2001.

[76] A. Canagasabey, C. Corbari, Z. Zhang, P. G. Kazansky, and M. Ibsen, "Broadly tunable second-harmonic generation in periodically poled silica fibers," Optics Letters, vol. 32, pp. 1863-1865, July 2007.

[77] A. Canagasabey, C. Corbari, A. V. Gladyshev, F. Liegeois, S. Guillemet, Y. Hernandez, M. V. Yashkov, A. Kosolapov, E. M. Dianov, M. Ibsen, and P. G. Kazansky, "High-average-power second-harmonic generation from periodically poled silica fibers," Optics Letters, vol. 34, pp. 2483-2485, August 2009.

[78] E. Y. Zhu, L. Qian, L. G. Helt, M. Liscidini, J. E. Sipe, C. Corbari, A. Canagasabey, M. Ibsen, and P. G. Kazansky, "Phase-matching with a twist: Secondharmonic generation in birefringent periodically poled fibers," Journal of the Optical Society of America B, vol. 27, pp. 2410-2415, November 2010.

[79] A. Canagasabey, M. Ibsen, K. Gallo, A. V. Gladyshev, E. M. Dianov, C. Corbari, and P. G. Kazansky, "Aperiodically poled silica fibers for bandwidth control of quasi-phase-matched second-harmonic generation," Optics Letters, vol. 35, pp. 724-726, March 2010.

[80] H. Imai, S. Horinouchi, Y. Uchida, H. Yamasaki, K. Fukao, G. Zhang, T. Kinoshita, K. Mito, H. Hirashima and K. Sasaki, "Time-dependent decay of quadratic non-linearity in corona-poled silicate glass films," Journal of NonCrystalline Solids, vol. 196, pp. 63-66, March 1996. 
[81] A. Okada, K. Ishii, K. Mito and K. Sasaki, "Second-order optical nonlinearity in corona-poled glass films," Journal of Applied Physics, vol. 74, pp. 531-535, July 1993.

[82] S. Horinouchi, G.J. Zhang, T. Kinoshita, K. Mito and K. Sasaki, "Second harmonic generation in corona-poled glass film waveguides," Nonlinear Optics, vol. 14, pp. 301-312, 1995.

[83] Z. Xu, L. Liu, Z. Hou, P. Yang, X. Liu, L. Xu, W. Wang, M Affatigato and S. Feller, "Influence of different poling methods on the second-order nonlinearity in fused silica glasses," Optics Communications, vol. 174, pp. 475-479, February 2000 .

[84] Y. Quiquempois, G. Martinelli, P. Bernage, M. Douay, P. Niay, E. Delevaque, H. Poignant, B. Loisel, J. F. Bayon, "Study of organized $\chi^{(2)}$ susceptibility in germanosilicate optical fibers," Optical Materials, vol. 9, pp. 361-367, January 1998.

[85] W. Xu, P. Blazkiewicz, and S. Fleming, "Silica fiber poling technology," $A d$ vanced Materials, vol. 13, pp. 1014-1018, July 2001.

[86] T. Fujiwara, M. Takahashi, and A. J. Ikushima, "Second-harmonic generation in germanosilicate glass poled with ArF laser irradiation," Applied Physics Letters, vol. 71, pp. 1032-1034, August 1997.

[87] T. Fujiwara, S. Matsumoto, M. Ohama, and A. J. Ikushima, "Origin and properties of second-order optical non-linearity in ultraviolet-poled $\mathrm{GeO}_{2} \mathrm{SiO}_{2}$ glass," Journal of Non-Crystalline Solids, vol. 273, pp. 203-208, August 2000.

[88] H. An and S. Fleming, "Creating large second-order nonlinearity in twin-hole optical fibre with core at the centre of the two holes," Electronics Letters, vol. 43, pp. 206-207, February 2007. 
[89] M. Qiu, F. Pi and G. Orriols, "The role of lead component in second-harmonic generation in lead silica by electron-beam irradiation," Applied Physics Letters, vol. 73, pp. 3040 -3042, November 1998.

[90] A. Kameyama, E. Muroi, A. Yokotani, K. Kurosawa, and P. R. Herman, "X-ray radiation effects on second-harmonic generation in thermally poled silica glass," Joumal of the Optical Society of America B, vol. 14, pp. 1088-1092, May 1997.

[91] K. Tanaka, K. Kashima, H. Kazuyuki, S. Naohiro, S. Yamagata, A. Mito, and H. Nasu, "Effect of $\gamma$-irradiation on optical second harmonic intensity of electrically poled silica glass," Japanese Jouranl of Applied Physics, vol. 34, pp. 173-174, January 1995.

[92] B. Lesche, F. C. Garcia, E. N. Hering, W. Margulis, I. C. S. Carvalho, and F. Laurell, "Etching of silica glass under electric fields," Physical Review Letters, vol. 78, pp. 2172-2175, March 1997.

[93] A. L. C. Triques, I. C. S. Carvalho, M. F. Moreira, H. R. Carvalho, and R. Fischer, B. Lesche and W. Margulis, "Time evolution of depletion region in poled silica," Applied Physics Letters, vol. 82, pp. 2948 2950, May 2003.

[94] A. Kudlinski, Y. Quiquempois, M. Lelek, H. Zeghlache and G. Martinelli, "Complete characterization of the nonlinear spatial distribution induced in poled silica glass with a submicron resolution," Applied Physics Letters, vol. 83, pp. 3623 3625 , October 2003.

[95] A. Kudlinski, G. Martinelli and Y. Quiquempois, "Time evolution of secondorder nonlinear profiles induced within thermally poled silica samples," Optics Letters, vol. 30, pp. 1039-1041, May 2005.

[96] A. Kudlinski, Y. Quiquempois and G. Martinelli, "Modeling of the $\chi^{(2)}$ susceptibility time-evolution in thermally poled fused silica," Optics Express, vol. 13, pp. 8015-8024, October 2005. 
[97] Y. Quiquempois, G. Martinelli, F. Valentin, P. Bernage, P. Nilay, and M. Douay, "Improved method for measuring second-order non-linearity profile in poled silica," in Bragg Gratings, Photosensitivity, and Poling in Glass Waveguides (BGPP), OSA Technical Digest, vol. 33, pp. 404-410, September 1999.

[98] A. Ozcan, M. J. F. Digonnet, and G. S. Kino, "Cylinder-assisted Maker-fringe technique," Electronics Letters, vol. 39, no. 25, pp. 1834-1836, 2003.

[99] Y. Quiquempois, G. Martinelli, P. Duthérage, P. Bernage, P. Niay and M. Douay, "Localisation of the induced second-order non-linearity within Infrasil and Suprasil thermally poled glasses," Optics Communications, vol. 176, pp. 479-487, April 2000.

[100] Heraeus, Quartz Glass for Optics - Data and Properties, May 2007.

[101] A. C. Liu, M. J. F. Digonnet, G. S. Kino, and E. J. Knystautas, "Advances in the measurement of the poled silica nonlinear profile," in Proceedings of SPIE (Doped Fiber Devices), vol. 3542, pp. 115-119, December 1998.

[102] C. Corbari, O. Deparis, B. G. Klappauf, and P. G. Kazansky, "Practical technique for measurement of second-order nonlinearity in poled glass," Electronics Letters, vol. 39, pp. 197-198, January 2003.

[103] A. Ozcan, M. J. F. Digonnet, and G. S. Kino, "Inverse Fourier transform technique to determine second-order optical nonlinearity spatial profiles," Applied Physics Letters, vol. 82, pp. 1362-1364, March 2003.

[104] V. Treanton, N. Godbout, and S. Lacroix, "Nondestructive interferometric determination of $(2)(\mathrm{z})$ spatial distribution induced in thermally poled silica glasses," Journal of the Optical Society of America B, vol. 21, pp. 2213-2220, December 2004.

[105] A. Ozcan, M. Digonnet, and G. Kino, "Iterative processing of second-order optical nonlinearity depth profiles," Optics Express, vol. 12, pp. 3367-3376, July 2004. 
[106] D. Faccio, V. Pruneri and P. G. Kazansky, "Noncollinear Maker's fringe measurements of second-order nonlinear optical layers," Optics Letters, vol. 25, pp. 1376-1378, September 2000.

[107] H. An, S. Fleming, and G. Cox, "Visualization of second-order nonlinear layer in thermally poled fused silica glass," Applied Physics Letters, vol. 85, pp. 5819 5821, December 2004.

[108] K. Pedersen, S. I. Bozhevolnyi, J. Arentoft, M. Kristensen and C. LaurentLund, "Second-harmonic scanning optical microscopy of poled silica waveguides," Journal of Applied Physics, vol. 88, pp. 3872 3878, October 2000.

[109] J. Arentoft, K. Pedersen, S. I. Bozhevolnyi, M. Kristensen, P. Yu and C. B. Nielsen, "Second-harmonic imaging of poled silica waveguides," Applied Physics Letters, vol. 76, pp. 25-27, January 2000.

[110] C. M. Lepienski, J. A. Giacometti, G. F. Leal Ferreira, F. L. Freire Jr., and C. A. Achete, "Electric field distribution and near-surface modifications in soda-lime glass submitted to a dc potential," Journal of Non-Crystalline Solids, vol. 159, no. 3, pp. $204-212,1993$.

[111] T. G. Alley, S. R. J. Brueck and M. Wiedenbeck, "Secondary ion mass spectrometry study of space-charge formation in thermally poled fused silica," Journal of Applied Physics, vol. 86, pp. 6634-6640, December 1999.

[112] J. Arentoft, M. Kristensen, K. Pedersen, S. I. Bozhevolnyi, and P. Shi, "Poling of silica with silver-containing electrodes," Electronics Letters, vol. 36, pp. 16351636, September 2000.

[113] H. Guillet de Chatellus and E. Freysz, "Static and dynamic profile of the electric field within the bulk of fused silica glass during and after thermal poling," Optics Letters, vol. 28, pp. 1624-1626, September 2003. 
[114] A. Le Calvez, E. Freysz and A. Ducasse, "A model for second harmonic generation in poled glasses," The European Physical Journal D, vol. 1, pp. 223 226, February 1998.

[115] J. Fage-Pedersen, R. Jacobsen and M. Kristensen, "Poled-glass devices: influence of surfaces and interfaces," Journal of the Optical Society of America B, vol. 24, pp. 1075-1079, May 2007.

[116] D. Yudistira, D. Faccio, C. Corbari, P. G. Kazansky, S. Benchabane, and V. Pruneri, "Electric surface potential and frozen-in field direct measurements in thermally poled silica," Applied Physics Letters, vol. 92, pp. 012912-1-0129123 , January 2008.

[117] A. C. Liu, M. J. F. Digonnet and G. S. Kino, "Electro-optic phase modulation in a silica channel waveguide," Optics Letters, vol. 19, pp. 466-468, April 1994.

[118] X.-C. Long, R. A. Myers, and S.R. J. Brueck, "Measurement of the linear electro-optic coefficient in poled amorphous silica," Optics Letters, vol. 19, pp. 1819-1821, November 1994.

[119] M. Abe, T. Kitagawa, K. Hattori, A. Himeno, and Y. Ohmori, "Electro-optic switch constructed with a poled silica-based waveguide on a Si substrate," Electronics Letters, vol. 32, pp. 893-894, May 1996.

[120] X.-C. Long, R. A. Myers and S. R. J. Brueck, "Measurement of linear electrooptic effect in temperature/electric-field poled optical fibres," Electronics Letters, vol. 30, pp. 2162-2163, December 1994.

[121] X.-C. Long, R. A. Myers, and S. R. J. Brueck;, "A poled electrooptic fiber," IEEE Photonics Technology Letters, vol. 8, pp. 227-229, February 1996.

[122] X.-C. Long and S.R.J. Brueck, "Large-signal phase retardation with a poled electrooptic fiber," IEEE Photonics Technology Letters, vol. 9, pp. 767-769, June 1997. 
[123] W. Xu, J. Arentoft, D. Wong and S. Fleming, "Evidence of space-charge effects in thermal poling," IEEE Photonics Technology Letters, vol. 11, pp. 1265-1267, October 1999.

[124] M. Janos, W. Xu, D. Wong, H. Inglis, and S. Fleming, "Growth and decay of the electrooptic effect in thermally poled B/Ge codoped fiber," Journal of Lightwave Technology, vol. 17, pp. 1037-1041, June 1999.

[125] W. Xu, P. Blazkiewicz, D. Wong, S. Fleming, and T. Ryan, "Specialty optical fibre for stabilising and enhancing electro-optic effect induced by poling," Electronics Letters, vol. 36, pp. 1265-1266, July 2000.

[126] A. M. Michie, A. Argyros, S. Fleming, H. An, J. Haywood, and M. Matar, "Multi-wavelength interrogation (MWI) of thermally poled twin-hole silica optical fibres," in Bragg Gratings, Photosensitivity, and Poling in Glass Waveguides (BGPP), OSA Technical Digest, p. BTuB8, June 2010.

[127] M. Fokine, L. E. Nilsson, A. Claesson, D. Berlemont, L. Kjellberg, L. Krummenacher, and W. Margulis, "Integrated fiber Mach-Zehnder interferometer for electro-optic switching," Optics Letters, vol. 27, pp. 1643-1645, September 2002 .

[128] N. Myren, H. Olsson, L. Norin, N. Sjodin, P. Helander, J. Svennebrink, and W. Margulis, "Wide wedge-shaped depletion region in thermally poled fiber with alloy electrodes," Optics Express, vol. 12, pp. 6093 6099, December 2004.

[129] K. Lee, P. Hu, J. L. Blows, D. Thorncraft, and J. Baxter, "200-m optical fiber with an integrated electrode and its poling," Optics Letters, vol. 29, pp. 21242126, September 2004 .

[130] D. Faccio, A. Busacca, W. Belardi, V. Pruneri, P. G. Kazansky, T. M. Monro, D. J. Richardson, B. Grappe, M. Cooper, and C. N. Pannell, "Demonstration of thermal poling in holey fibres," Electronics Letters, vol. 37, pp. 107-108, January 2001. 
[131] H. An and S. Fleming, "Hindering effect of the core-cladding interface on the progression of the second-order nonlinearity layer in thermally poled optical fibers," Applied Physics Letters, vol. 87, pp. 101108-1-3, August 2005.

[132] D. Faccio, A. Busacca, D. W. J. Harwood, G. Bonfrate, V. Pruneri and P. G. Kazansky, "Effect of core-cladding interface on thermal poling of germanosilicate optical waveguides," Optics Communications, vol. 196, pp. 187-190, September 2001.

[133] H. An and S. Fleming, "Characterisation of thermally poled multilayered silicate thin films," in Opto-Electronics and Communications Conference, pp. 1-2, July 2008.

[134] A. Kudlinski, G. Martinelli and Y. Quiquempois, "Dynamics of the secondorder nonlinearity induced in Suprasil glass thermally poled with continuous and alternating fields," Journal of Applied Physics, vol. 103, pp. 063109-1063109 6, March 2008.

[135] M. Fokine, M. Ferraris and I. C. Carvalho, "Thermal poling of glass: a nonlinear ionic RC circuit," in Bragg Gratings, Photosensitivity, and Poling in Glass Waveguides (BGPP), OSA Technical Digest, p. JWBPDP6, September 2007.

[136] C. Corbari, L.C. Ajitdoss, I.C.S. Carvalho, O. Deparis, F.P. Mezzapesa, P.G. Kazansky, K. Sakaguchi, "The problem of achieving high second-order nonlinearities in glasses: The role of electronic conductivity in poling of high index glasses," Journal of Non-Crystalline Solids, 2010.

[137] A. Densmore, S. Janz, R. Ma, J. H. Schmid, D.-X. Xu, A. Delage, J. Lapointe, M. Vachon, and P. Cheben, "Compact and low power thermo-optic switch using folded silicon waveguides," Optics Express, vol. 17, pp. 10457-10465, June 2009.

[138] K. Iniewski, C. McCrosky, and D. Minoli, Network Infrastructure and Architecture: Designing High-Availability Networks. Wiley-Interscience, 2008. 
[139] S. Fevrier, R. Jamier J.-M. Blondy, S. L. Semjonov, M. E. Likhachev, M. M. Bubnov, E. M. Dianov, V. F. Khopin, M. Y. Salganskii, A. N. Guryanov, "Lowloss singlemode large mode area all-silica photonic bandgap fiber," Optics Express, vol. 14, pp. 562-569, January 2006.

[140] Tosoh SGM Corporation, Synthetic Silica Glass ES Grades, 2008.

[141] A. Kudlinski, G. Martinelli and Y. Quiquempois, "Influence of electrode composition on the second-order nonlinearity prole in thermally poled silica glass," Optics Letters, vol. 32, pp. 1773-1775, July 2007.

[142] H. Takebe, P. G. Kazansky, P. St. J. Russell and K. Morinaga, "Effect of poling conditions on second-harmonic generation in fused silica," Optics Letters, vol. 21, pp. 468-470, April 1996.

[143] J. Xu, X. Lu, H. Chen, L. Liu, W. Wang, C. Zhu, and F. Gan, "Second harmonic generation investigation on electric poling effects in fused silica," Optical Materials, vol. 8, pp. 243-247, November 1997.

[144] W. E. Angerer, N. Yang, A. G. Yodh, M. A. Khan, and C. J. Sun, "Ultrafast second-harmonic generation spectroscopy of GaN thin films on sapphire," Physical Review B (Condensed Matter and Materials Physics), vol. 59, pp. 2932-2946, January 1999.

[145] M. A. Mortazavi, A. Knoesen, S. T. Kowel, B. G. Higgins, and A. Dienes, "Second-harmonic generation and absorption studies of polymerdye films oriented by corona-onset poling at elevated temperatures," Journal of the Optical Society of America B, vol. 6, pp. 733-741, April 1989.

[146] S. Horinouchi, H. Imai, G. J. Zhang, K. Mito and K. Sasaki, "Optical quadratic nonlinearity in multilayer corona-poled glass films," Applied Physics Letters, vol. 68 , pp. $3552-3554$, June 1996. 
[147] R. Comizzoli, "Corona discharge - electrostatic method for deposition of powdered passivation glass on semiconductor devices," in IEEE Transactions on Parts, Hybrids, and Packaging, vol. 13, pp. 322 328, September 1977.

[148] S. Franssila, Introduction to Microfabrication. John Wiley \& Sons, 2004.

[149] J. M. Eldridge and D. R. Kerr, "Sodium ion drift through phosphosilicate glass$\mathrm{SiO}_{2}$ films," Journal of the Electrochemical Society, vol. 118, pp. 986-991, June 1971.

[150] D. Shin, S. K. Lee, W. H. Ryu and Y.-C. Chung, "Computer simulation of the thermal poling process inducing second-order optical nonlinearity in silica glass," Journal of the Korean Physical Society, vol. 42, pp. 499-504, April 2003.

[151] H. An and S. Fleming, "Second-order optical nonlinearity and accompanying near-surface structural modifications in thermally poled soda-lime silicate glasses," Journal of the Optical Society of America B, vol. 23, pp. 2303-2309, November 2006.

[152] V. I. Gavrilenko, Optics of Nanomaterials. Pan Stanford Publishing, 2010.

[153] P. S. Russell, L. J. Poyntz-Wright, and D. P. Hand, "Frequency doubling, absorption, and grating formation in glass fibers: effective defects or defective effects?," in Proceedings of SPIE, vol. 1373, pp. 126-139, 1991.

[154] F. Ay, A. Aydinlia, and S. Agan, "Low-loss as-grown germanosilicate layers for optical waveguides," Applied Physics Letters, vol. 83, pp. 4743-4745, December 2003.

[155] S. Cheon, H. Lee, J.-H. Choi, and M. Cho, "Doubly resonant three-wave-mixing spectroscopy of a chiral coupled-chromophore system in solution: Coherent twodimensional optical activity spectroscopy," The Journal of Chemical Physics, vol. 126, pp. 054505-1-14, February 2007.

[156] D. A. Kleinman, "Nonlinear dielectric polarization in optical media," Physical Review, vol. 126, pp. 1977-1979, June 1962. 
[157] F. Célarié, M. Ciccotti and C. Marlière, "Stress-enhanced ion diffusion at the vicinity of a crack tip as evidenced by atomic force microscopy in silicate glasses," Journal of Non-Crystalline Solids, vol. 353, pp. 51-68, January 2007.

[158] D. L. Griscom, "Trapped-electron centers in pure and doped glassy silica: A review and synthesis," Journal of Non-Crystalline Solids, vol. 357, pp. 19451962, April 2011.

[159] A. Feltri, S. Grandi, P. Mustarelli, M. Cutroni, and A. Mandanici, "GeO2doped silica glasses: an ac conductivity study," Solid State Ionics, vol. 154-155, pp. 217-221, December 2002.

[160] F. C. Garcia, L. Vogelaar and R. Kashyap, "Poling of a channel waveguide," Optics Express, vol. 11, pp. 3041-3047, November 2003.

[161] N. Godbout, S. Lacroix, "Characterization of thermal poling in silica glasses by current measurements," Journal of Non-Crystalline Solids, vol. 316, pp. 338348, February 2003.

[162] M. Fokine, K. Saito and A. J. Ikushima, "Thermally induced second-order nonlinearity in silica-based glasses," Applied Physics Letters, vol. 87, no. 17, pp. $171907-1-3,2005$.

[163] G. G. Stoney, "The tension of metallic films deposited by electrolysis," Proceedings of the Royal Society of London A, vol. 82, pp. 172-175, May 1909.

[164] R. W. Hoffman, Physics of Thin Films, vol. 3. Academic Press, 1966.

[165] S. P. Baker and W. D. Nix, "Mechanical properties of thin films on substrates," in Proceedings of SPIE, vol. 1323, pp. 263-276, July 1990.

[166] A. E. Ennos, "Stresses developed in optical film coatings," Applied Optics, vol. 5, pp. 51-61, January 1966.

[167] M. Zecchino and T. Cunningham, Thin Film Stress Measurement Using Dektak Stylus Profilers. Veeco Instruments Inc., 2010. 